\title{
Trigonometry of spacetimes: a new self-dual approach to a curvature/signature (in)dependent trigonometry
}

\author{
Francisco J. Herranz ${ }^{\dagger}$, Ramón Ortega ${ }^{\star}$, and Mariano Santander ${ }^{\star}$ \\ † Departamento de Física, Escuela Politécnica Superior \\ Universidad de Burgos, E-09006 Burgos, Spain \\ * Departamento de Física Teórica, Facultad de Ciencias \\ Universidad de Valladolid, E-47011 Valladolid, Spain
}

\begin{abstract}
A new method to obtain trigonometry for the real spaces of constant curvature and metric of any (even degenerate) signature is presented. The method could be described as 'curvature/signature (in)dependent trigonometry' and encapsulates trigonometry for all these spaces into a single basic trigonometric group equation. This brings to its logical end the idea of an 'absolute trigonometry', and provides equations which hold true for the nine two-dimensional spaces of constant curvature and any signature. This family of spaces includes both relativistic and non-relativistic homogeneous spacetimes; therefore a complete discussion of trigonometry in the six de Sitter, minkowskian, Newton-Hooke and galilean spacetimes follow as particular instances of the general approach.

Distinctive traits of the method are 'universality' and '(self-)duality': every equation is meaningful for the nine spaces at once, and displays explicitly invariance under a duality transformation relating the nine spaces amongst themselves. These basic structural properties allow a complete study of trigonometry and in fact any equation previously known for the three classical (riemannian) spaces also has a version for the remaining six 'spacetimes'; in most cases these equations are new. The derivation of the single basic trigonometric equation at group level, its translation to a set of equations (cosine, sine and dual cosine laws) and the natural apparition of angular and lateral excesses, area and coarea are explicitly discussed in detail.

The exposition also aims to introduce the main ideas of this direct group theoretical way to trigonometry; this can be successfully applied for other rank-one spaces as well (e.g. the complex type, as the quantum space of states), and may well provide a path to systematically study trigonometry for any homogeneous symmetric space.
\end{abstract}




\section{Contents}

1. Introduction 3

2. Cayley-Klein geometries and spaces of real type 6

2.1. Cayley-Klein geometries in dimension $N \quad 6$

2.2. The nine two-dimensional real Cayley-Klein geometries $\quad 7$

2.3. Spacetimes as Cayley-Klein spaces 11

2.4. Realization of the spaces of points 12

3. The compatibility conditions for a triangular loop 13

$\begin{array}{ll}\text { 3.1. Loop excesses and loop equations } & 17\end{array}$

3.2. The basic trigonometric identity 19

4. The basic equations of trigonometry in the nine Cayley-Klein spaces 21

4.1. Alternative forms for the cosine theorems 23

4.2. Dependence and sets of basic equations 24

4.3. Relation with the usual approach and with absolute trigonometry 26

4.4. A compact notation 29

4.5. Area and coarea and the dualities length/area and angle/coarea 30

4.6. The trigonometric equations in the minimal form 32

5. A trigonometric bestiarium 32

5.1. Equations of Euler, Gauss-Delambre-Mollweide and Napier 33

5.2. Equations for area and coarea $\quad 35$

5.3. Some historical comments 37

5.4. Existence conditions 38

6. Other types of triangles 39

6.1. Second-kind triangles 39

6.2. Orthogonal triangles 39

7. On the trigonometry of homogeneous spacetimes 43

8. Concluding remarks 46

Acknowledgments $\quad 47$

Appendix: Some relations for the trigonometric functions $\quad 47$

References $\quad 49$ 


\section{Introduction}

Trigonometry of relativistic homogeneous, constant curvature models of spacetimes (antide Sitter, Minkowski and de Sitter) is the most elementary part of the geometry in these spacetimes. However, it has yet to become part of common mathematical or theoretical physicist knowledge. Trigonometry in minkowskian spacetime was first explicitly studied by Birman and Nomizu [1] and except for some results in Yaglom's book [2] —under the names of cohyperbolic and doubly hyperbolic geometries - we have not found any explicit formulation for the trigonometry in either anti-de Sitter or de Sitter spacetimes, in spite of the very basic nature and strong current interest in these spaces. Thus a first and short term aim of this paper is to fill in this gap.

There is also a second, more long term aim. Trigonometry, the study of the simplest geometrical configuration in a given space, should be a basic building block within the specific study of the geometry of homogeneous symmetric spaces. Hence this paper should also (and in the long run, mainly) be read as a step within the general programme of studying trigonometry of symmetric spaces (see [3]-[6]).

Within this perspective, the final and primary aim of this work is to introduce the ideas and methods of a group theoretical derivation to trigonometry which we believe to be new. This approach does not consider trigonometry for a single space (for, say, the anti-de Sitter spacetime), but instead is addressed to providing simultaneously the trigonometry of a whole family of spaces. This approach carries to its logical end the 'absolute trigonometry' — first discussed by Bolyai and then continued by de Tilly amongst others [7, 8] — which covered simultaneously the three classical spaces of constant curvature (sphere, euclidean space and Lobachewski hyperbolic space). This 'absolute trigonometry' can be considered as a first, albeit partial step in the direction we are pursuing here.

There are several distinctive traits in this approach. First, economy of thought: a single (parameter dependent) computation covers at once the trigonometry of several spaces. Second, a clear view is obtained for relationships between different spaces in the same family, such as several dualities; otherwise some of these may easily pass unnoticed, yet they may provide new hindsights. Third, limiting (contracted) cases, corresponding to vanishing curvature and/or degenerate metric, are included and described at the same level as the generic ones, thus making completely redundant a separate study of contractions. These traits apply not only for trigonometry, but also for the study of most properties of geometries, groups and algebras within each family [9]-[17]; for instance superintegrable potentials with constants of motion quadratic in the momenta can be studied simultaneously for the sphere, the euclidean plane and the hyperbolic plane [18].

All symmetric homogeneous spaces can be classed into several natural families [13, 19, 20], each with their Lie groups of motion, Lie algebras, etc., which depend on some parameters distinguishing family members. In the simplest cases (the spaces of real type, associated to the quasi-orthogonal Lie groups obtained by contraction of $S O(N)$ and $S O(p, q))$ these parameters determine the curvatures and/or the signatures of the fundamental metric for each space in the family. Additional parameters in other families label a division algebra $(\mathbb{C}, \mathbb{H}, \mathbb{O})$ or a pseudo-division variant coordinatising the space.

The method we are proposing should furnish trigonometry for all these families of spaces. So far this goal has been completely accomplished for all rank-one homogeneous symmetric spaces, of real type as well as of complex, quaternionic or exceptional octonionic 
(Cayley) type. As it seems impossible to squeeze all this material - without seriously impairing the possibility of a clear exposition - for both the real and the complex spaces into a single paper, we feel it justified to devote a first paper to the introduction of the method and the discussion of the trigonometry of the nine two-dimensional spaces of real type. This will also serve as a background to underlie a follow up forthcoming paper [21] devoted to the trigonometry of complex spaces. Therefore in this paper we restrict to a complete and detailed discussion of trigonometry of the rank-one symmetric homogeneous spaces of real type, also called quadratic or orthogonal Cayley-Klein spaces (see e.g. $[12,15])$. Any three points in any rank-one real type homogeneous symmetric space are always contained in a two-dimensional (2D) totally geodesic submanifold, so considering only 2D spaces (planes) is no restriction at all.

There are $3^{N}$ real Cayley-Klein spaces in dimension $N$, thus nine $2 \mathrm{D}$ real quadratic Cayley-Klein spaces [2]: the sphere, euclidean and hyperbolic planes, the co-euclidean, galilean and co-minkowskian planes and finally the co-hyperbolic, minkowskian and doubly hyperbolic planes. Out of this list only the three spaces mentioned in the first place belong to the restricted family of the so-called two-point homogeneous spaces whose trigonometry is very well known. The remaining six spaces are not two-point homogeneous according to the usual definition [22] (because the action of the isotropy group on the tangent space 'unit sphere' is not transitive), but together with the three previous ones they provide a natural frame for a joint study of trigonometry. Within a concrete physical interpretation these six spaces are the $(1+1) \mathrm{D}$ symmetric homogeneous spacetimes: oscillating (or anti) Newton-Hooke, galilean, expanding Newton-Hooke $(1+1) D$ spacetimes, and antide Sitter, minkowskian and de Sitter $(1+1) \mathrm{D}$ spacetimes. The trigonometry of the two constant curvature counterparts of the special relativity spacetime, mentioned as a short term first aim of this work, follows as a sideline byproduct. The required information on Cayley-Klein spaces of real type, in particular on the nine 2D spaces, is given in Section 2 .

The method we propose is presented in Section 3. It embodies the trigonometry for the whole biparametric family of these real 2D Cayley-Klein spaces into a single group equation for the parameter-dependent group of motions of the corresponding space. Dealing with a whole family at once, this equation, that we call the basic trigonometric identity, gives a perspective on some relationships between different spaces which goes beyond the treatments devoted to the study of a single space; this is so because these relationships involve simultaneously several (and at least two) different spaces. The simplest such relations are the contractions (e.g. from anti-de Sitter or de Sitter trigonometries to the minskowskian one), whose description is built-in automatically in the Cayley-Klein scheme. However, there are others, such as a fundamental duality (or polarity) and an interesting triality. Duality is the main structural backbone in our approach, and the requirement to explicitly maintaining duality in all expressions and at all stages acts as a kind of method 'fingerprint'. This duality should not be confused with the ordinary Cartan duality for pairs of symmetric spaces [23] which also has a very natural description in our approach.

The basic trigonometric equation is also directly related to other product formulas, which embody the Gauss-Bonnet theorem for triangles as well as its dual theorem; the proof for these equations is also given as they appear as an integral part in the derivation of the basic trigonometric identity. Each of these formulas contains trigonometry in a nutshell, but to unveil explicitly the trigonometric equations, it is better to start from the basic trigonometric identity. This procedure allows a very rapid browsing through 
the complete $z o o$ of trigonometric equations for the nine spaces with an effort definitely lower than that required for studying any single case, because duality acts as a kind of 'superstructural' requirement. All the equations we obtain for any of the nine 2D spaces in the real Cayley-Klein family are very well known in the three constant curvature riemannian cases (spherical, euclidean and hyperbolic) but we have not found any reference to the anti-de Sitter and de Sitter versions of most of these formulas, especially those involving areas and coareas of triangular loops. All this material is covered in Sections 4 and 5, and includes a complete bestiarium of equations valid in all the nine spaces, as well as some brief historical comments to relate the general equations to their spherical or euclidean counterparts (which have been known for centuries). A length/area duality recently pointed out by Arnol'd [24] also follows naturally from our approach, suggesting the extension of the spherical triality introduced there to a triality between the hyperbolic plane, the anti-de Sitter spacetime and the de Sitter spacetime which would be worth studying.

In the generic 2D Cayley-Klein space there may be two types of lines (time- and spacelike in the kinematical interpretation). The main body of the paper deals with triangles whose sides are of a single particular type, but a brief report of the results obtained for the remaining types of triangles is given in Section 6; these include triangles with two orthogonal sides. In Section 7 we translate some of the results to the kinematical language, and offer several trigonometric relations for the de Sitter and anti-de Sitter spacetimes, as well as for their non-relativistic analogous.

There is a small price to pay for this wide scope: the use of a non-standard notation in which the circular, hyperbolic and their common intermediate parabolic functions appear altogether under the guise of 'labeled' trigonometric functions, which reduce to the two familiar cases for the label values $\kappa=1,-1$ (see e.g. [9, 11]). The main part of the paper is couched using these functions, but to help the reader get a quick appraisal, we have included several tables in which a sample of the results are displayed in the conventional notation; these examples may help to translate any of the equations we give either to their 'natural' form, where the non-zero labels are reduced to their standard $1,-1$ values by using 'natural' units as in table 2, or to a form involving explicitly the constants determining the curvature and signature of each space, as the universe (time) radius $\tau$ and the relativistic constant $c$ in table 4. A Section with some final comments and prospects for continuation of this work closes the paper.

Although it is not our aim to cover here any applications, we should point to the relevance of many of the complicated trigonometric equations whose 'general' form we derive in several fields. For instance they appear in the Zamolodchikov solution for tetrahedral equations as factorization condition for the $S$-matrix in $(1+2) \mathrm{D}[25,26]$, both reproduced in [27]. The extension of the Moyal type formulation of Quantum Mechanics to spaces with constant curvature also involves many of the complicated equations for spherical or hyperbolic area in terms of sides. Trigonometry in the relativistic de Sitter spacetimes can be also used to get understanding of their global structure, the presence of horizons, etc., just as hyperbolic trigonometry is essential for a detailed understanding of the hyperbolic plane. 


\section{Cayley-Klein geometries and spaces of real type}

\subsection{Cayley-Klein geometries in dimension $N$}

We only give a brief summary of the $N$-dimensional Cayley-Klein (herafter CK) geometries, enough to allow a meaningful discussion of trigonometry in $(1+3) \mathrm{D}$ spacetimes. For more specific details, the reader can consult $[12,15,16]$.

The real Lie algebra $\mathfrak{s o}(N+1)$ has a $\mathbb{Z}_{2}^{\otimes N}$ group of commuting involutive automorphisms determining a grading. A particular subfamily of all graded contractions of $\mathfrak{s o}(N+1)$ depend on $N$ real parameters $\kappa_{1}, \kappa_{2}, \ldots, \kappa_{N}$ and are called orthogonal CK algebras, since they are exactly the motion algebras of the geometries of a real space with a projective metric in the CK sense $[28,29]$. In this theory, the pencil of points in a line can be either elliptic/parabolic/hyperbolic, the pencil of lines through a point in a 2-plane can be also elliptic/parabolic/hyperbolic, the same happens for the pencil of 2-planes through a line in a 3-plane, etc. Each alternative is described by one of the real constants, $\kappa_{1}, \kappa_{2}, \kappa_{3}, \ldots$, and the elliptic/parabolic/hyperbolic character corresponds in each case to $\kappa_{1}, \kappa_{2}, \kappa_{3}, \ldots$ being $>0 /=0 /<0$.

These algebras depending on $N$ real coefficients are denoted as $\mathfrak{s o}_{\kappa_{1}, \kappa_{2}, \ldots, \kappa_{N}}(N+1)$. Their $(N+1) N / 2$ generators are $P_{i}, J_{i j}, i, j=1, \ldots N, i<j$, and have a vector representation by $(N+1) \times(N+1)$ real matrices:

$$
P_{i}=-\kappa_{0 i} e_{0 i}+e_{i 0} \quad J_{i j}=-\kappa_{i j} e_{i j}+e_{j i}
$$

where the $\kappa$ with two indices are defined as $\kappa_{a b}:=\kappa_{a+1} \kappa_{a+2} \ldots \kappa_{b}$ and $e_{a b}$ is the matrix with a single non-zero entry, 1 , in the row $a$ and column $b, a, b=0,1, \ldots, N$. These expressions suggest a close association $P_{i} \leftrightarrow \kappa_{0 i}=\kappa_{1} \kappa_{2} \ldots \kappa_{i}$ and $J_{i j} \leftrightarrow \kappa_{i j}=\kappa_{i} \kappa_{i+1} \ldots \kappa_{j}$; the $\kappa$ associated to each generator will be called its label.

By matrix exponentiation this matrix realization generates a group of matrices of order $N+1$ denoted $S O_{\kappa_{1}, \kappa_{2}, \ldots, \kappa_{N}}(N+1)$. When all constants are different from zero the group $S O_{\kappa_{1}, \kappa_{2}, \ldots, \kappa_{N}}(N+1)$ is a simple group isomorphic to a (pseudo) orthogonal group $S O(p, q)$ with $(p, q)$ being the number of (positive, negative) terms in the sequence $\left(1, \kappa_{1}, \kappa_{1} \kappa_{2}, \kappa_{1} \kappa_{2} \kappa_{3}, \ldots, \kappa_{1} \kappa_{2} \cdots \kappa_{N}\right)$. When some constants are equal to zero $S O_{\kappa_{1}, \kappa_{2}, \ldots, \kappa_{N}}(N+1)$ is a non-simple contraction of $S O(p, q)$; this group is near enough to a simple group to warrant the name quasi-simple. By suitable scale changes in the generators, all constants $\kappa_{i}$ can be reduced to their 'canonical' values $1,0,-1$. The notation has been chosen so that when all constants $\kappa_{i}$ are equal to 1 , the group $S O_{\kappa_{1}, \kappa_{2}, \ldots, \kappa_{N}}(N+1)$ reduces to $S O(N+1)$.

Each constant $\kappa_{i}\left(\right.$ or $\left.\kappa_{a b}\right)$ is linked to an involutive automorphism of the Lie algebra, so a symmetric homogeneous space can be obtained as the coset space $S O_{\kappa_{1}, \kappa_{2}, \ldots, \kappa_{N}}(N+$ 1) $/ H_{(i)}$ where the subgroup $H_{(i)}$ is generated by the elements in the Lie algebra which are invariant under the involution associated to the constant $\kappa_{i}$. In particular, the involution associated to $\kappa_{1}$ has as the subgroup $H_{(1)}$ the CK group $S O_{\kappa_{2}, \ldots, \kappa_{N}}(N)$ generated by the $J_{i j}, i, j=1, \ldots N, i<j$.

The more relevant point to stress is the double role the constants $\kappa_{i}$ play. From the projective viewpoint they determine the nature of the pencil of points in a line, of a pencil of lines in a 2-plane, etc. From the metric viewpoint these constants determine the curvature and the signature of the quadratic metric naturally induced in each of the spaces 
$S O_{\kappa_{1}, \kappa_{2}, \ldots, \kappa_{N}}(N+1) / H_{(i)}$ by the 'Killing-Cartan' metric in the group (see [16] for details). Specifically, $\kappa_{i}$ is the curvature ('constant' in a suitable sense) of this homogeneous space, and the remaining $\kappa$ constants determine the signature of the metric. For the particular homogeneous space $S O_{\kappa_{1}, \kappa_{2}, \ldots, \kappa_{N}}(N+1) / S O_{\kappa_{2}, \ldots, \kappa_{N}}(N)$ the curvature is constant in the usual sense and equals to $\kappa_{1}$, while the signature of the metric is given by the diagonal matrix with entries $\left(1, \kappa_{2}, \kappa_{2} \kappa_{3}, \ldots, \kappa_{2} \cdots \kappa_{N}\right)$.

Let us comment on some of the spaces in the family $S O_{\kappa_{1}, \kappa_{2}, \ldots, \kappa_{N}}(N+1) / S O_{\kappa_{2}, \ldots, \kappa_{N}}(N)$. When all constants are equal to 1 , this space is the standard ND sphere $S^{N}$ of curvature 1 ; the values $\left\{\kappa_{1}, \kappa_{2}, \ldots, \kappa_{N}\right\}=\{\kappa>0,1, \ldots, 1\}$ lead to the $N D$ sphere of (positive) curvature $\kappa$. Other choices are $\{\kappa<0,1, \ldots, 1\}$, leading to the ND Lobachewski space $H^{N}$ of (negative) curvature $\kappa$, or $\{0,1, \ldots 1\}$ which leads to the flat $N D$ euclidean space $E^{N}$. Thus the three classical homogeneous spaces of constant curvature and definite positive metric are all included in the CK family. But there are many others. For instance, the $(1+3) \mathrm{D}$ Minkowski spacetime of special relativity corresponds to $\{0,-1,1,1\}$ : it is a flat space with a metric of $(1,3)$ type. The projective interpretation also agrees with the choice of $\kappa_{i}$ for Minkowski spacetime: the pencil of events in a time-like line is parabolic (separation measured by proper time, $\kappa_{1}=0$ ), the pencil of time-like lines through an event in a time-like 2-plane is hyperbolic (separation measured by the rapidity, $\kappa_{2}<0$ ), while the pencils of time-like 2-planes in a time-like 3-plane and of time-like 3-planes in the $(1+3) \mathrm{D}$ spacetime are clearly elliptic (separation measured by plane and dihedral space angles, $\kappa_{3}>0, \kappa_{4}>0$ ). The anti-de Sitter spacetime has $\kappa_{1}>0$ (that is, the pencil of events on a time-like line is elliptic), and the same remaining constants as the minkowskian one, etc.

The CK algebras, groups and spaces have many subgroups, subalgebras and subspaces of the same CK type. Generically, any translation in the space $S O_{\kappa_{1}, \kappa_{2}, \ldots, \kappa_{N}}(N+$ 1)/SO $\mathrm{\kappa}_{2}, \ldots, \kappa_{N}(N)$ is conjugated to (at least) one of the $N$ basic translations $P_{1}, P_{2}, \ldots, P_{N}$. Three points in such a space will determine three translations, which will translate along lines contained in a 2-plane, and the rotation generator in this plane will be also generically conjugated to (at least) one of the $N(N-1) / 2$ rotation generators $J_{i j}$. Generically and up to a group motion, these three points can always be assumed to lie on the 2-plane generated by say $P_{m}, P_{n}, m<n$; these generators together with the rotation generator $J_{m n}$ close a CK subalgebra $\mathfrak{s o}_{\kappa_{0 m}, \kappa_{m n}}(3)$. Thus consideration of the $2 \mathrm{D}$ case will suffice for a complete study of trigonometry, and therefore in the rest of the paper we will only discuss the $2 \mathrm{D}$ spaces, described by two constants $\kappa_{1}, \kappa_{2}$. When considering trigonometry in spaces of higher dimension, the two constants should be understood in the light of these remarks.

\subsection{The nine two-dimensional real Cayley-Klein geometries}

The motion groups of the nine 2D CK geometries of real type can be described in a unified setting by means of two real coefficients $\kappa_{1}, \kappa_{2}$ and are collectively denoted $S O_{\kappa_{1}, \kappa_{2}}(3)$. The generators $\left\{P_{1}, P_{2}, J_{12}\right\}$ of the corresponding Lie algebras $\mathfrak{s o}_{\kappa_{1}, \kappa_{2}}(3)$ have Lie commutators:

$$
\left[J_{12}, P_{1}\right]=P_{2} \quad\left[J_{12}, P_{2}\right]=-\kappa_{2} P_{1} \quad\left[P_{1}, P_{2}\right]=\kappa_{1} J_{12} .
$$

There is a single Lie algebra Casimir coming from the Killing-Cartan form:

$$
\mathcal{C}=P_{2}^{2}+\kappa_{2} P_{1}^{2}+\kappa_{1} J_{12}^{2} .
$$


The CK algebras in the quasi-orthogonal family $\mathfrak{s o}_{\kappa_{1}, \kappa_{2}}(3)$ can be endowed with a $\mathbb{Z}_{2} \otimes \mathbb{Z}_{2}$ group of commuting automorphisms generated by:

$$
\begin{aligned}
& \Pi_{(1)}:\left(P_{1}, P_{2}, J_{12}\right) \rightarrow\left(-P_{1},-P_{2}, J_{12}\right) \\
& \Pi_{(2)}:\left(P_{1}, P_{2}, J_{12}\right) \rightarrow\left(P_{1},-P_{2},-J_{12}\right) .
\end{aligned}
$$

The two remaining involutions are the composition $\Pi_{(02)}=\Pi_{(1)} \cdot \Pi_{(2)}$ and the identity. Each involution $\Pi$ determines a subalgebra of $\mathfrak{s o}_{\kappa_{1}, \kappa_{2}}(3)$, denoted $\mathfrak{h}$, whose elements are invariant under $\Pi$; the subgroups generated by these subalgebras will be denoted by $H$ with the same subindices as the involution itself.

The elements defining a 2D CK geometry are as follows $[9,10]$ :

- The plane as the set of points corresponds to the 2D symmetrical homogeneous space

$$
S_{\left[\kappa_{1}\right], \kappa_{2}}^{2} \equiv S O_{\kappa_{1}, \kappa_{2}}(3) / H_{(1)} \equiv S O_{\kappa_{1}, \kappa_{2}}(3) / S O_{\kappa_{2}}(2) \quad H_{(1)}=\left\langle J_{12}\right\rangle \approx S O_{\kappa_{2}}(2) .
$$

The generator $J_{12}$ leaves a point $O$ (the origin) invariant, thus $J_{12}$ acts as the rotation around $O$. The involution $\Pi_{(1)}$ is the reflection around the origin. In this space $P_{1}$ and $P_{2}$ generate translations which move the origin point in two basic directions.

- The set of lines is identified as the 2D symmetrical homogeneous space

$$
S_{\kappa_{1},\left[\kappa_{2}\right]}^{2} \equiv S O_{\kappa_{1}, \kappa_{2}}(3) / H_{(2)} \equiv S O_{\kappa_{1}, \kappa_{2}}(3) / S O_{\kappa_{1}}(2) \quad H_{(2)}=\left\langle P_{1}\right\rangle \approx S O_{\kappa_{1}}(2) .
$$

In this space, the generator $P_{1}$ leaves invariant the 'origin' line $l_{1}$, which is moved in two basic directions by $J_{12}$ and $P_{2}$. Therefore, within $S_{\kappa_{1},\left[\kappa_{2}\right]}^{2}, P_{1}$ should be interpreted as the generator of 'rotations' around $l_{1}$, and the involution $\prod_{(2)}$ is the reflexion in $l_{1}$.

- There is a second set of lines corresponding to the 2D symmetrical homogeneous space

$$
S O_{\kappa_{1}, \kappa_{2}}(3) / H_{(02)} \equiv S O_{\kappa_{1}, \kappa_{2}}(3) / S O_{\kappa_{1} \kappa_{2}}(2) \quad H_{(02)}=\left\langle P_{2}\right\rangle \approx S O_{\kappa_{1} \kappa_{2}}(2) .
$$

In this case, it is the generator $P_{2}$ that leaves invariant an 'origin' line $l_{2}$ (considered as the elementary 'point') in this space while $J_{12}$ and $P_{1}$ do move $l_{2}$. The involution $\Pi_{(02)}$ is the reflexion in the line $l_{2}$.

In order to distinguish the two sets of lines we call the elements of $S_{\kappa_{1},\left[\kappa_{2}\right]}^{2}$ lines of first-kind while the elements of the space $S O_{\kappa_{1}, \kappa_{2}}(3) / H_{(02)}$ will be called lines of secondkind. By a two-dimensional CK geometry we will understand the set of three symmetrical homogeneous spaces of points, lines of first-kind and lines of second-kind. The group $S O_{\kappa_{1}, \kappa_{2}}(3)$ acts transitively on each of these spaces.

All properties of the two spaces of lines can be transcribed in terms of the space $S_{\left[\kappa_{1}\right], \kappa_{2}}^{2}$ itself, and in this interpretation the lines of first- or second-kind can be seen as certain $1 \mathrm{D}$ submanifolds of $S_{\left[\kappa_{1}\right], \kappa_{2}}^{2}$ rather than 'points' in the spaces $S_{\kappa_{1},\left[\kappa_{2}\right]}^{2}$ or $S O_{\kappa_{1}, \kappa_{2}}(3) / H_{(02)}$. In the following we will interpret everything in terms of the space $S_{\left[\kappa_{1}\right], \kappa_{2}}^{2}$, where $l_{1}$ and $l_{2}$ should be considered as two 'orthogonal' lines meeting in $O$. The notation has been chosen to implicitly suggest this, with $P_{1}$ translating along the line $l_{1}$ (resp. $P_{2}$ along $l_{2}$ ) as shown in figure 1.

The coefficients $\kappa_{1}, \kappa_{2}$ play a twofold role. The space $S_{\left[\kappa_{1}\right], \kappa_{2}}^{2}$ has a quadratic metric coming from the Casimir (2.3), whose signature corresponds to the diagonal matrix $\operatorname{diag}\left(1, \kappa_{2}\right)$. This metric is riemannian (definite positive) for $\kappa_{2}>0$, lorentzian (indefinite) 


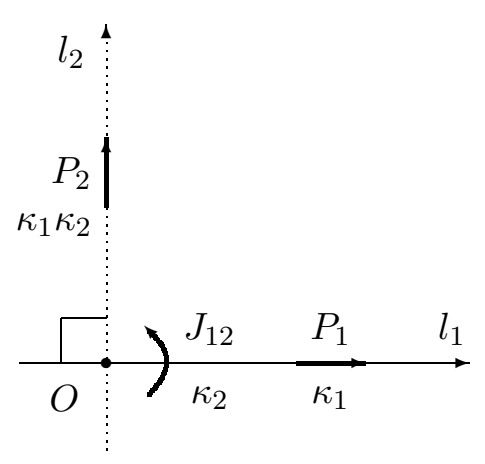

Figure 1: Generators and their associated labels in a 2D CK geometry.

for $\kappa_{2}<0$ and degenerate for $\kappa_{2}=0$. Next, the same space has a canonical conexion (as any symmetric space [30]) which is compatible with the metric, and has constant curvature equal to $\kappa_{1}$; the notation $S_{\left[\kappa_{1}\right], \kappa_{2}}^{2}$ for the space is intended to recall the analogy with the sphere $S^{2}$ to which $S_{\left[\kappa_{1}\right], \kappa_{2}}^{2}$ reduces when all the $\kappa_{i}$ constants are equal to 1 . The list of $\kappa_{i}$ values is appended to the symbol for the space; the $\kappa_{i}$ in square brackets is the constant curvature, and the remaining constant determines the signature. Alternatively, the coefficients $\kappa_{1}, \kappa_{2}$ determine the kind of measures of separation amongst points and lines in the Klein sense [2, 9]:

- The pencil of points on a first-kind line is elliptical/parabolical/hyperbolical according to whether $\kappa_{1}$ is greater than/equal to/lesser than zero.

- Likewise for the pencil of points on a second-kind line depending on the product $\kappa_{1} \kappa_{2}$.

- Likewise for the pencil of lines through a point according to $\kappa_{2}$.

For $\kappa_{1}$ positive/zero/negative the isotropy subgroup $H_{(2)}$ is $S O(2) / \mathbb{R} / S O(1,1)$, and the same happens for $H_{(1)}$ (resp. $\left.H_{(02)}\right)$ according to the value of $\kappa_{2}$ (resp. $\left.\kappa_{1} \kappa_{2} \equiv \kappa_{02}\right)$. Any of these three subgroups would lead to the 1D CK geometries with Lie algebra $\mathfrak{s o}_{\kappa}(2)$ and trivial isotropy subalgebra, which can be identified as the circunference $(S O(2)$ for $\kappa>0)$, the real line $(\mathbb{R} \equiv I S O(1)$ for $\kappa=0)$ and the hyperbolic line $(S O(1,1)$ for $\kappa<0)$.

Whenever the coefficient $\kappa_{1}$ (resp. $\kappa_{2}$ ) is different from zero, a suitable choice of length unit (resp. angle unit) allows us to reduce it to either +1 or -1 . By taking into account the possible values for $\left(\kappa_{1}, \kappa_{2}\right)$, we obtain nine $2 \mathrm{D}$ real CK geometries, whose groups of motion, commutation rules (2.2) and Casimirs (2.3) are written explicitly in table 1 together with the isotropy subgroups $H_{(1)}, H_{(2)}$ and $H_{(02)}$ of their associated symmetric spaces.

The same Lie group (up to isomorphism) can be the group of motion of more than one CK geometry. For instance, the euclidean group $I S O(2)$ is the group of motion of two CK geometries, characterized by two different sets for the three associated symmetric spaces. The space of points is $I S O(2) / S O(2)$ for euclidean geometry, but $I S O(2) / \mathbb{R}$ for the co-euclidean one; this last space can be identified as the space of (first-kind) lines in the euclidean plane. The same happens with $I S O(1,1)$. The simple group $S O(2,1)$ is associated to three CK geometries, namely hyperbolic, co-hyperbolic and doubly hyperbolic.

A fundamental property of the scheme of CK geometries is the existence of an 'automorphism' of the whole family, called ordinary duality $\mathcal{D}$. This is well defined for any 
Table 1: The nine two-dimensional CK geometries.

\begin{tabular}{|c|c|c|c|}
\hline \multirow{3}{*}{$\begin{array}{l}\text { Measure } \\
\text { of angle }\end{array}$} & \multicolumn{3}{|c|}{ Measure of distance } \\
\hline & Elliptic & Parabolic & Hyperbolic \\
\hline & $\kappa_{1}=1$ & $\kappa_{1}=0$ & $\kappa_{1}=-1$ \\
\hline \multirow{9}{*}{$\begin{array}{l}\text { Elliptic } \\
\kappa_{2}=1\end{array}$} & Elliptic & Euclidean & Hyperbolic \\
\hline & $S O(3)$ & $I S O(2)$ & $S O(2,1)$ \\
\hline & {$\left[J_{12}, P_{1}\right]=P_{2}$} & {$\left[J_{12}, P_{1}\right]=P_{2}$} & {$\left[J_{12}, P_{1}\right]=P_{2}$} \\
\hline & {$\left[J_{12}, P_{2}\right]=-P_{1}$} & {$\left[J_{12}, P_{2}\right]=-P_{1}$} & {$\left[J_{12}, P_{2}\right]=-P_{1}$} \\
\hline & {$\left[P_{1}, P_{2}\right]=J_{12}$} & {$\left[P_{1}, P_{2}\right]=0$} & {$\left[P_{1}, P_{2}\right]=-J_{12}$} \\
\hline & $\mathcal{C}=P_{2}^{2}+P_{1}^{2}+J_{12}^{2}$ & $\mathcal{C}=P_{2}^{2}+P_{1}^{2}$ & $\mathcal{C}=P_{2}^{2}+P_{1}^{2}-J_{12}^{2}$ \\
\hline & $H_{(1)}=S O(2)$ & $H_{(1)}=S O(2)$ & $H_{(1)}=S O(2)$ \\
\hline & $H_{(2)}=S O(2)$ & $H_{(2)}=\mathbb{R}$ & $H_{(2)}=S O(1,1)$ \\
\hline & $H_{(02)}=S O(2)$ & $H_{(02)}=\mathbb{R}$ & $H_{(02)}=S O(1,1)$ \\
\hline \multirow{10}{*}{$\begin{array}{l}\text { Parabolic } \\
\kappa_{2}=0\end{array}$} & Co-Euclidean & Galilean & Co-Minkowskian \\
\hline & Oscillating NH & & Expanding NH \\
\hline & $I S O(2)$ & $I I S O(1)$ & $\operatorname{ISO}(1,1)$ \\
\hline & {$\left[J_{12}, P_{1}\right]=P_{2}$} & {$\left[J_{12}, P_{1}\right]=P_{2}$} & {$\left[J_{12}, P_{1}\right]=P_{2}$} \\
\hline & {$\left[J_{12}, P_{2}\right]=0$} & {$\left[J_{12}, P_{2}\right]=0$} & {$\left[J_{12}, P_{2}\right]=0$} \\
\hline & {$\left[P_{1}, P_{2}\right]=J_{12}$} & {$\left[P_{1}, P_{2}\right]=0$} & {$\left[P_{1}, P_{2}\right]=-J_{12}$} \\
\hline & $\mathcal{C}=P_{2}^{2}+J_{12}^{2}$ & $\mathcal{C}=P_{2}^{2}$ & $\mathcal{C}=P_{2}^{2}-J_{12}^{2}$ \\
\hline & $H_{(1)}=\mathbb{R}$ & $H_{(1)}=\mathbb{R}$ & $H_{(1)}=\mathbb{R}$ \\
\hline & $H_{(2)}=S O(2)$ & $H_{(2)}=\mathbb{R}$ & $H_{(2)}=S O(1,1)$ \\
\hline & $H_{(02)}=\mathbb{R}$ & $H_{(02)}=\mathbb{R}$ & $H_{(02)}=\mathbb{R}$ \\
\hline \multirow{10}{*}{$\begin{array}{l}\text { Hyperbolic } \\
\kappa_{2}=-1\end{array}$} & Co-Hyperbolic & Minkowskian & Doubly Hyperbolic \\
\hline & Anti-de Sitter & & De Sitter \\
\hline & $S O(2,1)$ & $I S O(1,1)$ & $S O(2,1)$ \\
\hline & {$\left[J_{12}, P_{1}\right]=P_{2}$} & {$\left[J_{12}, P_{1}\right]=P_{2}$} & {$\left[J_{12}, P_{1}\right]=P_{2}$} \\
\hline & {$\left[J_{12}, P_{2}\right]=P_{1}$} & {$\left[J_{12}, P_{2}\right]=P_{1}$} & {$\left[J_{12}, P_{2}\right]=P_{1}$} \\
\hline & {$\left[P_{1}, P_{2}\right]=J_{12}$} & {$\left[P_{1}, P_{2}\right]=0$} & {$\left[P_{1}, P_{2}\right]=-J_{12}$} \\
\hline & $\mathcal{C}=P_{2}^{2}-P_{1}^{2}+J_{12}^{2}$ & $\mathcal{C}=P_{2}^{2}-P_{1}^{2}$ & $\mathcal{C}=P_{2}^{2}-P_{1}^{2}-J_{12}^{2}$ \\
\hline & $H_{(1)}=S O(1,1)$ & $H_{(1)}=S O(1,1)$ & $H_{(1)}=S O(1,1)$ \\
\hline & $H_{(2)}=S O(2)$ & $H_{(2)}=\mathbb{R}$ & $H_{(2)}=S O(1,1)$ \\
\hline & $H_{(02)}=S O(1,1)$ & $H_{(02)}=\mathbb{R}$ & $H_{(02)}=S O(2)$ \\
\hline
\end{tabular}

dimension, and in the $2 \mathrm{D}$ case it is given by:

$$
\mathcal{D}:\left(P_{1}, P_{2}, J_{12}\right) \rightarrow\left(-J_{12},-P_{2},-P_{1}\right) \quad \mathcal{D}:\left(\kappa_{1}, \kappa_{2}\right) \rightarrow\left(\kappa_{2}, \kappa_{1}\right) .
$$

The map $\mathcal{D}$ leaves the general commutation rules (2.2) invariant while it interchanges the space of points with the space of first-kind lines, $S_{\left[\kappa_{1}\right], \kappa_{2}}^{2} \leftrightarrow S_{\kappa_{1},\left[\kappa_{2}\right]}^{2}$, and the corresponding curvatures $\kappa_{1} \leftrightarrow \kappa_{2}$, preserving the space of second-kind lines. Note that $\mathcal{D}$ relates in general two different CK geometries, which are placed in symmetrical positions relative to the main diagonal in table 1. For instance, duality interchanges the euclidean, hyperbolic and minkowskian geometries with the co-euclidean, co-hyperbolic and co-minkowskian ones, while elliptic, galilean and doubly hyperbolic are self-dual geometries. This suggests also a kind of duality between curvature and signature which seems worth studying.

The non-generic situation where a coefficient $\kappa_{i}$ vanishes corresponds to an InönüWigner contraction [31]. The limit $\kappa_{1} \rightarrow 0$ is a local-contraction (around a point); it carries the geometries of the first and third columns of table 1 to the middle one, which have 'flat' spaces of points. The limit $\kappa_{2} \rightarrow 0$ is an axial-contraction (around a line), which carries the geometries of the first and third rows to the middle one. However, note that our approach to contractions is indeed built-in in the general expressions associated to the CK geometries and groups. This means that we deal with expressions that contain 
explicitly the constants $\kappa_{i}$ (which determine the curvature and signature of the space) and which can be also understood as contraction parameters so that a contraction is simply equivalent to putting $\kappa_{i}=0$ in the desired relation.

\subsection{Spacetimes as Cayley-Klein spaces}

It is rather remarkable that the meaningful kinematical spacetimes and their invariance groups [32] arise as particular CK spaces and groups. Let $\mathcal{H}, \mathcal{P}$ and $\mathcal{K}$ be the generators of time translations, space translations and boosts, respectively, in the most simple $(1+1) \mathrm{D}$ homogeneous spacetime. Under the identification

$$
P_{1} \equiv \mathcal{H} \quad P_{2} \equiv \mathcal{P} \quad J_{12} \equiv \mathcal{K}
$$

the six CK groups with $\kappa_{2} \leq 0$ (second and third rows of table 1; NH means NewtonHooke) are the motion groups of $(1+1) \mathrm{D}$ spacetimes. The physical reading of the three CK spaces within each of these CK geometries is:

- $S_{\left[\kappa_{1}\right], \kappa_{2}}^{2}$ is a $(1+1) \mathrm{D}$ spacetime, and points in $S_{\left[\kappa_{1}\right], \kappa_{2}}^{2}$ are spacetime events; the spacetime curvature equals $\kappa_{1}$ and is related to the usual universe radius $\tau$ (measured in time units) by $\kappa_{1}= \pm 1 / \tau^{2}$. Relativistic spacetimes occur for $\kappa_{2}<0$ (the signature of the minkowskian type metric is $\left.\operatorname{diag}\left(1,-1 / c^{2}\right)\right)$ and their non-relativistic limits correspond to $\kappa_{2}=0$.

- The space of first-kind lines $S_{\kappa_{1},\left[\kappa_{2}\right]}^{2}$ corresponds to the space of time-like lines. Here the coefficient $\kappa_{2}$ can be read as the curvature of the space of time-like lines, linked to the fundamental relativistic constant $c$ as $\kappa_{2}=-1 / c^{2}$. From this point of view, the passage from non-relativistic theories to relativistic ones can be looked as the introduction of a non-zero, negative, curvature in the space of time-like lines, which previously was flat.

- The space of second-kind lines $S O_{\kappa_{1}, \kappa_{2}}(3) / H_{(02)}$ is the $2 \mathrm{D}$ space of space-like lines.

According to the signs of the pair $\left(\kappa_{1}, \kappa_{2}\right)$ we have three homogeneous 'absolute-time' spacetimes for $\kappa_{2}=0$, namely, oscillating (or anti) Newton-Hooke for $\kappa_{1}>0$, galilean for $\kappa_{1}=0$ and expanding Newton-Hooke for $\kappa_{1}<0$; they are degenerate riemannian spacetimes with constant curvature $\kappa_{1}$ and a degenerate ('absolute-time') metric, which is the $c=\infty$ limit of the time metric in relativity. For $\kappa_{2}=-1 / c^{2}<0$ we find three 'relative-time' spacetimes: anti-de Sitter $\left(\kappa_{1}>0,-1 / c^{2}\right)$, minkowskian $\left(0,-1 / c^{2}\right)$ and de Sitter $\left(\kappa_{1}<0,-1 / c^{2}\right)$; these are pseudoriemmanian spacetimes with a metric of lorentzian type and constant curvature $\kappa_{1}$. Note that our choice of metric lets the curvature of anti-de Sitter be positive, which is opposite to the standard choice (where the metric is taken with the opposite sign) but fits to the closeness of time-like lines, which have been taken as the 'basic' ones; space-like lines are open in anti-de Sitter. The limits $\kappa_{1} \rightarrow 0 \equiv \tau \rightarrow \infty$ and $\kappa_{2} \rightarrow 0 \equiv c \rightarrow \infty$ correspond to a spacetime contraction and a speed-space contraction, respectively.

The three remaining geometries with $\kappa_{2}>0$ do not admit such a kinematical interpretation. They are the well known riemannian spaces with constant curvature $\kappa_{1}$. In these cases, the sets of first- and second-kind lines coincide, because in these cases (and only these) the generators $P_{1}$ and $P_{2}$ are conjugated under the full motion group:

$$
P_{2}=e^{\frac{\pi}{2 \sqrt{\kappa_{2}}} J_{12}} P_{1} e^{-\frac{\pi}{2 \sqrt{\kappa_{2}}} J_{12}} .
$$

This is why only these three spaces fulfil the usual definition of two-point homogeneity; 
as we show in this paper there is no compelling reason (and some drawbacks) to restrict any joint study only to these three cases.

\subsection{Realization of the spaces of points}

The following $3 \mathrm{D}$ real matrix representation of the CK algebra $\mathfrak{s o}_{\kappa_{1}, \kappa_{2}}(3)$ :

$$
P_{1}=\left(\begin{array}{ccc}
0 & -\kappa_{1} & 0 \\
1 & 0 & 0 \\
0 & 0 & 0
\end{array}\right) \quad P_{2}=\left(\begin{array}{ccc}
0 & 0 & -\kappa_{1} \kappa_{2} \\
0 & 0 & 0 \\
1 & 0 & 0
\end{array}\right) \quad J_{12}=\left(\begin{array}{ccc}
0 & 0 & 0 \\
0 & 0 & -\kappa_{2} \\
0 & 1 & 0
\end{array}\right)
$$

gives rise to a natural realization of the CK group $S O_{\kappa_{1}, \kappa_{2}}(3)$ as a group of linear transformations in an ambient linear space $\mathbb{R}^{3}=\left(x^{0}, x^{1}, x^{2}\right)$ in which $S O_{\kappa_{1}, \kappa_{2}}(3)$ acts as the group of linear isometries of a bilinear form with matrix:

$$
\Lambda=\left(\begin{array}{ccc}
1 & 0 & 0 \\
0 & \kappa_{1} & 0 \\
0 & 0 & \kappa_{1} \kappa_{2}
\end{array}\right)
$$

The exponential of the matrices (2.11) leads to a representation of the one-parametric subgroups $H_{(2)}, H_{(02)}$ and $H_{(1)}$ generated by $P_{1}, P_{2}$ and $J_{12}$ as:

$$
\begin{aligned}
\exp \left(\alpha P_{1}\right) & =\left(\begin{array}{ccc}
C_{\kappa_{1}}(\alpha) & -\kappa_{1} S_{\kappa_{1}}(\alpha) & 0 \\
S_{\kappa_{1}}(\alpha) & C_{\kappa_{1}}(\alpha) & 0 \\
0 & 0 & 1
\end{array}\right) \\
\exp \left(\beta P_{2}\right)= & \left(\begin{array}{ccc}
C_{\kappa_{1} \kappa_{2}}(\beta) & 0 & -\kappa_{1} \kappa_{2} S_{\kappa_{1} \kappa_{2}}(\beta) \\
0 & 1 & 0 \\
S_{\kappa_{1} \kappa_{2}}(\beta) & 0 & C_{\kappa_{1} \kappa_{2}}(\beta)
\end{array}\right) \\
\exp \left(\gamma J_{12}\right)= & \left(\begin{array}{ccc}
1 & 0 & 0 \\
0 & C_{\kappa_{2}}(\gamma) & -\kappa_{2} S_{\kappa_{2}}(\gamma) \\
0 & S_{\kappa_{2}}(\gamma) & C_{\kappa_{2}}(\gamma)
\end{array}\right)
\end{aligned}
$$

where the generalized cosine $C_{\kappa}(x)$ and sine $S_{\kappa}(x)$ functions are defined by [9]-[11]:

$$
\begin{array}{r}
C_{\kappa}(x):=\sum_{l=0}^{\infty}(-\kappa)^{l} \frac{x^{2 l}}{(2 l) !}= \begin{cases}\cos \sqrt{\kappa} x & \kappa>0 \\
1 & \kappa=0 \\
\cosh \sqrt{-\kappa} x & \kappa<0\end{cases} \\
S_{\kappa}(x):=\sum_{l=0}^{\infty}(-\kappa)^{l} \frac{x^{2 l+1}}{(2 l+1) !}=\left\{\begin{array}{ll}
\frac{1}{\sqrt{\kappa}} \sin \sqrt{\kappa} x & \kappa>0 \\
x & \kappa=0 \\
\frac{1}{\sqrt{-\kappa}} \sinh \sqrt{-\kappa} x & \kappa<0
\end{array} .\right.
\end{array}
$$

Two other useful curvature-dependent functions are the 'versed sine' $V_{\kappa}(x)$ (note that $V_{\kappa}(x)$ is well defined even if $\left.\kappa=0\right)$ and the tangent $T_{\kappa}(x)$ given by:

$$
V_{\kappa}(x):=\frac{1}{\kappa}\left(1-C_{\kappa}(x)\right) \quad T_{\kappa}(x):=\frac{S_{\kappa}(x)}{C_{\kappa}(x)} .
$$

These generalized trigonometric functions coincide with the usual elliptic and hyperbolic ones for $\kappa=1$ and $\kappa=-1$ respectively; the case $\kappa=0$ provides the parabolic or 
galilean functions: $C_{0}(x)=1, S_{0}(x)=x$ and $V_{0}(x)=x^{2} / 2$. This slightly non-standard notation is the price one should pay for the ability of describing all spaces at once, but the effort is worth it. Several identities for these functions, which are necessary for further development, are included in the Appendix.

A generic element $R \in S O_{\kappa_{1}, \kappa_{2}}(3)$ can be written as the product of the matrices (2.13) and satisfies

$$
R^{T} \Lambda R=\Lambda
$$

The action of $S O_{\kappa_{1}, \kappa_{2}}(3)$ on $\mathbb{R}^{3}$ is linear but not transitive, since it conserves the quadratic form $\left(x^{0}\right)^{2}+\kappa_{1}\left(x^{1}\right)^{2}+\kappa_{1} \kappa_{2}\left(x^{2}\right)^{2}$. The subgroup $H_{(1)}$, whose matrix representation is $\exp \left(\gamma J_{12}\right)(2.13)$, is the isotropy subgroup of the point $O \equiv(1,0,0)$, that is, the origin in the space $S_{\left[\kappa_{1}\right], \kappa_{2}}^{2}$. The action becomes transitive on the orbit in $\mathbb{R}^{3}$ of the point $O$, which is contained in the 'sphere' $\Sigma$ :

$$
\Sigma \equiv\left(x^{0}\right)^{2}+\kappa_{1}\left(x^{1}\right)^{2}+\kappa_{1} \kappa_{2}\left(x^{2}\right)^{2}=1 .
$$

This orbit can be identified with the space of points $S_{\left[\kappa_{1}\right], \kappa_{2}}^{2} \equiv S O_{\kappa_{1}, \kappa_{2}}(3) / S O_{\kappa_{2}}(2)$ of the CK geometry and the coordinates $\left(x^{0}, x^{1}, x^{2}\right)$ are the Weierstrass coordinates, while $\left(x^{1} / x^{0}, x^{2} / x^{0}\right)$ are the Beltrami coordinates. We remark that this scheme includes under a common description all the familiar embeddings (the so called vector models) of the sphere, hyperbolic plane, anti-de Sitter and de Sitter spaces in a linear 3D ambient space, with a flat metric of either euclidean or lorentzian type; the induced metric on the CK sphere $\Sigma$ should be defined as the quotient by $\kappa_{1}$ of the restriction of the flat ambient metric $d l^{2}=\left(d x^{0}\right)^{2}+\kappa_{1}\left(d x^{1}\right)^{2}+\kappa_{1} \kappa_{2}\left(d x^{2}\right)^{2}(2.12)$; this is always well defined because the restriction of the flat metric $d l^{2}$ to the CK sphere contains $\kappa_{1}$ as a factor [16].

The expressions for the metric in parallel $a, h$ and polar coordinates $r, \chi$ relative to the origin point $O$ and line $l_{1}$ are:

$$
d s^{2}=C_{\kappa_{1} \kappa_{2}}^{2}(h) d a^{2}+\kappa_{2} d h^{2} \quad d s^{2}=d r^{2}+\kappa_{2} S_{\kappa_{1}}^{2}(r) d \chi^{2}
$$

and the canonical conexion is given by the non-zero conexion coefficients (in parallel and polar coordinates)

$$
\begin{aligned}
& \Gamma_{a a}^{h}=\kappa_{1} S_{\kappa_{1} \kappa_{2}}(h) C_{\kappa_{1} \kappa_{2}}(h) \quad \Gamma_{a h}^{a}=-\kappa_{1} \kappa_{2} T_{\kappa_{1} \kappa_{2}}(h) \\
& \Gamma_{\chi \chi}^{r}=-\kappa_{2} S_{\kappa_{1}}(r) C_{\kappa_{1}}(r) \quad \Gamma_{r \chi}^{\chi}=1 / T_{\kappa_{1}}(r) \text {. }
\end{aligned}
$$

\section{The compatibility conditions for a triangular loop}

We now come to the main objective of this paper, which is the study of trigonometry of the nine CK spaces introduced in the previous Section.

In the euclidean plane three points always determine unambiguosly a triangle, because any two points are connected by a single geodesic segment, and all triangles are in an obvious sense of the same type. In the sphere three points do not directly determine a triangle, because two generic points can be joined by two geodesic segments (both lying on the same geodesic), yet all triangles are of the same type. In the minkowskian plane two points can always be joined by a single geodesic segment (like in the euclidean plane), but this segment can be of two generic and one non-generic types (time-like, space-like and isotropic) so here three points do determine unambiguously a triangle, but not all 
triangles are of the same type, and triangles with three time-like sides can coexist with triangles with mixed sides. Finally in the anti-de Sitter spacetime both complications may appear together: there are three types of sides, and two points with time-like separation can be joined, like in the sphere, by two different time-like geodesic segments.

To avoid unnecessary complications and such boring attention to details, it is better to introduce the concept of triangular loop, which affords a well defined replacement of the unprecise idea of 'triangle as three points'. A triangular loop can be considered either as a triangular point loop or as a triangular line loop, and we will need the simultaneous consideration of both aspects. Furthermore, and according to the type of the 'sides', there are several different types of triangular loops, which merge into a single type in the riemannian case $\kappa_{2}>0$.

From now on (except in Section 6), we will deal exclusively with first-kind triangular loops (i.e. time-like in the spaces with kinematical interpretation). A (first-kind) triangular point loop can be considered as two different (first-kind) paths for a point going from an initial position $C$ to a final one $B$. One path will be the direct one along the segment of the (first-kind) line $a$ determined by $C$ and $B$. The other will be a two-step path made of two segments of (first-kind) lines going from $C$ to an intermediary point $A$ along a line $b$ and then from $A$ to $B$ along the line $c$ (see figure 2a); hereafter we will omit the reference to the first-kind type of all lines. For most purposes it is better to look at the triangular point loop as a single (possibly open) poligonal curve (see figure 3), obtained from the line $a$ by replacing the segment $C B$ by the two geodesic segments $C A$ and $A B$; this curve will be considered as an oriented and cooriented curve, and will be only closed when the geodesic $a$ itself is closed. The corners in the poligonal may be smoothed (while maintaining the first-kind character) and thus we will obtain a smooth curve.

The previous view can be dualized, and the loop can be also considered as a triangular line loop: the dual of the single curve associated to the point loop is a moving line which starts at $a$, then rotates around $C$ going to $b$, then around $A$ towards $c$ and finally comes back to $a$ by means of a rotation around $B$ (see figure $2 \mathrm{~b}$ ). This can be also considered as an ordinary point loop in the dual space, because lines in the given space are interpreted as points in the dual space.

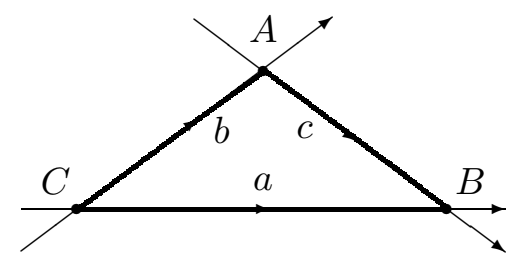

Figure 2: a) Triangular point loop.

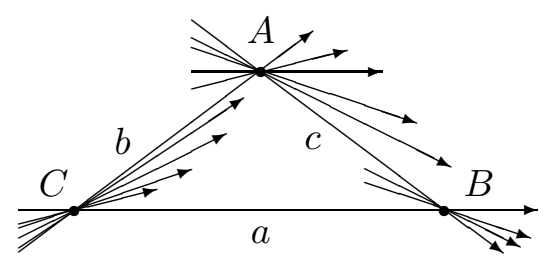

b) Triangular line loop.

In the kinematical interpretation of the six spacetimes with $\kappa_{2} \leq 0$, a triangular loop will therefore be determined by two time-like future pointing paths from an initial spacetime point $C$ to a final one $B$, one along the direct path and other through an intermediary spacetime point $A$. Note the assymmetric role the vertex $A$ plays from the beginning. Each side of the loop determines the generator of translations along the side, up to a non-zero scale factor which should be splitted into a sign (corresponding to one of the two possible 
orientations of the line) and a positive scale factor (corresponding to the choice of a unit length). The restriction to first-kind sides means that the following condition is satisfied:

P1) The three generators $P_{a}, P_{b}, P_{c}$ are either equal or opposite to some conjugate by means of some group transformation to the single fiducial generator of translations $P_{1}$ along first-kind lines.

We shall now perform a fiducial choice of the still undetermined factors in these generators, which will be assumed fixed from now on, according to a second condition:

P2) The positive sense of translation generated by $P_{a}, P_{b}, P_{c}$ agrees with the orientation for the point loop as a single curve.

The meaning of these conditions can be appreciated more clearly for the geometries with $\kappa_{2} \leq 0$, in the kinematical interpretation, for which the first condition embodies the time-like character of the three lines (here $P_{1} \equiv \mathcal{H}$ generates the future time translation along the fiducial time-like line), and the second condition corresponds to the future character of a time-like line loop. In the riemmanian cases $\left(\kappa_{2}>0\right)$ all geodesics can be considered to be simultaneously of both first- and second-kind; then first condition is automatic, while the second can always be clearly fulfilled.

Discussion of the trigonometry of triangular loops with sides of different kind can be developed in full analogy with the pure first-kind case discussed here; see Section 6 .

The important fact is that for any triangular loop, a choice of $P_{a}, P_{b}, P_{c}$ satisfying these two conditions is always possible, and this is so simultaneously for the nine CK geometries. Now we will denote $a$ the (positive, unoriented) distance between the points $C, B$ along the geodesic direct path (the geodesic segment on $a$ which is missing on the curve in figure 3 ), and by $b, c$ the (positive, unoriented) distances between the points $C, A$ and $A, B$ along the geodesic segments on the lines $b, c$ on the curve. The use of the same names for the lengths of the three loop sides and for the baselines themselves is traditional and should cause no confusion. In the kinematical cases, the lengths $a, b, c$ will be the proper times along the sides between their end events, and the angles $A, B, C$ are the relative rapidities between the time-like lines at each vertex (recall the rapidity is the natural angle in minkowskian geometry [33]). In kinematical spaces the triangle loop, seen as a single curve in figure 3 is the wordline of the travelling twin in the twin pseudoparadox.

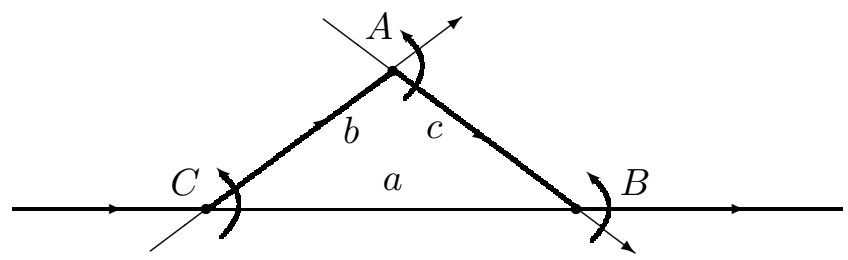

Figure 3: Triangular loop as a single curve.

On the dual hand, the generators $J_{A}, J_{B}, J_{C}$ of rotations around the vertices $A \equiv$ $b \cap c, B \equiv c \cap a, C \equiv a \cap b$ are again determined up to sign and a positive scale factor, which we shall choose so as to satisfy two conditions, dual to the previous ones:

J1) The three generators $J_{A}, J_{B}, J_{C}$ are conjugated by means of some group transformation to the single fiducial generator of rotations $J_{12}$. 
J2) The positive sense of rotation around each vertex is the correct one determined by the given orientation and coorientation of the loop as a curve.

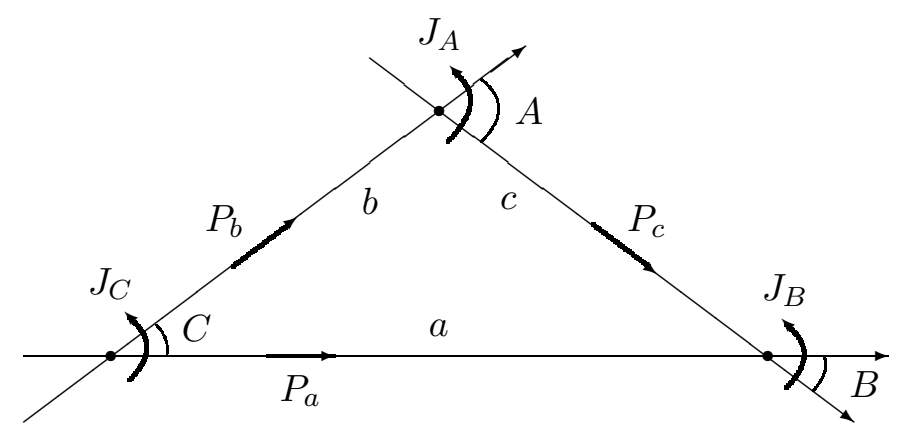

Figure 4: Pure first-kind triangle with three first-kind sides $a, b, c$, two inner angles $B, C$ and an external angle $A$, together with the generators of translations along the sides and the generators of rotations around the vertices.

The generators $P_{a}, P_{b}, P_{c} ; J_{A}, J_{B}, J_{C}$ are not independent. They are related by several compatibility conditions:

$$
\begin{array}{ll}
P_{b}=e^{C J_{C}} P_{a} e^{-C J_{C}} & J_{B}=e^{c P_{c}} J_{A} e^{-c P_{c}} \\
P_{c}=e^{-A J_{A}} P_{b} e^{A J_{A}} & J_{C}=e^{-a P_{a}} J_{B} e^{a P_{a}} \\
P_{a}=e^{B J_{B}} P_{c} e^{-B J_{B}} & J_{A}=e^{b P_{b}} J_{C} e^{-b P_{b}} .
\end{array}
$$

which can be considered as giving an implicit group theoretical definition for the three sides and the three angles; these definitions are in agreement with the ones derived from differential geometry with the metrics (2.19) in the spaces of points and lines.

Our main contention is that all the trigonometry of the space is completely contained in these equations, which have as a remarkable property their explicit duality under the interchange $a, b, c \leftrightarrow A, B, C$ and $P \leftrightarrow J$; this duality is a consequence of the fact that $\mathcal{D}(2.8)$ is an automorphism of the family of CK algebras which interchanges $P_{1} \leftrightarrow-J_{12}$, and therefore $a, b, c \leftrightarrow-A,-B,-C$.

The first equation in (3.1) gives the translation generator $P_{b}$ as a conjugate of $P_{a}$ by means of a rotation around the vertex $C$; the same equation read inversely gives $P_{a}$ as a conjugate of $P_{b}$ by means of the inverse rotation around $C$. These expressions will be utilised several times in the ongoing derivations, and we will refer to them as $P_{b}\left(P_{a}\right)$ or $P_{a}\left(P_{b}\right)$; likewise the two remaining equations for the translations will be referred to as $P_{c}\left(P_{b}\right)$ and $P_{a}\left(P_{b}\right)$. Similar shorthands $J_{B}\left(J_{A}\right)$, etc. will be used to make reference to the three equations relating the rotation generators.

By cyclic substitution in the three equations $P_{a}\left(P_{c}\right), P_{c}\left(P_{b}\right)$ and $P_{b}\left(P_{a}\right)$ we find the identity

$$
e^{B J_{B}} e^{-A J_{A}} e^{C J_{C}} P_{a} e^{-C J_{C}} e^{A J_{A}} e^{-B J_{B}}=P_{a}
$$

as well as two similar equations for $P_{b}$ and $P_{c}$, obtained by starting the substitution in either $P_{b}$ or $P_{c}$. Likewise, a dual completely parallel process allows us to derive analogous identities for $J_{A}$ and $J_{B}$, which are similar to the identity involving $J_{C}$ :

$$
e^{-a P_{a}} e^{c P_{c}} e^{b P_{b}} J_{C} e^{-b P_{b}} e^{-c P_{c}} e^{a P_{a}}=J_{C} .
$$


Equations (3.2) and (3.3) can be written alternatively as:

$$
\begin{aligned}
& e^{B J_{B}} e^{-A J_{A}} e^{C J_{C}} \text { must commute with } P_{a}, \\
& e^{-a P_{a}} e^{c P_{c}} e^{b P_{b}} \text { must commute with } J_{C} .
\end{aligned}
$$

\subsection{Loop excesses and loop equations}

The content of (3.4) is transparent and could indeed have been taken as the starting point, as the following alternative reasoning shows: the product $e^{-a P_{a}} e^{c P_{c}} e^{b P_{b}}$ of the three translations along the three sides of the triangle $C \stackrel{b}{\rightarrow} A \stackrel{c}{\rightarrow} B \stackrel{-a}{\rightarrow} C$ moves the base point $C$ along the triangle and returns it back to its original position, so it must necessarily be a rotation around the vertex $C$ by some angle $-\Delta_{C}$ :

$$
e^{-a P_{a}} e^{c P_{c}} e^{b P_{b}}=e^{-\Delta_{C} J_{C}} .
$$

Therefore the product $e^{-a P_{a}} e^{c P_{c}} e^{b P_{b}}$ must commute with $J_{C}$. Likewise, the product $e^{B J_{B}} e^{-A J_{A}} e^{C J_{C}}$ of the three rotations around the three vertices, $a \stackrel{C}{\rightarrow} b \stackrel{-A}{\rightarrow} c \stackrel{B}{\rightarrow} a$ must be a translation along the side $a$ by an amount $-\delta_{a}$ :

$$
e^{B J_{B}} e^{-A J_{A}} e^{C J_{C}}=e^{-\delta_{a} P_{a}} .
$$

The two quantities $\delta_{a}$ and $\Delta_{C}$ (as well as their analogous $\delta_{b}, \delta_{c}$ and $\Delta_{A}, \Delta_{B}$ which appear in equations similar to (3.5) and (3.6)) are so far unknown, but they are completely determined by the triangle loop. To find them we start with the equation which gives $P_{c}\left(P_{b}\right)$ in the set $(3.1)$, replace $P_{c}$ by $e^{-c P_{c}} P_{c} e^{c P_{c}}$ and then substitute $P_{c}\left(P_{a}\right)$ from the compatibility equations to obtain:

$$
e^{-A J_{A}} P_{b} e^{A J_{A}}=e^{-c P_{c}} e^{-B J_{B}} P_{a} e^{B J_{B}} e^{c P_{c}} .
$$

We introduce $J_{B}\left(J_{C}\right)$ and trivially simplify to obtain:

$$
e^{-A J_{A}} P_{b} e^{A J_{A}}=e^{-c P_{c}} e^{a P_{a}} e^{-B J_{C}} P_{a} e^{B J_{C}} e^{-a P_{a}} e^{c P_{c}}
$$

which is equivalent to

$$
e^{B J_{C}} e^{-a P_{a}} e^{c P_{c}} e^{-A J_{A}} P_{b} e^{A J_{A}} e^{-c P_{c}} e^{a P_{a}} e^{-B J_{C}}=P_{a} .
$$

Now we use $J_{A}\left(J_{C}\right)$, simplify, and finally substitute $P_{b}\left(P_{a}\right)$. This gives:

$$
e^{B J_{C}} e^{-a P_{a}} e^{c P_{c}} e^{b P_{b}} e^{-A J_{C}} e^{C J_{C}} P_{a} e^{-C J_{C}} e^{A J_{C}} e^{-b P_{b}} e^{-c P_{c}} e^{a P_{a}} e^{-B J_{C}}=P_{a} .
$$

Note that the three translations along the triangle appear in a single piece $e^{-a P_{a}} e^{c P_{c}} e^{b P_{b}}$, while the three rotations are all around the base point $C$. Now we can go a bit further: (3.4) implies that $e^{-a P_{a}} e^{c P_{c}} e^{b P_{b}}$ must commute with $J_{C}$, so it will commute with any rotation around $C$. Then we can commute the whole piece $e^{-a P_{a}} e^{c P_{c}} e^{b P_{b}}$ in (3.10) with the rotations around $C$ and collect these altogether. Thus:

$$
e^{-a P_{a}} e^{c P_{c}} e^{b P_{b}} e^{(-A+B+C) J_{C}} P_{a} e^{-(-A+B+C) J_{C}} e^{-b P_{b}} e^{-c P_{c}} e^{a P_{a}}=P_{a}
$$

or

$$
e^{-a P_{a}} e^{c P_{c}} e^{b P_{b}} e^{(-A+B+C) J_{C}} \text { must commute with } P_{a} .
$$


Since we had already derived that the expression $e^{-a P_{a}} e^{c P_{c}} e^{b P_{b}} e^{X J_{C}}$ must commute with $J_{C}$ for any angle $X$ (see (3.4)), and this expression also commutes with $P_{a}$ for the special value $X=-A+B+C$, we immediately conclude that,

$$
e^{-a P_{a}} e^{c P_{c}} e^{b P_{b}} e^{(-A+B+C) J_{C}}=1
$$

since the identity is the only element of $S O_{\kappa_{1}, \kappa_{2}}(3)$ that commutes with two such generators as $P_{a}$ and $J_{C}$. This equation can be also written as:

$$
e^{-a P_{a}} e^{c P_{c}} e^{b P_{b}}=e^{-(-A+B+C) J_{C}}
$$

and so it gives the unknown angle $\Delta_{C}=-A+B+C$ appearing in (3.5). A very similar procedure (or direct use of (3.1) in (3.14)) allows us to derive two analogous equations:

$$
\begin{aligned}
& e^{b P_{b}} e^{-a P_{a}} e^{c P_{c}}=e^{-(-A+B+C) J_{A}} \\
& e^{c P_{c}} e^{b P_{b}} e^{-a P_{a}}=e^{-(-A+B+C) J_{B}}
\end{aligned}
$$

hence we obtain

$$
\Delta_{A}=\Delta_{B}=\Delta_{C}=-A+B+C \equiv \Delta .
$$

The quantity $\Delta$ will be called the angular excess of the triangle loop, and fits very naturally into the view of the point loop as a single curve which starts on the geodesic $a$, and successively rotates by angles $C,-A$ and $B$ around the three vertices of the triangle, so that $-A+B+C$ should be looked as the (oriented) total angle turned by the line loop. Equations (3.14) or (3.15), to be called the point loop equations, simply state that the product of the three translations along the oriented sides of the triangle loop equals a rotation around the base point of the loop, with an angle equal to minus the angular excess of the triangle loop.

The explicit duality of the starting equations (3.1) under the interchange $a, b, c \leftrightarrow$ $A, B, C$ and $P \leftrightarrow J$ immediately implies that the dual process leads, mutatis mutandis, to the dual partners of equations (3.14) and (3.15):

$$
\begin{aligned}
& e^{-A J_{A}} e^{C J_{C}} e^{B J_{B}}=e^{-(-a+b+c) P_{c}} \\
& e^{B J_{B}} e^{-A J_{A}} e^{C J_{C}}=e^{-(-a+b+c) P_{a}} \\
& e^{C J_{C}} e^{B J_{B}} e^{-A J_{A}}=e^{-(-a+b+c) P_{b}}
\end{aligned}
$$

implying that

$$
\delta_{a}=\delta_{b}=\delta_{c}=-a+b+c \equiv \delta
$$

which will be called the lateral excess of the triangle. This appears as the (oriented) total length of the point loop, where $b$ and $c$ are traversed in the same sense of the orientations chosen for $P_{b}, P_{c}$, but $a$ is traversed backwards relative to $P_{a}$. Therefore (3.17), to be called line loop equations, gives the product of the three oriented rotations around the three vertices of a triangle as a translation along the base line of the loop, by an amount equal to minus the lateral excess of the triangle loop.

Consequently, the canonical parameters of the 'holonomy' rotation, or of the dual 'holonomy' translation are independent of the base point or line, and are therefore associated to the triangle loop in an intrinsic way. The excesses $\Delta$ and $\delta$ are directly related to other natural quantities, the area and coarea of the triangular loop. 


\subsection{The basic trigonometric identity}

Potentially, each of the equations (3.14), (3.15) and (3.17) contains all the trigonometry of any CK space, that is, all the relationships between the three sides and the three angles of the triangle. However, sides and angles appear in these equations not only explicitly as canonical parameters, but also implicitly hidden inside the translation and rotation generators. This prompts the search for another relation, equivalent to the previous ones but more suitable to display the trigonometric equations; this new equation is indeed the bridge between the former equations and the trigonometry of the space. The main idea is to express all the generators as suitable conjugates of one translation generator and one rotation generator, which we will take as primitive independent generators; the choice is only restricted by the flag condition that the center of the rotation should lie on the axis of the translation.

A natural choice is to take $P_{a}$ and $J_{C}$ as 'basic' independent generators. Next by using the compatibility conditions (3.1) we define the remaining triangle generators $P_{b}, J_{A}, P_{c}, J_{B}$ in term of the previous ones and the values of sides and angles as:

$$
\begin{aligned}
& P_{b}:=e^{C J_{C}} P_{a} e^{-C J_{C}} \\
& J_{A}:=e^{b P_{b}} J_{C} e^{-b P_{b}} \\
& P_{c}:=e^{-A J_{A}} P_{b} e^{A J_{A}} \\
& J_{B}:=e^{c P_{c}} J_{A} e^{-c P_{c}}
\end{aligned}
$$

which after full expansion and simplification gives:

$$
\begin{aligned}
& P_{b}:=e^{C J_{C}} P_{a} e^{-C J_{C}} \\
& J_{A}:=e^{C J_{C}} e^{b P_{a}} J_{C} e^{-b P_{a}} e^{-C J_{C}} \\
& P_{c}:=e^{C J_{C}} e^{b P_{a}} e^{-A J_{C}} P_{a} e^{A J_{C}} e^{-b P_{a}} e^{-C J_{C}} \\
& J_{B}:=e^{C J_{C}} e^{b P_{a}} e^{-A J_{C}} e^{c P_{a}} J_{C} e^{-c P_{a}} e^{A J_{C}} e^{-b P_{a}} e^{-C J_{C}} \text {. }
\end{aligned}
$$

(Note the highly ordered pattern in these expressions). By direct substitution in the equation (3.14) we obtain

$$
e^{-a P_{a}} \cdot e^{C J_{C}} e^{b P_{a}} e^{-A J_{C}} e^{c P_{a}} e^{A J_{C}} e^{-b P_{a}} e^{-C J_{C}} \cdot e^{C J_{C}} e^{b P_{a}} e^{-C J_{C}}=e^{-(-A+B+C) J_{C}}
$$

which after obvious cancellations gives:

$$
e^{-a P_{a}} e^{C J_{C}} e^{b P_{a}} e^{-A J_{C}} e^{c P_{a}} e^{B J_{C}}=1 .
$$

On the other hand, substitution of (3.20) in the second equation in (3.17) and simplification gives

$$
e^{C J_{C}} e^{b P_{a}} e^{-A J_{C}} e^{c P_{a}} e^{B J_{C}} e^{-a P_{a}}=1
$$

which coincides with the previous equation, because the terms can be cyclically permuted. The same process starting from any of the two equations (3.15) which are associated to the other two base points in the triangle, or any of the equations in (3.17) but associated to the other two base lines in the triangle, leads again to the same equation. This justifies to call (3.22) the basic trigonometric equation.

It is possible to give an heuristic derivation of (3.22). Consider a kind of complete group motion associated to the triangle:

$$
e^{-a P_{a}} e^{B J_{B}} e^{c P_{c}} e^{-A J_{A}} e^{b P_{b}} e^{C J_{C}} .
$$


It is clear that this group element leaves fixed both the base line $a$ and the base point $C$. Therefore it should be equal to the identity:

$$
e^{-a P_{a}} e^{B J_{B}} e^{c P_{c}} e^{-A J_{A}} e^{b P_{b}} e^{C J_{C}}=1 .
$$

Now substitution of (3.20) in (3.25) and obvious cancellations gives directly the basic equation (3.22). However, this reasoning line does not display the relevance of loop excesses. The equivalence between the equation (3.25), which involves the three translation generators $P_{a}, P_{b}, P_{c}$ with parameters $-a, b, c$ and the three rotation generators $J_{A}, J_{B}, J_{C}$ with angles $-A, B, C$, and the equation (3.22), where only the fiducial generators $P_{a}$ and $J_{C}$ appear with the same parameters, is an apparently surprising but obvious consequence of the compatibility conditions.

The results obtained so far can be summed up in the following:

Theorem 1. Sides $a, b, c$ and angles $A, B, C$ of any triangle loop are linked by the single group identity called the basic trigonometric identity

$$
e^{-a P} e^{C J} e^{b P} e^{-A J} e^{c P} e^{B J}=1
$$

where $P, J$ are the generators of translations along any fixed fiducial line $l$, and of rotations around any fixed fiducial point $O$ on the line $l$.

Proof: A group motion can be used to move any triangle to a canonical position where the vertex $C$ lies on the fiducial point $O$, and the side $a$ lies on the fiducial line $l$. Then the theorem statement is simply the equation (3.22).

Theorem 2. Let $P_{a}, P_{b}, P_{c}$ be the generators of translations along the three sides of a triangle (whose lengths are $a, b, c$ ), and $J_{A}, J_{B}, J_{C}$ the generators of rotations around the three vertices (with angles $A, B, C$ ). Then we have two sets of identities, called the point loop and the line loop equations for the triangle:

$$
\begin{array}{lll}
e^{-a P_{a}} e^{c P_{c}} e^{b P_{b}}=e^{-(-A+B+C) J_{C}} & e^{B J_{B}} e^{-A J_{A}} e^{C J_{C}}=e^{-(-a+b+c) P_{a}} \\
e^{b P_{b}} e^{-a P_{a}} e^{c P_{c}}=e^{-(-A+B+C) J_{A}} & e^{C J_{C}} e^{B J_{B}} e^{-A J_{A}}=e^{-(-a+b+c) P_{b}} \\
e^{c P_{c}} e^{b P_{b}} e^{-a P_{a}}=e^{-(-A+B+C) J_{B}} & e^{-A J_{A}} e^{C J_{C}} e^{B J_{B}}=e^{-(-a+b+c) P_{c}} .
\end{array}
$$

Furthermore each of these identities is equivalent to the identity in Theorem 1.

As each point loop and each line loop equation is equivalent to the basic identity, we conclude that the three point loop equations, the three line loop equations and the basic equation are all equivalent.

Several points are worth highlighting. First, each term in the basic identity is either a translation along a fixed line $l$ or a rotation around a fixed point $O$, so any relation with the original triangle translation or rotation generators $P_{a}, P_{b}, P_{c}$ or $J_{A}, J_{B}, J_{C}$ is somewhat concealed. However, the canonical parameters of these fiducial translations or rotations are exactly the sides and the angles of the triangle. In the point loop or line loop equations, however, the transformations involved are the translations along the three sides or the rotations around the three vertices.

Second, the structure of the equations can be easily remembered. The point loop equations follow from a point travelling along the triangle; it starts at the base point $C$ 
and follows the sides in the order $b, c,-a$, with $a$ negative as it is traversed backwards (see figure 2a). The line loop equations follow from a line looping around the triangle; it starts in a base line (say $a$ ) and successively rotates by an angle $C$, then by an angle $-A$ and finally by an angle $B$ around the corresponding vertices thus ending up back on the starting position $a$ (see figure $2 \mathrm{~b}$ ). The basic equation follows from the pattern $-a, C, b,-A, c, B$, which keeps track of both sides and vertices found when looping around the triangle; again $a, A$ appear with a minus sign, because both are traversed in negative sense.

Third, the (three) point loop equations and the (three) line loop equations are mutually dual sets. The single basic equation is clearly self-dual.

Fourth, and worth some emphasizing, these equations hold in the same explicit form for all 2D real CK geometries, as no explicit $\kappa_{1}, \kappa_{2}$ ever appear in them. In the well known euclidean case, the angle sum theorem for a triangle - which reads $A=B+C$ in terms of our external angle $A$ - is usually taken as an elementary property of the geometry, known beforehand trigonometry, and in this particular space the point loop equation reduces to $e^{-a P_{a}} e^{c P_{c}} e^{b P_{b}}=1$. This simply says that no holonomy rotation is associated to the triangle at all, and conveys the flatness of the euclidean plane. In our approach the holonomy angle is always $-A+B+C$ (no matter of the values of $\kappa_{1}, \kappa_{2}$ ), but whether or not this angle vanishes actually depends on the values of $\kappa_{1}, \kappa_{2}$; as we shall see the equations of trigonometry themselves imply the vanishing of $-A+B+C$ for $\kappa_{1}=0$. In other words, the euclidean relation $A=B+C$ as well as their non-linear non-euclidean analogous will arise as part of the trigonometric relations.

\section{The basic equations of trigonometry in the nine Cayley- Klein spaces}

The most convenient way to obtain the trigonometric equations is to start with the basic trigonometry identity (3.26), in which from now on the two generators $P$ and $J$ will be taken to be exactly $P_{1}$ and $J_{12}$. For convenience, we write (3.26) as

$$
e^{-a P} e^{C J} e^{b P}=e^{-B J} e^{-c P} e^{A J} .
$$

By considering this identity in the fundamental 3D vector representation of the motion group (2.13) we obtain an equality between $3 \times 3$ matrices, giving rise to nine identities:

$$
\begin{array}{ll}
1 \mathrm{c} & C_{\kappa_{1}}(c)=C_{\kappa_{1}}(a) C_{\kappa_{1}}(b)+\kappa_{1} S_{\kappa_{1}}(a) S_{\kappa_{1}}(b) C_{\kappa_{2}}(C) \\
1 \mathrm{C} & C_{\kappa_{2}}(C)=C_{\kappa_{2}}(A) C_{\kappa_{2}}(B)+\kappa_{2} S_{\kappa_{2}}(A) S_{\kappa_{2}}(B) C_{\kappa_{1}}(c) \\
2 \mathrm{cA} \equiv 2 \mathrm{aC} & S_{\kappa_{1}}(c) S_{\kappa_{2}}(A)=S_{\kappa_{1}}(a) S_{\kappa_{2}}(C) \\
2 \mathrm{cB} \equiv 2 \mathrm{bC} & S_{\kappa_{1}}(c) S_{\kappa_{2}}(B)=S_{\kappa_{1}}(b) S_{\kappa_{2}}(C) \\
3 \mathrm{cA} & S_{\kappa_{1}}(c) C_{\kappa_{2}}(A)=-C_{\kappa_{1}}(a) S_{\kappa_{1}}(b)+S_{\kappa_{1}}(a) C_{\kappa_{1}}(b) C_{\kappa_{2}}(C) \\
3 \mathrm{cB} & S_{\kappa_{1}}(c) C_{\kappa_{2}}(B)=C_{\kappa_{1}}(b) S_{\kappa_{1}}(a)-S_{\kappa_{1}}(b) C_{\kappa_{1}}(a) C_{\kappa_{2}}(C) \\
3 \mathrm{Ca} & S_{\kappa_{2}}(C) C_{\kappa_{1}}(a)=-C_{\kappa_{2}}(A) S_{\kappa_{2}}(B)+S_{\kappa_{2}}(A) C_{\kappa_{2}}(B) C_{\kappa_{1}}(c) \\
3 \mathrm{Cb} & S_{\kappa_{2}}(C) C_{\kappa_{1}}(b)=C_{\kappa_{2}}(B) S_{\kappa_{2}}(A)-S_{\kappa_{2}}(B) C_{\kappa_{2}}(A) C_{\kappa_{1}}(c) \\
4 \mathrm{AB} \equiv 4 \mathrm{ab} & \kappa_{2} S_{\kappa_{2}}(A) S_{\kappa_{2}}(B)+C_{\kappa_{2}}(A) C_{\kappa_{2}}(B) C_{\kappa_{1}}(c) \\
& =\kappa_{1} S_{\kappa_{1}}(a) S_{\kappa_{1}}(b)+C_{\kappa_{1}}(a) C_{\kappa_{1}}(b) C_{\kappa_{2}}(C)
\end{array}
$$

which are the equations for the trigonometry of the space $S_{\left[\kappa_{1}\right], \kappa_{2}}^{2}=S O_{\kappa_{1}, \kappa_{2}}(3) / S O_{\kappa_{2}}(2)$. The tag assigned to each equation is self-descriptive: all equations are either self-dual (for 
instance $2 \mathrm{cA} \equiv 2 \mathrm{aC}$ ) or appear in mutually dual pairs (as $(1 \mathrm{c}, 1 \mathrm{C})$ or $(3 \mathrm{cA}, 3 \mathrm{Ca})$ ); this could have been expected due to the self-duality of the starting equation.

A first elementary but remarkable property of these equations is the complete matching of sides and angles with the two labels $\kappa_{1}$ and $\kappa_{2}$; all sides (resp. angles) appear in the equations through the trigonometric functions which have $\kappa_{1}$ (resp. $\kappa_{2}$ ) as a label. Then the association between labels and generators extends to the lengths and angles themselves, and we will say that first-kind lengths have $\kappa_{1}$ (resp. angles $\kappa_{2}$ ) as a label.

The two equations $2 \mathrm{cA}, 2 \mathrm{cB}$ taken together are the sine theorem, whose general formulation for the nine CK spaces displays explicit self-duality (cf. (4.9)). Next, the equation $1 \mathrm{c}$ is the cosine theorem for the side $c$, and $1 \mathrm{C}$ is the dual cosine theorem for the angle $C$; a pair of relationships whose mutual duality can be clearly seen in this general approach. The two equations $3 \mathrm{cA}, 3 \mathrm{cB}$ can be considered as two 'addition' formulas for the side $c$ in terms of the projections of the others, and the two formulas $3 \mathrm{Ca}, 3 \mathrm{Cb}$ are their duals. The single self-dual equation $4 \mathrm{AB}$ relates all six trigonometric quantities.

To describe clearly the structure and dependence between these equations, it is better to consider the group of equations (4.2) altogether with two similar groups, each equivalent as a set of equations to the previous one. These can be obtained by starting from the basic identity written in two alternative forms:

$$
e^{b P} e^{-A J} e^{c P} e^{B J} e^{-a P} e^{C J}=1 \quad e^{c P} e^{B J} e^{-a P} e^{C J} e^{b P} e^{-A J}=1
$$

Spliting them again as

$$
e^{b P} e^{-A J} e^{c P}=e^{-C J} e^{a P} e^{-B J} \quad e^{c P} e^{B J} e^{-a P}=e^{A J} e^{-b P} e^{-C J}
$$

and writing them in the fundamental representation (2.13), we obtain other two sets of equations very similar to (4.2). We only write the two first equations for each set:

$$
\begin{array}{lc}
1 \mathrm{a} & C_{\kappa_{1}}(a)=C_{\kappa_{1}}(b) C_{\kappa_{1}}(c)-\kappa_{1} S_{\kappa_{1}}(b) S_{\kappa_{1}}(c) C_{\kappa_{2}}(A) \\
1 \mathrm{~A} & C_{\kappa_{2}}(A)=C_{\kappa_{2}}(B) C_{\kappa_{2}}(C)-\kappa_{2} S_{\kappa_{2}}(B) S_{\kappa_{2}}(C) C_{\kappa_{1}}(a) \\
\vdots & \vdots \\
& \\
1 \mathrm{~b} & C_{\kappa_{1}}(b)=C_{\kappa_{1}}(a) C_{\kappa_{1}}(c)+\kappa_{1} S_{\kappa_{1}}(a) S_{\kappa_{1}}(c) C_{\kappa_{2}}(B) \\
\text { 1B } & C_{\kappa_{2}}(B)=C_{\kappa_{2}}(A) C_{\kappa_{2}}(C)+\kappa_{2} S_{\kappa_{2}}(A) S_{\kappa_{2}}(C) C_{\kappa_{1}}(b)
\end{array}
$$

All the equations (4.2), (4.5) and (4.6) taken altogether can be grouped into:

- Three cosine theorems for sides:

$$
\begin{array}{ll}
\text { 1a } & C_{\kappa_{1}}(a)=C_{\kappa_{1}}(b) C_{\kappa_{1}}(c)-\kappa_{1} S_{\kappa_{1}}(b) S_{\kappa_{1}}(c) C_{\kappa_{2}}(A) \\
\text { 1b } & C_{\kappa_{1}}(b)=C_{\kappa_{1}}(a) C_{\kappa_{1}}(c)+\kappa_{1} S_{\kappa_{1}}(a) S_{\kappa_{1}}(c) C_{\kappa_{2}}(B) \\
\text { 1c } & C_{\kappa_{1}}(c)=C_{\kappa_{1}}(a) C_{\kappa_{1}}(b)+\kappa_{1} S_{\kappa_{1}}(a) S_{\kappa_{1}}(b) C_{\kappa_{2}}(C)
\end{array}
$$

and three dual cosine theorems for angles:

$$
\begin{array}{ll}
1 \mathrm{~A} & C_{\kappa_{2}}(A)=C_{\kappa_{2}}(B) C_{\kappa_{2}}(C)-\kappa_{2} S_{\kappa_{2}}(B) S_{\kappa_{2}}(C) C_{\kappa_{1}}(a) \\
\text { 1B } & C_{\kappa_{2}}(B)=C_{\kappa_{2}}(A) C_{\kappa_{2}}(C)+\kappa_{2} S_{\kappa_{2}}(A) S_{\kappa_{2}}(C) C_{\kappa_{1}}(b) \\
1 \mathrm{C} & C_{\kappa_{2}}(C)=C_{\kappa_{2}}(A) C_{\kappa_{2}}(B)+\kappa_{2} S_{\kappa_{2}}(A) S_{\kappa_{2}}(B) C_{\kappa_{1}}(c) .
\end{array}
$$


- One self-dual sine theorem:

$$
2 \quad \frac{S_{\kappa_{1}}(a)}{S_{\kappa_{2}}(A)}=\frac{S_{\kappa_{1}}(b)}{S_{\kappa_{2}}(B)}=\frac{S_{\kappa_{1}}(c)}{S_{\kappa_{2}}(C)} .
$$

- Six 'side addition' theorems which correspond to the tags $3 \mathrm{aB}, 3 \mathrm{aC}, 3 \mathrm{bA}, 3 \mathrm{bC}, 3 \mathrm{cA}, 3 \mathrm{cB}$ and six dual 'angle addition' theorems $3 \mathrm{Ab}, 3 \mathrm{Ac}, 3 \mathrm{Ba}, 3 \mathrm{Bc}, 3 \mathrm{Ca}, 3 \mathrm{Cb}$.

- And three self-dual theorems $4 \mathrm{AB} \equiv 4 \mathrm{ab}, 4 \mathrm{AC} \equiv 4 \mathrm{ac}, 4 \mathrm{BC} \equiv 4 \mathrm{bc}$.

We notice the signs in $1 \mathrm{a}, 1 \mathrm{~A}$, differing from those in $1 \mathrm{~b}, 1 \mathrm{~B}$ and $1 \mathrm{c}, 1 \mathrm{C}$. The same sign difference occurs in all other equations, and can be traced back to the appearance of $-a$ and $-A$ in the basic equation, as compared with $b, c$ and $B, C$.

These equations are a complete set of trigonometric equations for any values of the constants $\kappa_{1}, \kappa_{2}$, but most of them reduce to simpler, or even trivial ones in the degenerate cases $\kappa_{1}=0$ or $\kappa_{2}=0$.

\subsection{Alternative forms for the cosine theorems}

The cosine theorems (4.7) (resp. (4.8)) give rise to trivial identities $1=1$ when $\kappa_{1}=0$ (resp. $\kappa_{2}=0$ ). This can be circumvented by writing these equations in an alternative form. Take the cosine equation for the side $c(1 \mathrm{c}$ of $(4.7))$ in the generic case with $\kappa_{1} \neq 0$, and write all cosines in terms of versed sines by introducing (A.2):

$$
1-\kappa_{1} V_{\kappa_{1}}(c)=\left(1-\kappa_{1} V_{\kappa_{1}}(a)\right)\left(1-\kappa_{1} V_{\kappa_{1}}(b)\right)+\kappa_{1} S_{\kappa_{1}}(a) S_{\kappa_{1}}(b)\left(1-\kappa_{2} V_{\kappa_{2}}(C)\right) .
$$

By expanding and cancelling a common factor $\kappa_{1}$ we find that

$$
V_{\kappa_{1}}(c)=V_{\kappa_{1}}(a)+V_{\kappa_{1}}(b)-\kappa_{1} V_{\kappa_{1}}(a) V_{\kappa_{1}}(b)-S_{\kappa_{1}}(a) S_{\kappa_{1}}(b)+\kappa_{2} S_{\kappa_{1}}(a) S_{\kappa_{1}}(b) V_{\kappa_{2}}(C),
$$

which is rather simplified by means of (A.10). The remaining cosine theorems 1a, 1b and the dual cosine equations (4.8) allow a similar reformulation. Thus we obtain the following alternative expressions:

$$
\begin{array}{rlrl}
1 ' \mathrm{a} & V_{\kappa_{1}}(a)-V_{\kappa_{1}}(b+c) & =-\kappa_{2} S_{\kappa_{1}}(b) S_{\kappa_{1}}(c) V_{\kappa_{2}}(A) \\
1 ' \mathrm{~b} & V_{\kappa_{1}}(b)-V_{\kappa_{1}}(a-c) & =\kappa_{2} S_{\kappa_{1}}(a) S_{\kappa_{1}}(c) V_{\kappa_{2}}(B) \\
1 ' \mathrm{c} & V_{\kappa_{1}}(c)-V_{\kappa_{1}}(a-b) & =\kappa_{2} S_{\kappa_{1}}(a) S_{\kappa_{1}}(b) V_{\kappa_{2}}(C) \\
& & & \\
1 ' \mathrm{~A} & V_{\kappa_{2}}(A)-V_{\kappa_{2}}(B+C) & =-\kappa_{1} S_{\kappa_{2}}(B) S_{\kappa_{2}}(C) V_{\kappa_{1}}(a) \\
1 ' \mathrm{~B} & V_{\kappa_{2}}(B)-V_{\kappa_{2}}(A-C) & =\kappa_{1} S_{\kappa_{2}}(A) S_{\kappa_{2}}(C) V_{\kappa_{1}}(b) \\
\text { 1'C } & V_{\kappa_{2}}(C)-V_{\kappa_{2}}(A-B) & =\kappa_{1} S_{\kappa_{2}}(A) S_{\kappa_{2}}(B) V_{\kappa_{1}}(c) .
\end{array}
$$

These relations are clearly equivalent to $(4.7)($ resp. $(4.8))$ when $\kappa_{1} \neq 0\left(\right.$ resp. $\kappa_{2} \neq 0$ ), but do not reduce to trivial identities when $\kappa_{1}=0$ or $\kappa_{2}=0$. In this sense they can be considered as a 'good' form of cosine and dual cosine equations.

Equations (4.12) and (4.13) still allow another alternative very useful form. Consider the half sums of the three sides and of the three angles (cf. (A.17)),

$$
p=\frac{a+b+c}{2} \quad P=\frac{A+B+C}{2} .
$$


By introducing the identities (A.28) and (A.29) applied to the three sides $a, b, c$ and angles $A, B, C$ into the cosine theorems (4.12) and (4.13), we obtain

$$
\begin{array}{rl}
1 " \mathrm{a} & 2 S_{\kappa_{1}}(p-a) S_{\kappa_{1}}(p)=\kappa_{2} S_{\kappa_{1}}(b) S_{\kappa_{1}}(c) V_{\kappa_{2}}(A) \\
1 \text { "b } & 2 S_{\kappa_{1}}(p-a) S_{\kappa_{1}}(p-c)=\kappa_{2} S_{\kappa_{1}}(a) S_{\kappa_{1}}(c) V_{\kappa_{2}}(B) \\
1 " \mathrm{c} & 2 S_{\kappa_{1}}(p-a) S_{\kappa_{1}}(p-b)=\kappa_{2} S_{\kappa_{1}}(a) S_{\kappa_{1}}(b) V_{\kappa_{2}}(C) \\
& \\
1 \text { " A } & 2 S_{\kappa_{2}}(P-A) S_{\kappa_{2}}(P)=\kappa_{1} S_{\kappa_{2}}(B) S_{\kappa_{2}}(C) V_{\kappa_{1}}(a) \\
1 \text { 1" } & 2 S_{\kappa_{2}}(P-A) S_{\kappa_{2}}(P-C)=\kappa_{1} S_{\kappa_{2}}(A) S_{\kappa_{2}}(C) V_{\kappa_{1}}(b) \\
1 \text { 1" } & 2 S_{\kappa_{2}}(P-A) S_{\kappa_{2}}(P-B)=\kappa_{1} S_{\kappa_{2}}(A) S_{\kappa_{2}}(B) V_{\kappa_{1}}(c) .
\end{array}
$$

\subsection{Dependence and sets of basic equations}

The equations we have obtained so far contain the whole trigonometry of the CK space $S_{\left[\kappa_{1}\right], \kappa_{2}}^{2} \equiv S O_{\kappa_{1}, \kappa_{2}}(3) / S O_{\kappa_{2}}(2)$ and hold for any of the nine CK geometries by simply particularising the real coefficients $\kappa_{i}$ to their values for each geometry. Not all these equations can be independent: in any $\mathrm{CK}$ space, a triangle is completely determined by three independent quantities (e.g. $a, b, C$, because the side-angle-side (SAS) congruence condition clearly holds in all the nine cases) so we should expect three independent relations between the six quantities $a, b, c ; A, B, C$.

Let us first discuss the case with $\kappa_{1}=0$ but $\kappa_{2} \neq 0$. In these degenerate cases we obtain the well known trigonometry of the euclidean plane $\left(\kappa_{2}>0\right)$, and the less well known lorentzian trigonometry of the $(1+1) \mathrm{D}$ minkowskian spacetime $\left(\kappa_{2}<0\right)$ [1]. The formulas (4.7) reduce to trivial identities $1=1$, but the alternative expressions (4.12) or (4.15) lead to the three ordinary flat euclidean or lorentzian cosine theorems, the latter exactly as given in [1]. For instance, 1'c gives rise to

$$
\frac{1}{2} c^{2}-\frac{1}{2}(a-b)^{2}=\kappa_{2} a b V_{\kappa_{2}}(C) \equiv c^{2}=a^{2}+b^{2}-2 a b C_{\kappa_{2}}(C) .
$$

All the remaining equations of trigonometry do not reduce to identities and are directly meaningful, yet simpler. By taking into account the sine and cosine addition identities (A.8) and (A.9), we find that for $\kappa_{1}=0$ the content of all the dual cosine theorems (4.8) and all equations $3 \mathrm{Ab}, 3 \mathrm{Ac}, 3 \mathrm{Ba}, 3 \mathrm{Bc}, 3 \mathrm{Ca}, 3 \mathrm{Cb}, 4 \mathrm{AB}, 4 \mathrm{AC}, 4 \mathrm{BC}$ is the same and reduces to a triangular angle addition in the form:

$$
A=B+C \equiv \Delta=0 .
$$

The equality $\Delta=0$ implies that for $\kappa_{1}=0$ the holonomy (3.14) is equal to the identity, as it should in any flat space. In these flat spaces where $\kappa_{1}=0$ the angles are related by a 'universal' linear equation not depending on the sides. This universality is why the equality $A=B+C$ is usually taken as a property of euclidean geometry, and not as a trigonometric equation. The sine theorem (4.9) reads now

$$
\frac{a}{S_{\kappa_{2}}(A)}=\frac{b}{S_{\kappa_{2}}(B)}=\frac{c}{S_{\kappa_{2}}(C)}
$$

and the remaining equations $3 \mathrm{aB}, 3 \mathrm{aC}, 3 \mathrm{bA}, 3 \mathrm{bC}, 3 \mathrm{cA}, 3 \mathrm{cB}$ give each side as the sum of the projections of the other two. In particular, relations $3 \mathrm{cA}$ and $3 \mathrm{cB}$ in (4.2) reduce to:

$$
b=a C_{\kappa_{2}}(C)-c C_{\kappa_{2}}(A) \quad a=b C_{\kappa_{2}}(C)+c C_{\kappa_{2}}(B) .
$$


Notice that the three cosine theorems 1'a, 1'b, 1'c (as (4.17)) are still independent. From these we can derive all the remaining non-trivial equations, including the dual cosine theorem (4.18), the sine theorem (4.19) (see below) and the relations on sum of projections as (4.20). Henceforth, when $\kappa_{1}=0$ but $\kappa_{2} \neq 0$, the canonical choice for three independent equations is the three alternative cosine theorems (4.12) for the three sides.

A fully parallel dual discussion can be repeated for the case $\kappa_{2}=0$ but $\kappa_{1} \neq 0$. Then the equations (4.8) give rise to trivial identities, while (4.13) or (4.16) provide the dual cosine theorems for the angles, which are the three independent equations. The relations (4.7), $3 \mathrm{aB}, 3 \mathrm{aC}, 3 \mathrm{bA}$, etc. reduce to the same single equation:

$$
a=b+c \equiv \delta=0
$$

Finally, in the more contracted case with $\kappa_{1}=\kappa_{2}=0$, that is, the $(1+1) \mathrm{D}$ galilean geometry, the equations are simply

$$
a=b+c \quad A=B+C \quad \frac{a}{A}=\frac{b}{B}=\frac{c}{C}
$$

which are fully linear. The three independent equations are a single addition theorem for sides alone, another for angles alone, and a 'sine' theorem stating the proportionality of the three sides and angles.

All the results about the dependence of equations can be summed up in the following:

Theorem 3. The full set of equations of trigonometry always contains, (i.e. for any value of $\kappa_{1}, \kappa_{2}$ ) exactly three independent equations. Any other equation in the set is a consequence of them. According to the values of $\kappa_{1}, \kappa_{2}$ we find the following cases:

- When $\kappa_{1} \neq 0$ and $\kappa_{2} \neq 0$, the three equations (4.12) (or (4.7) which for $\kappa_{1} \neq 0$ are equivalent to them) are three such independent equations; the three equations (4.13) (or (4.8) which for $\kappa_{2} \neq 0$ are equivalent to them) are another choice of three such independent equations. All trigonometry follows from either (4.12) or (4.13).

- When $\kappa_{1}=0$ but $\kappa_{2} \neq 0$, the three equations (4.13) reduce to a single independent equation (4.18). The three equations (4.12) are independent, and (4.7) reduce to trivial identities. All the trigonometry in this case follows from the three equations (4.12).

- When $\kappa_{1} \neq 0$ but $\kappa_{2}=0$, the three equations (4.12) reduce to a single independent equation (4.21). The three equations (4.13) are independent, and (4.8) reduce to trivial identities. All the trigonometry in this case follows from the three equations (4.13).

- When $\kappa_{1}=\kappa_{2}=0$, the three equations (4.12) reduce to a single independent equation, and (4.7) are trivial identities. The three equations (4.13) reduce to a single independent equation, and (4.8) are trivial identities. The trigonometry follows from (4.12), (4.13) and (4.9) which in this case are three independent equations (4.22).

Proof. We consider the generic case with $\kappa_{1} \neq 0, \kappa_{2} \neq 0$, and the cosine theorems (4.7) as the three initial equations (they are independent a fortiori since each of them involves one different angle). Let us obtain the sine theorem (4.9); on the one hand, we compute:

$$
S_{\kappa_{1}}^{2}(a) S_{\kappa_{2}}^{2}(B)=S_{\kappa_{1}}^{2}(a) \frac{\left(1-C_{\kappa_{2}}^{2}(B)\right)}{\kappa_{2}}=\frac{S_{\kappa_{1}}^{2}(a)}{\kappa_{2}}\left(1-\frac{\left(C_{\kappa_{1}}(b)-C_{\kappa_{1}}(a) C_{\kappa_{1}}(c)\right)^{2}}{\kappa_{1}^{2} S_{\kappa_{1}}^{2}(a) S_{\kappa_{1}}^{2}(c)}\right)
$$


where we have introduced the equation $1 \mathrm{~b}$ of (4.7); by expanding and writing the sines in terms of cosines in the numerator, we find:

$$
S_{\kappa_{1}}^{2}(a) S_{\kappa_{2}}^{2}(B)=\frac{1-C_{\kappa_{1}}^{2}(a)-C_{\kappa_{1}}^{2}(b)-C_{\kappa_{1}}^{2}(c)+2 C_{\kappa_{1}}(a) C_{\kappa_{1}}(b) C_{\kappa_{1}}(c)}{\kappa_{1}^{2} \kappa_{2} S_{\kappa_{1}}^{2}(c)} .
$$

(Note that the numerator is the determinant of the Gramm matrix whose elements are the scalar products, in the linear ambient space of the vector model, of the vectors corresponding to the vertices). On the other hand, similar computations show that $S_{\kappa_{1}}^{2}(b) S_{\kappa_{2}}^{2}(A)$ gives again the r.h.s. of (4.24), so that $S_{\kappa_{1}}(a) S_{\kappa_{2}}(B)=S_{\kappa_{1}}(b) S_{\kappa_{2}}(A)$. Likewise, we can prove that $S_{\kappa_{1}}(a) S_{\kappa_{2}}(C)=S_{\kappa_{1}}(c) S_{\kappa_{2}}(A)$, completing the sine theorem. Next, we deduce the dual cosine theorems (4.8). By taking into account the sine theorem (just proven) and (4.24) it can be checked that

$$
S_{\kappa_{2}}(A) S_{\kappa_{2}}(B)=\frac{S_{\kappa_{1}}^{2}(a) S_{\kappa_{2}}^{2}(B)}{S_{\kappa_{1}}(a) S_{\kappa_{1}}(b)}=\frac{1-C_{\kappa_{1}}^{2}(a)-C_{\kappa_{1}}^{2}(b)-C_{\kappa_{1}}^{2}(c)+2 C_{\kappa_{1}}(a) C_{\kappa_{1}}(b) C_{\kappa_{1}}(c)}{\kappa_{1}^{2} \kappa_{2} S_{\kappa_{1}}^{2}(c) S_{\kappa_{1}}(a) S_{\kappa_{1}}(b)} .
$$

The same expression can be obtained starting from

$$
\frac{C_{\kappa_{2}}(C)-C_{\kappa_{2}}(A) C_{\kappa_{2}}(B)}{\kappa_{2} C_{\kappa_{1}}(c)}
$$

and using the three equations (4.7) in order to get rid of the angles, thus writing everything in terms of the sides. Therefore the equation $1 \mathrm{C}$ of (4.8) follows; the two remaining dual cosine theorems can be obtained in the same way. Once we have obtained (4.9) and (4.8) starting from (4.7), the 12 'addition' formulas for sides and angles (with tags $3 \mathrm{aB}, 3 \mathrm{Ab}$, etc.) and the self-dual equations (with tags $4 A B \equiv 4 a b$, etc.) can be straightforwardly deduced from the former theorems. By duality, it is clear that we can start from the cosine theorem for angles.

For the contracted case with $\kappa_{1}=0$ and $\kappa_{2} \neq 0$, as well as for its dual with $\kappa_{2}=0$ and $\kappa_{1} \neq 0$, the proof follows similar steps as in the generic case and we omit it. Finally, the proof for the last case with $\kappa_{1}=\kappa_{2}=0$ is trivial.

We display in table 2 the cosine, dual cosine and sine theorems for each of the nine CK geometries according to the values of the curvatures $\left(\kappa_{1}, \kappa_{2}\right)$. We recall that when $\kappa_{1}$ (resp. $\kappa_{2}$ ) is different from zero, by a suitable choice of length unit (resp. angle unit), it can be reduced to either +1 or -1 : in this table we write the theorems with such an adapted 'natural' choice of units.

\subsection{Relation with the usual approach and with absolute trigonometry}

While the equations we have obtained hold for all the nine 2D CK geometries, the spaces whose trigonometry is well known are the three riemannian spaces of constant curvature - the sphere, the euclidean plane and the hyperbolic plane- which are the members of the CK family with $\kappa_{2}>0$. In these three spaces the usual 'natural' choice of angle units corresponds to making $\kappa_{2}=1$. Therefore by setting $\kappa_{1}=\kappa$ and $\kappa_{2}=1$, our generic relations give rise to the equations of the trigonometry of the three riemannian spaces of constant curvature $\kappa$, and give directly the so-called 'absolute' form of trigonometry, which is valid for the three spaces simultaneously $[3,7,8]$. 
Table 2: Cosine, sine and dual cosine theorems for the nine CK spaces.

\begin{tabular}{|c|c|c|}
\hline $\begin{array}{l}\text { Elliptic }(+1,+1) \\
\quad S O(3) / S O(2)\end{array}$ & $\begin{array}{l}\text { Euclidean }(0,+1) \\
I S O(2) / S O(2)\end{array}$ & $\begin{array}{c}\text { Hyperbolic }(-1,+1) \\
S O(2,1) / S O(2)\end{array}$ \\
\hline $\begin{array}{c}\cos a=\cos b \cos c-\sin b \sin c \cos A \\
\cos b=\cos a \cos c+\sin a \sin c \cos B \\
\cos c=\cos a \cos b+\sin a \sin b \cos C \\
\frac{\sin a}{\sin A}=\frac{\sin b}{\sin B}=\frac{\sin c}{\sin C}\end{array}$ & $\begin{array}{l}a^{2}=b^{2}+c^{2}+2 b c \cos A \\
b^{2}=a^{2}+c^{2}-2 a c \cos B \\
c^{2}=a^{2}+b^{2}-2 a b \cos C \\
\frac{a}{\sin A}=\frac{b}{\sin B}=\frac{c}{\sin C}\end{array}$ & $\begin{aligned} \cosh a= & \cosh b \cosh c+\sinh b \sinh c \cos A \\
\cosh b= & \cosh a \cosh c-\sinh a \sinh c \cos B \\
\cosh c= & \cosh a \cosh b-\sinh a \sinh b \cos C \\
& \frac{\sinh a}{\sin A}=\frac{\sinh b}{\sin B}=\frac{\sinh c}{\sin C}\end{aligned}$ \\
\hline $\begin{array}{l}\cos A=\cos B \cos C-\sin B \sin C \cos a \\
\cos B=\cos A \cos C+\sin A \sin C \cos b \\
\cos C=\cos A \cos B+\sin A \sin B \cos C\end{array}$ & $\begin{array}{l}A=B+C \\
B=A-C \\
C=A-B\end{array}$ & $\begin{array}{l}\cos A=\cos B \cos C-\sin B \sin C \cosh a \\
\cos B=\cos A \cos C+\sin A \sin C \cosh b \\
\cos C=\cos A \cos B+\sin A \sin B \cosh c\end{array}$ \\
\hline $\begin{array}{cc}\text { Co-Euclidean } & (+1,0) \\
\text { Oscillating NH } & I S O(2) / \mathbb{R}\end{array}$ & $\begin{array}{l}\text { Galilean }(0,0) \\
I I S O(1) / \mathbb{R}\end{array}$ & $\begin{array}{l}\text { Co-Minkowskian }(-1,0) \\
\text { Expanding NH } I S O(1,1) / \mathbb{R}\end{array}$ \\
\hline $\begin{array}{c}a=b+c \\
b=a-c \\
c=a-b \\
\frac{\sin a}{A}=\frac{\sin b}{B}=\frac{\sin c}{C}\end{array}$ & $\begin{aligned} a & =b+c \\
b & =a-c \\
c & =a-b \\
\frac{a}{A} & =\frac{b}{B}=\frac{c}{C}\end{aligned}$ & $\begin{array}{c}a=b+c \\
b=a-c \\
c=a-b \\
\frac{\sinh a}{A}=\frac{\sinh b}{B}=\frac{\sinh c}{C}\end{array}$ \\
\hline $\begin{array}{l}A^{2}=B^{2}+C^{2}+2 B C \cos a \\
B^{2}=A^{2}+C^{2}-2 A C \cos b \\
C^{2}=A^{2}+B^{2}-2 A B \cos c\end{array}$ & $\begin{array}{l}A=B+C \\
B=A-C \\
C=A-B\end{array}$ & $\begin{array}{l}A^{2}=B^{2}+C^{2}+2 B C \cosh a \\
B^{2}=A^{2}+C^{2}-2 A C \cosh b \\
C^{2}=A^{2}+B^{2}-2 A B \cosh c\end{array}$ \\
\hline $\begin{array}{l}\text { Co-Hyperbolic }(+1,-1) \\
\text { Anti-de Sitter } S O(2,1) / S O(1,1)\end{array}$ & $\begin{array}{l}\text { Minkowskian }(0,-1) \\
\operatorname{ISO}(1,1) / S O(1,1)\end{array}$ & $\begin{array}{l}\text { Doubly Hyperbolic }(-1,-1) \\
\text { De Sitter } S O(2,1) / S O(1,1)\end{array}$ \\
\hline $\begin{array}{c}\cos a=\cos b \cos c-\sin b \sin c \cosh A \\
\cos b=\cos a \cos c+\sin a \sin c \cosh B \\
\cos c=\cos a \cos b+\sin a \sin b \cosh C \\
\frac{\sin a}{\sinh A}=\frac{\sin b}{\sinh B}=\frac{\sin c}{\sinh C}\end{array}$ & $\begin{array}{l}a^{2}=b^{2}+c^{2}+2 b c \cosh A \\
b^{2}=a^{2}+c^{2}-2 a c \cosh B \\
c^{2}=a^{2}+b^{2}-2 a b \cosh C \\
\frac{a}{\sinh A}=\frac{b}{\sinh B}=\frac{c}{\sinh C}\end{array}$ & $\begin{array}{c}\cosh a=\cosh b \cosh c+\sinh b \sinh c \cosh A \\
\cosh b=\cosh a \cosh c-\sinh a \sinh c \cosh B \\
\cosh c=\cosh a \cosh b-\sinh a \sinh b \cosh C \\
\frac{\sinh a}{\sinh A}=\frac{\sinh b}{\sinh B}=\frac{\sinh c}{\sinh C}\end{array}$ \\
\hline $\begin{array}{l}\cosh A=\cosh B \cosh C+\sinh B \sinh C \cos a \\
\cosh B=\cosh A \cosh C-\sinh A \sinh C \cos b \\
\cosh C=\cosh A \cosh B-\sinh A \sinh B \cos c\end{array}$ & $\begin{array}{l}A=B+C \\
B=A-C \\
C=A-B\end{array}$ & $\begin{array}{l}\cosh A=\cosh B \cosh C+\sinh B \sinh C \cosh a \\
\cosh B=\cosh A \cosh C-\sinh A \sinh C \cosh b \\
\cosh C=\cosh A \cosh B-\sinh A \sinh B \cosh c\end{array}$ \\
\hline
\end{tabular}

In this connection an elementary but relevant point should be kept in mind: spherical, euclidean and hyperbolic trigonometry is usually formulated in terms of the inner angles. The natural angles when the triangle is seen as a line loop are however not the three inner angles; one of them must be an external angle, like our $A$. When $\kappa_{2}=1$, the measure of a straight angle is equal to $\pi$, and the three internal angles $\alpha, \beta, \gamma$ are related to our $A, B$, $C$ as $\alpha=\pi-A, \beta=B, \gamma=C$, so that the triangle angular excess $\Delta$, usually defined as $\alpha+\beta+\gamma-\pi$ appears here as $-A+B+C$, and thus it does not involve $\pi$. The quantity we have denoted (see (4.16))

$$
P-A=\frac{\Delta}{2}=\frac{-A+B+C}{2}=\frac{\alpha+\beta+\gamma}{2}-\frac{\pi}{2}
$$

differs by $\pi / 2$ from the half sum of internal angles, so our sines and cosines of $P-A$ appear as cosines and sines of the half sum of internal angles. These elementary facts account for all apparent discrepancies between the particularization of the general equations given in this paper and the ones found in the literature for the three constant curvature riemannian spaces (for instance, $[34,35,36]$ ). The use of the three inner angles makes $\pi$ to enter unavoidably in the angle sum, the definition of angular excess, the Gauss-Bonnet theorem 
for triangles, etc., and thus seems to preclude analogous relations in the cases with a locally lorentzian metric, where $\pi$ does not properly belong anymore. Incidentaly, in the three riemannian geometries any angle can be taken as external, but there is no longer such a freedom in the cases $\kappa_{2} \leq 0$, where the choice of the external angle $A$ in any first-kind loop is dictated by the geometry itself, and is always opposite to the largest side $a$.

Thus while the choice for angles in this paper can be consistently done in all the nine CK spaces and affords the equations in a natural form, it also introduces some minus signs which are absent in the trigonometry of the three riemannian spaces in their standard formulation with internal angles. Without any further reason (as the inclusion of the nine geometries provides) it would seem unwise to modify the time-honoured form these equations have in the three familiar constant curvature spaces; however this modification is an essential step to make the equations meaningful for the nine cases. This way the approach is more general than absolute trigonometry, because it applies to the whole set of nine CK spaces, and the explicit presence of $\kappa_{2}$ allows the consideration of the scheme within a complete duality, which otherwise would be hidden, showing up only in the spherical case. For instance, the dual of the hyperbolic geometry is the anti-de Sitter one, whose natural metric is lorentzian; simultaneous consideration of riemannian and pseudoriemannian cases is therefore essential to fully display duality.

When put in the right perspective, the possibility of formulating trigonometry for the nine CK spaces in a single unified way where all equations are analogous and directly meaningful in all cases is almost a triviality. But to unfold this view we have to abandon, by making it first explicit, the implicit restriction $\kappa_{2}=1$ which amounts to measuring angles in radians and is universally enforced for the three riemannian cases. While the curvature $\kappa_{1}$ allows to explicitly distinguishing between the sphere, euclidean and hyperbolic plane in the absolute form of the trigonometric equations, the 'dual' constant $\kappa_{2}$ is restricted to a single particular (positive) value, so it cannot be given the attention it deserves when it is allowed to take on any real value. And further, the usual definition of twopoint homogeneity [22] excludes the degenerate riemannian and pseudoriemannian spaces (where the isotropy subgroup is not compact, and its action on the 'unit sphere' is not transitive). For instance the paper by Hsiang [3] is explicitly addressed to the study of trigonometry of rank-one spaces, and covers in a single run the three riemannian constant curvature spaces; however no reference to the pseudoriemannian or degenerate riemannian planes is ever made, nor it is felt as missing in that paper, because the restriction to twopoint homogeneous spaces precludes this consideration from the beginning. In some cases even a slightly non-standard definition of spaces of constant curvature is made [37] so as to embrace only the spaces whose isotropy subgroup is compact (i.e, $\kappa_{2}>0$ ).

An interesting relation between pairs of homogeneous symmetric spaces is Cartan duality; it relates spherical and hyperbolic geometry and is ultimately responsible for the fact that hyperbolic geometry holds on the sphere of 'imaginary' radius; this was historically recognized by Lambert before the Lobachewski hyperbolic geometry was satisfactorily settled, and was considered by Lobachewski himself as an unconclusive evidence supporting the idea that his geometry was free of contradictions. Each Cartan duality is related to an involutive automorphism of the Lie algebra giving rise to a non-compact Lie algebra if applied to a compact one. Our approach is based on two commuting involutive automorphisms, so it contains two Cartan dualities; each is implemented by the change of sign in one of the two constants $\kappa_{1}, \kappa_{2}$. In this sense, the hyperbolic space is Cartan dual to the sphere, and also Cartan dual to the de Sitter sphere. 


\subsection{A compact notation}

In order not to burden the discussion with permanent reference to the sign differences related to the side $a$ and angle $A$ (which are unavoidable if one wants a formulation valid in all nine cases), the introduction of an auxiliary notation turns out to be extremely convenient. We will denote the three sides as $x_{i}, i=1,2,3$ and the three angles as $X_{I}$, $I=1,2,3$ according to

$$
x_{1}=-a \quad x_{2}=b \quad x_{3}=c \quad X_{1}=-A \quad X_{2}=B \quad X_{3}=C
$$

(note the built-in minus sign in $x_{1}$ and $X_{1}$, which is natural when the triangle is considered as a point loop with the side $a$ traversed backwards, or as a side loop with the angle $A$ rotated backwards). With this notation, the basic equation (3.26) based in the vertex 2 is

$$
e^{x_{1} P} e^{X_{3} J} e^{x_{2} P} e^{X_{1} J} e^{x_{3} P} e^{X_{2} J}=1
$$

and their alternative equivalent versions based in the vertex $j$ can be written as:

$$
e^{x_{i} P} e^{X_{K} J} e^{x_{j} P} e^{X_{I} J} e^{x_{k} P} e^{X_{J} J}=1
$$

for any cyclic permutation $i=I, j=J, k=K$ of the three indices 123 . In what follows we will always adopt this convention which makes all equations of trigonometry explicitly invariant under any cyclic permutation of the 'oriented' sides $x_{i}$ and angles $X_{I}$; the use of capital indices will also help to distinguish between lengths and angles.

The triangular loop lateral excess $\delta(3.18)$ and angular excess $\Delta$ (3.16) appear in the present notation as the symmetric sums of the three 'oriented' sides or angles as:

$$
\delta=x_{1}+x_{2}+x_{3}=-a+b+c \quad \Delta=X_{1}+X_{2}+X_{3}=-A+B+C .
$$

It will be also convenient to replace the excesses by the quantities

$$
e:=\delta / 2 \quad E:=\Delta / 2
$$

and to introduce three other quantities as well as their three duals:

$$
e_{i}:=x_{i}-e \quad E_{I}:=X_{I}-E .
$$

They verify the following equations

$$
\begin{array}{llll}
e_{i}-e_{j}=x_{i}-x_{j} & e_{i}+e_{j}=-x_{k} & e+e_{i}=x_{i} & e-e_{i}=x_{j}+x_{k} \\
E_{I}-E_{J}=X_{I}-X_{J} & E_{I}+E_{J}=-X_{K} & E+E_{I}=X_{I} & E-E_{I}=X_{J}+X_{K}
\end{array}
$$

and are related with the half sums $p$ and $P(4.14)$ by:

$$
\begin{array}{llll}
e=p-a & e_{1}=-p & e_{2}=p-c & e_{3}=p-b \\
E=P-A & E_{1}=-P & E_{2}=P-C & E_{3}=P-B
\end{array}
$$

Note that $e_{1}$ is different from zero and negative, while $e_{2}, e_{3}$ are (generically) different from zero and positive, just like the three sides $x_{1}$ and $x_{2}, x_{3}$. The same holds for the quantities related to angular excesses: $E_{1}$ is different from zero and negative, while $E_{2}, E_{3}$ are (generically) different from zero and positive. If $\kappa_{2}=0$ then the three $e_{i}$ reduce to 
the sides $x_{i}(\delta=e=0)$. Dually, if $\kappa_{1}=0$ then the three $E_{I}$ reduce to the angles $X_{I}$ $(\Delta=E=0)$.

In terms of these quantities, the equations of trigonometry we have obtained, including the alternative forms for the cosine theorems (4.15) and (4.16), turn out to be

$$
\begin{array}{ll}
\text { 1i } & C_{\kappa_{1}}\left(x_{i}\right)=C_{\kappa_{1}}\left(x_{j}\right) C_{\kappa_{1}}\left(x_{k}\right)-\kappa_{1} S_{\kappa_{1}}\left(x_{j}\right) S_{\kappa_{1}}\left(x_{k}\right) C_{\kappa_{2}}\left(X_{I}\right) \\
\text { 1I } & C_{\kappa_{2}}\left(X_{I}\right)=C_{\kappa_{2}}\left(X_{J}\right) C_{\kappa_{2}}\left(X_{K}\right)-\kappa_{2} S_{\kappa_{2}}\left(X_{J}\right) S_{\kappa_{2}}\left(X_{K}\right) C_{\kappa_{1}}\left(x_{i}\right) \\
1 " \mathrm{i} & 2 S_{\kappa_{1}}(e) S_{\kappa_{1}}\left(e_{i}\right)=-\kappa_{2} S_{\kappa_{1}}\left(x_{j}\right) S_{\kappa_{1}}\left(x_{k}\right) V_{\kappa_{2}}\left(X_{I}\right) \\
1 " \mathrm{I} & 2 S_{\kappa_{2}}(E) S_{\kappa_{2}}\left(E_{I}\right)=-\kappa_{1} S_{\kappa_{2}}\left(X_{J}\right) S_{\kappa_{2}}\left(X_{K}\right) V_{\kappa_{1}}\left(x_{i}\right) \\
2 & \frac{S_{\kappa_{1}}\left(x_{i}\right)}{S_{\kappa_{2}}\left(X_{I}\right)}=\frac{S_{\kappa_{1}}\left(x_{j}\right)}{S_{\kappa_{2}}\left(X_{J}\right)}=\frac{S_{\kappa_{1}}\left(x_{k}\right)}{S_{\kappa_{2}}\left(X_{K}\right)} \\
\text { 3iJ } & S_{\kappa_{1}}\left(x_{i}\right) C_{\kappa_{2}}\left(X_{J}\right)=-C_{\kappa_{1}}\left(x_{j}\right) S_{\kappa_{1}}\left(x_{k}\right)-S_{\kappa_{1}}\left(x_{j}\right) C_{\kappa_{1}}\left(x_{k}\right) C_{\kappa_{2}}\left(X_{I}\right) \\
\text { 3Ij } & S_{\kappa_{2}}\left(X_{I}\right) C_{\kappa_{1}}\left(x_{j}\right)=-C_{\kappa_{2}}\left(X_{J}\right) S_{\kappa_{2}}\left(X_{K}\right)-S_{\kappa_{2}}\left(X_{J}\right) C_{\kappa_{2}}\left(X_{K}\right) C_{\kappa_{1}}\left(x_{i}\right) \\
\text { 4IJ } \equiv 4 \mathrm{ij} & \kappa_{2} S_{\kappa_{2}}\left(X_{I}\right) S_{\kappa_{2}}\left(X_{J}\right)-C_{\kappa_{2}}\left(X_{I}\right) C_{\kappa_{2}}\left(X_{J}\right) C_{\kappa_{1}}\left(x_{k}\right) \\
& =\kappa_{1} S_{\kappa_{1}}\left(x_{i}\right) S_{\kappa_{1}}\left(x_{j}\right)-C_{\kappa_{1}}\left(x_{i}\right) C_{\kappa_{1}}\left(x_{j}\right) C_{\kappa_{2}}\left(X_{K}\right)
\end{array}
$$

and we now have a completely uniform pattern, without casual signs. When $\kappa_{2}=0$, the three equations 1"i clearly imply $S_{\kappa_{1}}(e)=0$, as already commented. This means that when the constant $\kappa_{2} \rightarrow 0$, the sine of the lateral excess of the triangle also goes to zero, but the quotient $S_{\kappa_{1}}(e) / \kappa_{2}$ remains finite, and is given by:

$$
\frac{S_{\kappa_{1}}(e)}{\kappa_{2}}=-\frac{S_{\kappa_{1}}\left(x_{j}\right) S_{\kappa_{1}}\left(x_{k}\right) V_{\kappa_{2}}\left(X_{I}\right)}{2 S_{\kappa_{1}}\left(e_{i}\right)}
$$

Dually, when $\kappa_{1}=0$, the equations 1"I lead to $S_{\kappa_{2}}(E)=0$; the sine of the angular excess of the triangle goes to zero when $\kappa_{1} \rightarrow 0$, but the quotient $S_{\kappa_{2}}(E) / \kappa_{1}$ remains finite:

$$
\frac{S_{\kappa_{2}}(E)}{\kappa_{1}}=-\frac{S_{\kappa_{2}}\left(X_{J}\right) S_{\kappa_{2}}\left(X_{K}\right) V_{\kappa_{1}}\left(x_{i}\right)}{2 S_{\kappa_{2}}\left(E_{I}\right)}
$$

\subsection{Area and coarea and the dualities length/area and angle/coarea}

The previous expressions have shown the natural appearance in this group theoretical approach of the combinations $S_{\kappa_{1}}(e) / \kappa_{2}$ and $S_{\kappa_{2}}(E) / \kappa_{1}$ which are well defined in all the nine cases, no matter of the values of $\kappa_{1}$ and $\kappa_{2}$. This is because $S_{\kappa_{1}}(e)$ goes to zero linearly with the 'line curvature' $\kappa_{2}$, and therefore has a kind of residue with a well defined value even in the (limiting) case $\kappa_{2}=0$. Dually, the same phenomenon happens for $S_{\kappa_{2}}(E)$, which vanishes when $\kappa_{1}=0$.

By construction, the angular excess of a triangle loop is additive under decomposition of a triangle loop into two. Thus it is obvious that $S_{\kappa_{2}}(E) / \kappa_{1}$ is related to the triangular loop area. This relation is very well known in the two riemannian spherical and hyperbolic geometries, where the standard expression for the absolute value $\mathcal{S}$ of the area enclosed by the triangle loop is easy to derive from the Gauss-Bonnet theorem and is related to the angular excess by $\kappa_{1} \mathcal{S}=\Delta$. This suggests a purely group theoretical definition of area (and its dual quantity, coarea $s$ ) for triangle loops, which extends the earlier group theoretical definitions of length and angle. This group theoretical definition holds for all the nine 2D CK spaces, no matter of the values of $\kappa_{1}$ or $\kappa_{2}$, hence applying also to the pseudoriemannian and degenerate riemannian CK spaces. This should be in full agreement 
with the standard definition for area based on differential geometry. All these requirements are satisfied by the following definitions of area and coarea for a triangle:

$$
\mathcal{S}:=\frac{\Delta}{\kappa_{1}} \quad s:=\frac{\delta}{\kappa_{2}}
$$

All appearances of $S_{\kappa_{2}}(E) / \kappa_{1}$ in the equations of trigonometry could be rewritten in terms of trigonometric functions of the area of the loop, and dually for $S_{\kappa_{1}}(e) / \kappa_{2}$ and coarea. In this rewriting, the label naturally associated to the area is $\kappa_{1}^{2} \kappa_{2}$, while the coarea label is $\kappa_{1} \kappa_{2}^{2}$; this makes sense as area should be to the product $P_{1} P_{2}$ (resp. coarea to $J_{12} P_{2}$ ) what length is to $P_{1}$ with label $\kappa_{1}$ and angle to $J_{12}$ with label $\kappa_{2}$. For the two basic sine and cosine functions of area and coarea we have:

$$
\begin{aligned}
& C_{\kappa_{1}^{2} \kappa_{2}}(\mathcal{S}):=C_{\kappa_{2}}(\Delta)=C_{\kappa_{2}}(2 E) \quad S_{\kappa_{1}^{2} \kappa_{2}}(\mathcal{S}):=\frac{S_{\kappa_{2}}(\Delta)}{\kappa_{1}}=\frac{S_{\kappa_{2}}(2 E)}{\kappa_{1}} \\
& C_{\kappa_{1} \kappa_{2}^{2}}(s):=C_{\kappa_{1}}(\delta)=C_{\kappa_{1}}(2 e) \quad S_{\kappa_{1} \kappa_{2}^{2}}(s):=\frac{S_{\kappa_{1}}(\delta)}{\kappa_{2}}=\frac{S_{\kappa_{1}}(2 e)}{\kappa_{2}} .
\end{aligned}
$$

Therefore the two quotients (4.38) and (4.37) are the sine of half the area and half the coarea of the triangle loop, each with its canonical label:

$$
\frac{S_{\kappa_{2}}(E)}{\kappa_{1}}=S_{\kappa_{1}^{2} \kappa_{2}}(\mathcal{S} / 2) \quad \frac{S_{\kappa_{1}}(e)}{\kappa_{2}}=S_{\kappa_{1} \kappa_{2}^{2}}(s / 2) .
$$

A length/area and angle/coarea dualities for the sphere have been recently discussed by Arnol'd [24] in a paper devoted to the geometry of spherical curves. These 'dualities' are indeed a general property for all the nine CK geometries, and follow directly from the fundamental self-duality of the whole scheme of CK spaces (between lengths and angles), together with the 'transference' from angles $E$ (or $\Delta$ ) to areas $\mathcal{S} / 2$ (or from lengths $e$ (or $\delta$ ) to coareas $s / 2$ ) implicitly contained in the equations (4.41). However, while these dualities are present in all CK geometries, they are only clearly visible for the sphere, where by a suitable choice of length and angle units the two constants $\kappa_{1}$ and $\kappa_{2}$ can be reduced to 1. In this spherical case the labels of either length, angle, area or coarea are all equal, so the transference from angle to area (or length to coarea) amounts to a simple equality between numerical values, therefore displaying in a single geometry the full richness of the CK scheme. In this case all trigonometrical quantities (either lengths, angles, areas or coareas) appear in all trigonometric formulas as arguments of circular trigonometric functions.

In other CK geometries where some of the constants are negative, unveiling these dualities requires explicit use of a transference similar to those in (4.41). The leading idea in Arnol'd paper is to link to each spherical curve its 'dual' and its 'derivative' curves determined from the given curve by moving a quadrant along the normal or the tangent to the curve. This triple has very interesting properties, giving rise to a kind of triality, and it is within its study that the length/area and angle/coarea dualities appear. This spherical triality must have an interesting 'hyperbolic' version where the three related curves - the given one, its dual and its derivative - lives in a different space, say the hyperbolic plane, the co-hyperbolic or anti-de Sitter sphere and the douby hyperbolic or de Sitter sphere. This triality seems worth studying, mainly in view of the strong current interest in the anti-de Sitter spacetime and should be related to the conformal field theory on the sphere at infinity in hyperbolical geometry. 


\subsection{The trigonometric equations in the minimal form}

By adding the two new triangular loop quantities, area $\mathcal{S}$ and coarea $s$, to sides and angles, all the basic equations can be written in a minimal form, with no explicit constants $\kappa_{1}, \kappa_{2}$. These equations (as well as all equations derived from them), are directly meaningful for all the nine cases and do not reduce to trivial identities. Furthermore the versed sine of the angle $X_{I}$ in (4.37) and side $x_{i}$ in (4.38) can be expressed in terms of the sine of half the angle or the side using the relation (A.6). Thus the equations read

$$
\begin{array}{ll}
1 " \mathrm{i} & S_{\kappa_{1} \kappa_{2}^{2}}(s / 2) S_{\kappa_{1}}\left(e_{i}\right)=-S_{\kappa_{1}}\left(x_{j}\right) S_{\kappa_{1}}\left(x_{k}\right) S_{\kappa_{2}}^{2}\left(X_{I} / 2\right) \\
1 " \mathrm{I} & S_{\kappa_{1}^{2} \kappa_{2}}(\mathcal{S} / 2) S_{\kappa_{2}}\left(E_{I}\right)=-S_{\kappa_{2}}\left(X_{J}\right) S_{\kappa_{2}}\left(X_{K}\right) S_{\kappa_{1}}^{2}\left(x_{i} / 2\right) \\
2 & \frac{S_{\kappa_{1}}\left(x_{i}\right)}{S_{\kappa_{2}}\left(X_{I}\right)}=\frac{S_{\kappa_{1}}\left(x_{j}\right)}{S_{\kappa_{2}}\left(X_{J}\right)}=\frac{S_{\kappa_{1}}\left(x_{k}\right)}{S_{\kappa_{2}}\left(X_{K}\right)} .
\end{array}
$$

Two things are worth remarking. First, the corresponding equations for any two particular spaces only differ by the implicit appearances of the constants (curvatures) $\kappa_{1}, \kappa_{2}$ (and also $\kappa_{1}^{2} \kappa_{2}, \kappa_{1} \kappa_{2}^{2}$ ) as labels of the trigonometric functions of sides, angles (and also area, coarea) respectively. This is reminiscent to the minimal coupling idea in general relativity: no explicitly dependent curvature terms should be introduced in the basic free equations when formulating the corresponding equation for a curved spacetime, but only those introduced (implicitly) through the conexion. In (4.42), any trigonometric equation in a space with curvatures $\kappa_{1}, \kappa_{2}$ different from zero can be obtained from the corresponding flat one by introducing the corresponding implicit label in the trigonometric functions of sides, angles, area and coarea, but without any explicitly dependent $\kappa_{1}, \kappa_{2}$ term. Secondly, the only trigonometric function involved in these equations is the sine, which reduces to the variable itself when the label equals to zero. Therefore the implementation of this kind of 'minimal coupling' to obtain the general equations (4.42) consists simply in replacing each term entering the 'purely flat' $\kappa_{1}=0, \kappa_{2}=0$ equations

$$
\begin{array}{ll}
1 " \mathrm{i} & (s / 2) e_{i}=-x_{j} x_{k}\left(X_{I} / 2\right)^{2} \\
1 " \mathrm{I} & (\mathcal{S} / 2) E_{I}=-X_{J} X_{K}\left(x_{i} / 2\right)^{2} \\
2 & \frac{x_{i}}{X_{I}}=\frac{x_{j}}{X_{J}}=\frac{x_{k}}{X_{K}}
\end{array}
$$

by their sines, each with the corresponding label.

\section{A trigonometric bestiarium}

Starting from the set of basic equations (4.42), we can easily derive a complete trigonometric bestiarium. All equations are written in a way which is simultaneously meaningful for all the nine geometries, and by allowing area and coarea to enter the basic equations no explicit constants $\kappa_{1}, \kappa_{2}$ ever appear. Should area and coarea be avoided in favour of $E$ or $e$, then all explicit appearances of $\kappa_{1}, \kappa_{2}$ can be reduced (see (4.41)) to the combinations $S_{\kappa_{1}}(e) / \kappa_{2}$ or $S_{\kappa_{2}}(E) / \kappa_{1}$, where the joint appearance of both curvatures $\kappa_{1}$ and $\kappa_{2}$ (one as a label and the other explicitly) reminds that the length $e$ is not directly a distance between two points or the angle $E$ is not an angle between two lines, but both come from the triangle as a whole.

The minus signs explicitly appearing in (4.42), and in most of the ensuing equations are artifacts following our definitions on $x_{i}, e_{i}$ and $X_{I}, E_{I}$. These signs will disappear when 
the equations are rewritten in terms of the positive quantities $a, b, c, p, s$ and $A, B, C, P, \mathcal{S}$, but then the casual signs related to the external angle will spoil the uniform appearance of the equations.

\subsection{Equations of Euler, Gauss-Delambre-Mollweide and Napier}

Equation 1"i of (4.42) gives directly

$$
S_{\kappa_{2}}^{2}\left(\frac{X_{I}}{2}\right)=-\frac{S_{\kappa_{1} \kappa_{2}^{2}}(s / 2) S_{\kappa_{1}}\left(e_{i}\right)}{S_{\kappa_{1}}\left(x_{j}\right) S_{\kappa_{1}}\left(x_{k}\right)} .
$$

Consequently, the corresponding relation for the cosine is derived from it by applying (A.1) and (A.32):

$$
C_{\kappa_{2}}^{2}\left(\frac{X_{I}}{2}\right)=\frac{S_{\kappa_{1}}\left(e_{j}\right) S_{\kappa_{1}}\left(e_{k}\right)}{S_{\kappa_{1}}\left(x_{j}\right) S_{\kappa_{1}}\left(x_{k}\right)}
$$

and the quotient of the above relations gives

$$
T_{\kappa_{2}}^{2}\left(\frac{X_{I}}{2}\right)=-\frac{S_{\kappa_{1} \kappa_{2}^{2}}(s / 2) S_{\kappa_{1}}\left(e_{i}\right)}{S_{\kappa_{1}}\left(e_{j}\right) S_{\kappa_{1}}\left(e_{k}\right)} .
$$

In the same way their duals can be deduced:

$$
\begin{aligned}
& S_{\kappa_{1}}^{2}\left(\frac{x_{i}}{2}\right)=-\frac{S_{\kappa_{1}^{2} \kappa_{2}}(\mathcal{S} / 2) S_{\kappa_{2}}\left(E_{I}\right)}{S_{\kappa_{2}}\left(X_{J}\right) S_{\kappa_{2}}\left(X_{K}\right)} \quad C_{\kappa_{1}}^{2}\left(\frac{x_{i}}{2}\right)=\frac{S_{\kappa_{2}}\left(E_{J}\right) S_{\kappa_{2}}\left(E_{K}\right)}{S_{\kappa_{2}}\left(X_{J}\right) S_{\kappa_{2}}\left(X_{K}\right)} \\
& T_{\kappa_{1}}^{2}\left(\frac{x_{i}}{2}\right)=-\frac{S_{\kappa_{1}^{2} \kappa_{2}}(\mathcal{S} / 2) S_{\kappa_{2}}\left(E_{I}\right)}{S_{\kappa_{2}}\left(E_{J}\right) S_{\kappa_{2}}\left(E_{K}\right)} .
\end{aligned}
$$

These relations were obtained by Euler for the sphere. From them and by using (A.4), expressions for the sine of the angles (and not their half) in terms of the sides can be deduced, as well as for the sides in terms of the angles (dual of the previous ones):

$$
\begin{aligned}
& S_{\kappa_{2}}\left(X_{I}\right)=-\frac{2}{S_{\kappa_{1}}\left(x_{j}\right) S_{\kappa_{1}}\left(x_{k}\right)}\left\{-S_{\kappa_{1} \kappa_{2}^{2}}(s / 2) S_{\kappa_{1}}\left(e_{i}\right) S_{\kappa_{1}}\left(e_{j}\right) S_{\kappa_{1}}\left(e_{k}\right)\right\}^{1 / 2} \\
& S_{\kappa_{1}}\left(x_{i}\right)=-\frac{2}{S_{\kappa_{2}}\left(X_{J}\right) S_{\kappa_{2}}\left(X_{K}\right)}\left\{-S_{\kappa_{1}^{2} \kappa_{2}}(\mathcal{S} / 2) S_{\kappa_{2}}\left(E_{I}\right) S_{\kappa_{2}}\left(E_{J}\right) S_{\kappa_{2}}\left(E_{K}\right)\right\}^{1 / 2} .
\end{aligned}
$$

By dividing both expressions and taking into account the sine theorem we find

$$
\frac{S_{\kappa_{1}}\left(x_{i}\right)}{S_{\kappa_{2}}\left(X_{I}\right)}=\frac{S_{\kappa_{1}}\left(x_{j}\right)}{S_{\kappa_{2}}\left(X_{J}\right)}=\frac{S_{\kappa_{1}}\left(x_{k}\right)}{S_{\kappa_{2}}\left(X_{K}\right)}=\frac{\left\{-S_{\kappa_{1} \kappa_{2}^{2}}(s / 2) S_{\kappa_{1}}\left(e_{i}\right) S_{\kappa_{1}}\left(e_{j}\right) S_{\kappa_{1}}\left(e_{k}\right)\right\}^{1 / 2}}{\left\{-S_{\kappa_{1}^{2} \kappa_{2}}(\mathcal{S} / 2) S_{\kappa_{2}}\left(E_{I}\right) S_{\kappa_{2}}\left(E_{J}\right) S_{\kappa_{2}}\left(E_{K}\right)\right\}^{1 / 2}} .
$$

On the other hand, by combining the formulas (5.1)-(5.3) in different ways we obtain

$$
\begin{aligned}
& C_{\kappa_{2}}\left(\frac{X_{I}}{2}\right) C_{\kappa_{2}}\left(\frac{X_{J}}{2}\right) C_{\kappa_{2}}\left(\frac{X_{K}}{2}\right)=\frac{S_{\kappa_{1}}\left(e_{i}\right) S_{\kappa_{1}}\left(e_{j}\right) S_{\kappa_{1}}\left(e_{k}\right)}{S_{\kappa_{1}}\left(x_{i}\right) S_{\kappa_{1}}\left(x_{j}\right) S_{\kappa_{1}}\left(x_{k}\right)} \\
& S_{\kappa_{2}}\left(\frac{X_{I}}{2}\right) S_{\kappa_{2}}\left(\frac{X_{J}}{2}\right) C_{\kappa_{2}}\left(\frac{X_{K}}{2}\right)=-\frac{S_{\kappa_{1}}\left(e_{i}\right) S_{\kappa_{1}}\left(e_{j}\right) S_{\kappa_{1} \kappa_{2}^{2}}(s / 2)}{S_{\kappa_{1}}\left(x_{i}\right) S_{\kappa_{1}}\left(x_{j}\right) S_{\kappa_{1}}\left(x_{k}\right)}
\end{aligned}
$$




$$
T_{\kappa_{2}}\left(\frac{X_{I}}{2}\right) T_{\kappa_{2}}\left(\frac{X_{J}}{2}\right)=-\frac{S_{\kappa_{1} \kappa_{2}^{2}}(s / 2)}{S_{\kappa_{1}}\left(e_{k}\right)} \quad \frac{T_{\kappa_{2}}\left(\frac{X_{I}}{2}\right)}{T_{\kappa_{2}}\left(\frac{X_{J}}{2}\right)}=\frac{S_{\kappa_{1}}\left(e_{i}\right)}{S_{\kappa_{1}}\left(e_{j}\right)}
$$

and in the same way their duals. Starting from these equations we can deduce the general forms, valid for all the nine geometries, of the Gauss-Delambre-Mollweide analogies. For instance, let us consider the identity (cf. (A.9))

$$
S_{\kappa_{2}}\left(\frac{X_{I}+X_{J}}{2}\right)=S_{\kappa_{2}}\left(\frac{X_{I}}{2}\right) C_{\kappa_{2}}\left(\frac{X_{J}}{2}\right)+S_{\kappa_{2}}\left(\frac{X_{J}}{2}\right) C_{\kappa_{2}}\left(\frac{X_{I}}{2}\right) .
$$

We apply the second relation of (5.7) and group common terms

$$
S_{\kappa_{2}}\left(\frac{X_{I}+X_{J}}{2}\right)=-\frac{S_{\kappa_{1} \kappa_{2}^{2}}(s / 2) S_{\kappa_{1}}\left(e_{k}\right)}{S_{\kappa_{1}}\left(x_{i}\right) S_{\kappa_{1}}\left(x_{j}\right)} \frac{\left(S_{\kappa_{1}}\left(e_{i}\right)+S_{\kappa_{1}}\left(e_{j}\right)\right)}{S_{\kappa_{1}}\left(x_{k}\right) S_{\kappa_{2}}\left(\frac{X_{K}}{2}\right)}
$$

and by introducing the Euler equation (5.1) and using (A.4), (A.14) and (4.34) we find:

$$
S_{\kappa_{2}}\left(\frac{X_{I}+X_{J}}{2}\right)=-\frac{S_{\kappa_{2}}\left(\frac{X_{K}}{2}\right)}{C_{\kappa_{1}}\left(\frac{x_{k}}{2}\right)} C_{\kappa_{1}}\left(\frac{x_{i}-x_{j}}{2}\right) .
$$

Likewise, we can obtain the full set of Gauss-Delambre-Mollweide analogies:

$$
\begin{array}{ll}
\frac{S_{\kappa_{2}}\left(\frac{X_{I}+X_{J}}{2}\right)}{S_{\kappa_{2}}\left(\frac{X_{K}}{2}\right)}=-\frac{C_{\kappa_{1}}\left(\frac{x_{i}-x_{j}}{2}\right)}{C_{\kappa_{1}}\left(\frac{x_{k}}{2}\right)} & \frac{C_{\kappa_{2}}\left(\frac{X_{I}+X_{J}}{2}\right)}{C_{\kappa_{2}}\left(\frac{X_{K}}{2}\right)}=\frac{C_{\kappa_{1}}\left(\frac{x_{i}+x_{j}}{2}\right)}{C_{\kappa_{1}}\left(\frac{x_{k}}{2}\right)} \\
\frac{S_{\kappa_{2}}\left(\frac{X_{I}-X_{J}}{2}\right)}{S_{\kappa_{2}}\left(\frac{X_{K}}{2}\right)}=\frac{S_{\kappa_{1}}\left(\frac{x_{i}-x_{j}}{2}\right)}{S_{\kappa_{1}}\left(\frac{x_{k}}{2}\right)} & \frac{C_{\kappa_{2}}\left(\frac{X_{I}-X_{J}}{2}\right)}{C_{\kappa_{2}}\left(\frac{X_{K}}{2}\right)}=-\frac{S_{\kappa_{1}}\left(\frac{x_{i}+x_{j}}{2}\right)}{S_{\kappa_{1}}\left(\frac{x_{k}}{2}\right)} .
\end{array}
$$

By quotient of these expressions we obtain the Napier analogies:

$$
\begin{array}{ll}
\frac{T_{\kappa_{2}}\left(\frac{X_{I}+X_{J}}{2}\right)}{T_{\kappa_{2}}\left(\frac{X_{K}}{2}\right)}=-\frac{C_{\kappa_{1}}\left(\frac{x_{i}-x_{j}}{2}\right)}{C_{\kappa_{1}}\left(\frac{x_{i}+x_{j}}{2}\right)} & \frac{T_{\kappa_{2}}\left(\frac{X_{I}-X_{J}}{2}\right)}{T_{\kappa_{2}}\left(\frac{X_{K}}{2}\right)}=-\frac{S_{\kappa_{1}}\left(\frac{x_{i}-x_{j}}{2}\right)}{S_{\kappa_{1}}\left(\frac{x_{i}+x_{j}}{2}\right)} \\
\frac{T_{\kappa_{1}}\left(\frac{x_{i}+x_{j}}{2}\right)}{T_{\kappa_{1}}\left(\frac{x_{k}}{2}\right)}=-\frac{C_{\kappa_{2}}\left(\frac{X_{I}-X_{J}}{2}\right)}{C_{\kappa_{2}}\left(\frac{X_{I}+X_{J}}{2}\right)} & \frac{T_{\kappa_{1}}\left(\frac{x_{i}-x_{j}}{2}\right)}{T_{\kappa_{1}}\left(\frac{x_{k}}{2}\right)}=-\frac{S_{\kappa_{2}}\left(\frac{X_{I}-X_{J}}{2}\right)}{S_{\kappa_{2}}\left(\frac{X_{I}+X_{J}}{2}\right)}
\end{array}
$$

and by further division, we obtain the self-dual equations:

$$
\frac{T_{\kappa_{2}}\left(\frac{X_{I}+X_{J}}{2}\right)}{T_{\kappa_{2}}\left(\frac{X_{I}-X_{J}}{2}\right)}=\frac{T_{\kappa_{1}}\left(\frac{x_{i}+x_{j}}{2}\right)}{T_{\kappa_{1}}\left(\frac{x_{i}-x_{j}}{2}\right)} .
$$

These relations cover the general versions of practically every equation found in textbooks on spherical or hyperbolic trigonometry. Others, which we refrain from writing, are very easily obtained from them. 


\subsection{Equations for area and coarea}

There is another interesting and not so widely known group of equations which can be formulated involving only the tangents of half the quantities $e_{i}, E_{I}$ and $e, E$. The excesses $\delta=2 e, \Delta=2 E$ always appear in the equations to be given below under the apparently indeterminate but finite forms $T_{\kappa_{1}}\left(\frac{e}{2}\right) / \kappa_{2}$ and $T_{\kappa_{2}}\left(\frac{E}{2}\right) / \kappa_{1}$. By taking into account the relations (4.40), these quotients can be expressed in terms of area and coarea as

$$
T_{\kappa_{1}^{2} \kappa_{2}}\left(\frac{\mathcal{S}}{4}\right)=\frac{T_{\kappa_{2}}\left(\frac{E}{2}\right)}{\kappa_{1}} \quad T_{\kappa_{1} \kappa_{2}^{2}}\left(\frac{s}{4}\right)=\frac{T_{\kappa_{1}}\left(\frac{e}{2}\right)}{\kappa_{2}}
$$

We consider now the first group of the Gauss-Delambre-Mollweide analogies (5.11) which by means of (4.34) can be written as

$$
\frac{S_{\kappa_{2}}\left(\frac{E-E_{K}}{2}\right)}{S_{\kappa_{2}}\left(\frac{E+E_{K}}{2}\right)}=-\frac{C_{\kappa_{1}}\left(\frac{e_{i}-e_{j}}{2}\right)}{C_{\kappa_{1}}\left(\frac{e_{i}+e_{j}}{2}\right)}
$$

which is equivalent to

$$
\frac{S_{\kappa_{2}}\left(\frac{E-E_{K}}{2}\right)-S_{\kappa_{2}}\left(\frac{E+E_{K}}{2}\right)}{S_{\kappa_{2}}\left(\frac{E-E_{K}}{2}\right)+S_{\kappa_{2}}\left(\frac{E+E_{K}}{2}\right)}=\frac{-C_{\kappa_{1}}\left(\frac{e_{i}-e_{j}}{2}\right)-C_{\kappa_{1}}\left(\frac{e_{i}+e_{j}}{2}\right)}{-C_{\kappa_{1}}\left(\frac{e_{i}-e_{j}}{2}\right)+C_{\kappa_{1}}\left(\frac{e_{i}+e_{j}}{2}\right)} .
$$

We apply the relations (A.12)-(A.14) and introduce tangents; thus we find a new equation:

$$
\frac{T_{\kappa_{2}}\left(\frac{E_{K}}{2}\right)}{T_{\kappa_{2}}\left(\frac{E}{2}\right)}=-\frac{1}{\kappa_{1} T_{\kappa_{1}}\left(\frac{e_{i}}{2}\right) T_{\kappa_{1}}\left(\frac{e_{j}}{2}\right)} .
$$

The remaining analogies allow similar derivations. The final set of equations coming from (5.11) is given by

$$
\begin{aligned}
& \frac{T_{\kappa_{2}}\left(\frac{E}{2}\right)}{\kappa_{1}}=-T_{\kappa_{1}}\left(\frac{e_{i}}{2}\right) T_{\kappa_{1}}\left(\frac{e_{j}}{2}\right) T_{\kappa_{2}}\left(\frac{E_{K}}{2}\right) \quad \frac{T_{\kappa_{2}}\left(\frac{E}{2}\right)}{\kappa_{1}} T_{\kappa_{2}}\left(\frac{E_{I}}{2}\right)=\frac{T_{\kappa_{1}}\left(\frac{e}{2}\right)}{\kappa_{2}} T_{\kappa_{1}}\left(\frac{e_{i}}{2}\right) \\
& \frac{T_{\kappa_{1}}\left(\frac{e_{i}}{2}\right)}{T_{\kappa_{2}}\left(\frac{E_{I}}{2}\right)}=\frac{T_{\kappa_{1}}\left(\frac{e_{j}}{2}\right)}{T_{\kappa_{2}}\left(\frac{E_{J}}{2}\right)} \frac{T_{\kappa_{1}}\left(\frac{e}{2}\right)}{\kappa_{2}}=-T_{\kappa_{2}}\left(\frac{E_{I}}{2}\right) T_{\kappa_{2}}\left(\frac{E_{J}}{2}\right) T_{\kappa_{1}}\left(\frac{e_{k}}{2}\right) .
\end{aligned}
$$

These expressions lead to a self-dual equation reminiscent to the sine theorem:

$$
\frac{T_{\kappa_{1}}\left(\frac{e_{i}}{2}\right)}{T_{\kappa_{2}}\left(\frac{E_{I}}{2}\right)}=\frac{T_{\kappa_{1}}\left(\frac{e_{j}}{2}\right)}{T_{\kappa_{2}}\left(\frac{E_{J}}{2}\right)}=\frac{T_{\kappa_{1}}\left(\frac{e_{k}}{2}\right)}{T_{\kappa_{2}}\left(\frac{E_{K}}{2}\right)}=\frac{\frac{T_{\kappa_{2}}\left(\frac{E}{2}\right)}{\kappa_{1}}}{\frac{T_{\kappa_{1}}\left(\frac{e}{2}\right)}{\kappa_{2}}}=\frac{T_{\kappa_{1}^{2} \kappa_{2}}(\mathcal{S} / 4)}{T_{\kappa_{1} \kappa_{2}^{2}}(s / 4)} .
$$

Furthermore, the above relations give rise to other expressions similar to Euler's equations:

$$
T_{\kappa_{2}}^{2}\left(\frac{E_{I}}{2}\right)=-\frac{T_{\kappa_{1} \kappa_{2}^{2}}(s / 4) T_{\kappa_{1}}\left(\frac{e_{i}}{2}\right)}{T_{\kappa_{1}}\left(\frac{e_{j}}{2}\right) T_{\kappa_{1}}\left(\frac{e_{k}}{2}\right)} \quad T_{\kappa_{1}}^{2}\left(\frac{e_{i}}{2}\right)=-\frac{T_{\kappa_{1}^{2} \kappa_{2}}(\mathcal{S} / 4) T_{\kappa_{2}}\left(\frac{E_{I}}{2}\right)}{T_{\kappa_{2}}\left(\frac{E_{J}}{2}\right) T_{\kappa_{2}}\left(\frac{E_{K}}{2}\right)}
$$

and a pair of mutually dual equations:

$$
T_{\kappa_{1}^{2} \kappa_{2}}^{2}\left(\frac{\mathcal{S}}{4}\right)=-\frac{T_{\kappa_{1}}\left(\frac{e}{2}\right)}{\kappa_{2}} T_{\kappa_{1}}\left(\frac{e_{i}}{2}\right) T_{\kappa_{1}}\left(\frac{e_{j}}{2}\right) T_{\kappa_{1}}\left(\frac{e_{k}}{2}\right)
$$




$$
T_{\kappa_{1} \kappa_{2}^{2}}^{2}\left(\frac{s}{4}\right)=-\frac{T_{\kappa_{2}}\left(\frac{E}{2}\right)}{\kappa_{1}} T_{\kappa_{2}}\left(\frac{E_{I}}{2}\right) T_{\kappa_{2}}\left(\frac{E_{J}}{2}\right) T_{\kappa_{2}}\left(\frac{E_{K}}{2}\right) .
$$

A large number of expressions for area and coarea of a triangle loop in terms of sides and angles can be easily obtained by simple analitical transformations involving the identities given in the Appendix. In what follows we deduce only some equations for area (or angular excess (4.40)), but clearly any expression can be dualized and remains true, so the reader can easily write down the dual formulas for the coarea of a triangle loop.

We first give formulas for trigonometric functions of half the area:

$$
\begin{aligned}
C_{\kappa_{1}^{2} \kappa_{2}}\left(\frac{\mathcal{S}}{2}\right)= & \frac{1+C_{\kappa_{1}}\left(x_{i}\right)+C_{\kappa_{1}}\left(x_{j}\right)+C_{\kappa_{1}}\left(x_{k}\right)}{4 C_{\kappa_{1}}\left(\frac{x_{i}}{2}\right) C_{\kappa_{1}}\left(\frac{x_{j}}{2}\right) C_{\kappa_{1}}\left(\frac{x_{k}}{2}\right)} \\
S_{\kappa_{1}^{2} \kappa_{2}}\left(\frac{\mathcal{S}}{2}\right)= & -\frac{S_{\kappa_{2}}\left(X_{I}\right) S_{\kappa_{1}}\left(x_{j}\right) S_{\kappa_{1}}\left(x_{k}\right)}{4 C_{\kappa_{1}}\left(\frac{x_{i}}{2}\right) C_{\kappa_{1}}\left(\frac{x_{j}}{2}\right) C_{\kappa_{1}}\left(\frac{x_{k}}{2}\right)}=-\frac{S_{\kappa_{2}}\left(X_{I}\right) S_{\kappa_{1}}\left(\frac{x_{j}}{2}\right) S_{\kappa_{1}}\left(\frac{x_{k}}{2}\right)}{C_{\kappa_{1}}\left(\frac{x_{i}}{2}\right)} \\
= & \frac{\left\{-\frac{S_{\kappa_{1}}(e)}{\kappa_{2}} S_{\kappa_{1}}\left(e_{i}\right) S_{\kappa_{1}}\left(e_{j}\right) S_{\kappa_{1}}\left(e_{k}\right)\right\}^{1 / 2}}{2 C_{\kappa_{1}}\left(\frac{x_{i}}{2}\right) C_{\kappa_{1}}\left(\frac{x_{j}}{2}\right) C_{\kappa_{1}}\left(\frac{x_{k}}{2}\right)} \\
T_{\kappa_{1}^{2} \kappa_{2}}\left(\frac{\mathcal{S}}{2}\right)= & -\frac{S_{\kappa_{2}}\left(X_{I}\right) S_{\kappa_{1}}\left(x_{j}\right) S_{\kappa_{1}}\left(x_{k}\right)}{1+C_{\kappa_{1}}\left(x_{i}\right)+C_{\kappa_{1}}\left(x_{j}\right)+C_{\kappa_{1}}\left(x_{k}\right)} \\
& =2 \frac{\left\{-\frac{S_{\kappa_{1}}(e)}{\kappa_{2}} S_{\kappa_{1}}\left(e_{i}\right) S_{\kappa_{1}}\left(e_{j}\right) S_{\kappa_{1}}\left(e_{k}\right)\right\}^{1 / 2}}{1+C_{\kappa_{1}}\left(x_{i}\right)+C_{\kappa_{1}}\left(x_{j}\right)+C_{\kappa_{1}}\left(x_{k}\right)} .
\end{aligned}
$$

In order to prove these equations we write

$$
\begin{aligned}
& S_{\kappa_{1}^{2} \kappa_{2}}\left(\frac{\mathcal{S}}{2}\right)=\frac{S_{\kappa_{2}}(E)}{\kappa_{1}}=\frac{1}{\kappa_{1}} S_{\kappa_{2}}\left(\frac{X_{I}}{2}+\frac{X_{J}+X_{K}}{2}\right) \\
& =\frac{1}{\kappa_{1}}\left\{S_{\kappa_{2}}\left(\frac{X_{I}}{2}\right) C_{\kappa_{2}}\left(\frac{X_{J}+X_{K}}{2}\right)+S_{\kappa_{2}}\left(\frac{X_{J}+X_{K}}{2}\right) C_{\kappa_{2}}\left(\frac{X_{I}}{2}\right)\right\} ;
\end{aligned}
$$

by introducing the analogies (5.11) and grouping common terms we find that

$$
=\frac{S_{\kappa_{2}}\left(\frac{X_{I}}{2}\right) C_{\kappa_{2}}\left(\frac{X_{I}}{2}\right)}{\kappa_{1} C_{\kappa_{1}}\left(\frac{x_{i}}{2}\right)}\left\{C_{\kappa_{1}}\left(\frac{x_{j}+x_{k}}{2}\right)-C_{\kappa_{1}}\left(\frac{x_{j}-x_{k}}{2}\right)\right\}
$$

and by applying (A.13) and (A.4) we prove the second equation of (5.23):

$$
=-2 \frac{S_{\kappa_{2}}\left(\frac{X_{I}}{2}\right) C_{\kappa_{2}}\left(\frac{X_{I}}{2}\right) S_{\kappa_{1}}\left(\frac{x_{j}}{2}\right) S_{\kappa_{1}}\left(\frac{x_{k}}{2}\right)}{C_{\kappa_{1}}\left(\frac{x_{i}}{2}\right)}=-\frac{S_{\kappa_{2}}\left(X_{I}\right) S_{\kappa_{1}}\left(\frac{x_{j}}{2}\right) S_{\kappa_{1}}\left(\frac{x_{k}}{2}\right)}{C_{\kappa_{1}}\left(\frac{x_{i}}{2}\right)} .
$$

The relation (A.4) allows us to find the first equation of (5.23), while (5.5) leads to the third one. The cosine equation (5.22) is obtained by using (A.1) and the first identity of (A.36) starting with the third equation of (5.23). By taking quotients in (5.22) and (5.23) we find (5.24). Furthermore, by introducing in the first expression of (5.24) the cosine theorem for $C_{\kappa_{1}}\left(x_{i}\right)$, and applying the relations (A.4) and (A.5), we obtain:

$$
T_{\kappa_{1}^{2} \kappa_{2}}\left(\frac{\mathcal{S}}{2}\right)=-\frac{S_{\kappa_{2}}\left(X_{I}\right) T_{\kappa_{1}}\left(\frac{x_{j}}{2}\right) T_{\kappa_{1}}\left(\frac{x_{k}}{2}\right)}{1-\kappa_{1} C_{\kappa_{2}}\left(X_{I}\right) T_{\kappa_{1}}\left(\frac{x_{j}}{2}\right) T_{\kappa_{1}}\left(\frac{x_{k}}{2}\right)} .
$$


The equation (5.22) gives rise to a relation for the cosine of $\mathcal{S}$ by applying (A.5), while (5.22) and the first equation of (5.23) give rise to a relation for the sine by means of (A.4):

$$
\begin{aligned}
C_{\kappa_{1}^{2} \kappa_{2}}(\mathcal{S}) & =\frac{\left[1+C_{\kappa_{1}}\left(x_{i}\right)+C_{\kappa_{1}}\left(x_{j}\right)+C_{\kappa_{1}}\left(x_{k}\right)\right]^{2}-8 C_{\kappa_{1}}^{2}\left(\frac{x_{i}}{2}\right) C_{\kappa_{1}}^{2}\left(\frac{x_{j}}{2}\right) C_{\kappa_{1}}^{2}\left(\frac{x_{k}}{2}\right)}{8 C_{\kappa_{1}}^{2}\left(\frac{x_{i}}{2}\right) C_{\kappa_{1}}^{2}\left(\frac{x_{j}}{2}\right) C_{\kappa_{1}}^{2}\left(\frac{x_{k}}{2}\right)} \\
S_{\kappa_{1}^{2} \kappa_{2}}(\mathcal{S}) & =-\frac{S_{\kappa_{1}}\left(x_{i}\right) S_{\kappa_{1}}\left(x_{j}\right) S_{\kappa_{2}}\left(X_{K}\right)\left[1+C_{\kappa_{1}}\left(x_{i}\right)+C_{\kappa_{1}}\left(x_{j}\right)+C_{\kappa_{1}}\left(x_{k}\right)\right]}{8 C_{\kappa_{1}}^{2}\left(\frac{x_{i}}{2}\right) C_{\kappa_{1}}^{2}\left(\frac{x_{j}}{2}\right) C_{\kappa_{1}}^{2}\left(\frac{x_{k}}{2}\right)}
\end{aligned}
$$

Finally, the relation (5.22) leads to two functions of $\mathcal{S} / 4$ by considering (A.5) and (A.35), and by making use of (A.6) and (A.34). The expressions so obtained are:

$$
\begin{aligned}
& C_{\kappa_{1}^{2} \kappa_{2}}^{2}\left(\frac{\mathcal{S}}{4}\right)=\frac{C_{\kappa_{1}}\left(\frac{e}{2}\right) C_{\kappa_{1}}\left(\frac{e_{i}}{2}\right) C_{\kappa_{1}}\left(\frac{e_{j}}{2}\right) C_{\kappa_{1}}\left(\frac{e_{k}}{2}\right)}{C_{\kappa_{1}}\left(\frac{x_{i}}{2}\right) C_{\kappa_{1}}\left(\frac{x_{j}}{2}\right) C_{\kappa_{1}}\left(\frac{x_{k}}{2}\right)} \\
& S_{\kappa_{1}^{2} \kappa_{2}}^{2}\left(\frac{\mathcal{S}}{4}\right)=-\frac{\frac{S_{\kappa_{1}}\left(\frac{e}{2}\right)}{\kappa_{2}} S_{\kappa_{1}}\left(\frac{e_{i}}{2}\right) S_{\kappa_{1}}\left(\frac{e_{j}}{2}\right) S_{\kappa_{1}}\left(\frac{e_{k}}{2}\right)}{C_{\kappa_{1}}\left(\frac{x_{i}}{2}\right) C_{\kappa_{1}}\left(\frac{x_{j}}{2}\right) C_{\kappa_{1}}\left(\frac{x_{k}}{2}\right)} .
\end{aligned}
$$

Notice that the quotient of these relations allows us to recover (5.21).

\subsection{Some historical comments}

Trigonometry, motivated mainly in the spherical case by astronomy, has a very interesting and well documented history. A good authoritative reference for its historical development is the book by Rozenfel'd [38]; Ratcliffe [35] contains also historical notes. A standard reference for spherical trigonometry covering all the spherical versions of the equations we have discussed here and much more - which suitably reformulated also extends without exception to the nine geometries - is the book by Todhunter-Leathem [39]. Hyperbolic trigonometry was first satisfactorily settled by Lobachewski and Bolyai and is an essential tool when studying hyperbolic manifolds; see e.g. [34]-[37].

The consideration of cosine and dual cosine in the forms 1i, 1I as the basic equations of spherical trigonometric dates back to Euler, but the 'alternative' form 1'i is much older and essentially is the one given by Regiomontanus, ellaborating on earlier results. The spherical cosine equations themselves are usually ascribed to Albategnius, and the first explicit appearance of the dual cosine equations is ascribed to Vieta, even though spherical polarity was clear to al-Ṭ̂̄si in the thirteenth century. The sine theorem, in another equivalent form, appears in Menelaus. The formulas (5.1)-(5.4) are the general $\left(\kappa_{1}, \kappa_{2}\right)$ versions of the spherical formulas due to Euler, while (5.11) are the general versions of Gauss-Delambre-Mollweide analogies (in the old meaning of proportion), and (5.12) are the general version of the Napier analogies. The first formula for the sine in (5.23) is known in the spherical case as Cagnoli's theorem, and the expression for the area (or excess) of a spherical triangle in terms of the sides (5.21) is due to L'Huillier, extending the euclidean Heron-Archimedes area formula. Most formulas for spherical triangle area in terms of sides and/or angles were obtained by Euler. Other formulas for the trigonometric functions of one half or one fourth the area are also known in the spherical case, but bear no name; some are due to L'Huillier and Serret. It is interesting to remark that suitably reformulated all these formulas hold for the nine CK plane geometries, and the full richness in the trigonometry of each individual space shows up at full only when studying the whole set of the nine spaces. 


\subsection{Existence conditions}

Browsing through the equations in Section 5.1, it appears clearly that the two quantities

$$
\begin{aligned}
& \left\{-S_{\kappa_{1}^{2} \kappa_{2}}(\mathcal{S} / 2) S_{\kappa_{2}}\left(E_{I}\right) S_{\kappa_{2}}\left(E_{J}\right) S_{\kappa_{2}}\left(E_{K}\right)\right\}^{1 / 2}=\left\{-\frac{S_{\kappa_{2}}(E)}{\kappa_{1}} S_{\kappa_{2}}\left(E_{I}\right) S_{\kappa_{2}}\left(E_{J}\right) S_{\kappa_{2}}\left(E_{K}\right)\right\}^{1 / 2} \\
& \left\{-S_{\kappa_{1} \kappa_{2}^{2}}(s / 2) S_{\kappa_{1}}\left(e_{i}\right) S_{\kappa_{1}}\left(e_{j}\right) S_{\kappa_{1}}\left(e_{k}\right)\right\}^{1 / 2}=\left\{-\frac{S_{\kappa_{1}}(e)}{\kappa_{2}} S_{\kappa_{1}}\left(e_{i}\right) S_{\kappa_{1}}\left(e_{j}\right) S_{\kappa_{1}}\left(e_{k}\right)\right\}^{1 / 2}
\end{aligned}
$$

must be real in order to the triangle to exist, so the quantities under the square root must be non-negative. The sides and angles of a triangle in the CK space with constants $\kappa_{1}, \kappa_{2}$ must satisfy the inequalities:

$$
-\frac{S_{\kappa_{2}}(E)}{\kappa_{1}} S_{\kappa_{2}}\left(E_{I}\right) S_{\kappa_{2}}\left(E_{J}\right) S_{\kappa_{2}}\left(E_{K}\right) \geq 0 \quad-\frac{S_{\kappa_{1}}(e)}{\kappa_{2}} S_{\kappa_{1}}\left(e_{i}\right) S_{\kappa_{1}}\left(e_{j}\right) S_{\kappa_{1}}\left(e_{k}\right) \geq 0 .
$$

In terms of the explicit notation (4.35), the relation relative to sides reads:

$$
\frac{S_{\kappa_{1}}(p-a)}{\kappa_{2}} S_{\kappa_{1}}(p) S_{\kappa_{1}}(p-b) S_{\kappa_{1}}(p-c) \geq 0
$$

and by using the identities (A.28) and (A.29), this can also be rewritten as:

$$
\frac{1}{\kappa_{2}}\left\{V_{\kappa_{1}}(b+c)-V_{\kappa_{1}}(a)\right\}\left\{V_{\kappa_{1}}(a)-V_{\kappa_{1}}(b-c)\right\} \geq 0 .
$$

This inequality must be satisfied by the sides of the triangle loop. When $\kappa_{2}=0$ we already know that $a=b+c$, so it suffices to discuss the cases $\kappa_{2} \neq 0$. When $\kappa_{2}$ is positive, both terms in brackets must have the same sign, and if $\kappa_{2}$ is negative, each term must have a different sign. A completely similar inequality holds, mutatis mutandis, for angles.

The complete discussion of the inequalities that sides and angles must satisfy can be done starting from this, but requires attention to subcases when any of the constants $\kappa_{1}$, $\kappa_{2}$ is positive. The derivative of the versed sine function $V_{\kappa}(x)$ for a generic label $\kappa$ is the sine: $\frac{d}{d x} V_{\kappa}(x)=S_{\kappa}(x)$; hence $V_{\kappa}(x)$ is increasing for all values of $x$ when the label is zero or negative, but for positive $\kappa$, when $x$ is larger than a quadrant, $x \geq \frac{\pi}{2 \sqrt{\kappa}}$ (see $(6.2)$ below), the versed sine is decreasing. If when $\kappa_{1}>0$ (resp. $\kappa_{2}>0$ ) we restrict sides (resp. angles) to less than a quadrant, and label $a$ the largest side (resp. $A$ the largest external angle) then it is easy to transform the basic inequalities for sides and angles to give:

$$
\begin{array}{ll}
B+C>A>|B-C| \quad \text { when } \quad \kappa_{1}>0 & B+C<A \text { when } \kappa_{1}<0 \\
b+c>a>|b-c| \quad \text { when } \kappa_{2}>0 & b+c<a \text { when } \kappa_{2}<0
\end{array}
$$

which reduce to the known conditions in the three riemannian cases. These inequalities can be also and more clearly expressed in terms of the (half) excesses $E$ and $e$, and read:

$$
\begin{aligned}
& E>0 \quad \text { when } \kappa_{1}>0 \quad E<0 \text { when } \kappa_{1}<0 \\
& e>0 \text { when } \kappa_{2}>0 \quad e<0 \text { when } \kappa_{2}<0 \text {. }
\end{aligned}
$$




\section{$6 \quad$ Other types of triangles}

In the general CK space $S O_{\kappa_{1}, \kappa_{2}}(3) / S O_{\kappa_{2}}(2)$ there are four types of triangles according to the kind of the sides: (i) the three sides are first-kind (time-like), (ii) two first-kind and one second-kind, (iii) one first-kind and two second-kind, and (iv) three second-kind (space-like). Of course when $\kappa_{2}>0$, first- and second-kind lines coincide so that the four types merge into one. Triangles with isotropic sides are limiting non-generic cases. In this Section we present the main equations which characterize the trigonometry of pure second-kind triangles of type (iv) and 'mixed' triangles of type (ii); for the latter we will only consider orthogonal triangles.

\subsection{Second-kind triangles}

With rather obvious modifications the method we have developed for the pure first-kind triangles of type (i) will give the equations of the pure second-kind ones of type (iv). For the latter all sides are second-kind lengths (i.e., space lengths in the kinematical cases) and the translations along sides will be conjugated to the generator $P_{2}$ instead of $P_{1}$. Henceforth, the label of sides will be the product $\kappa_{1} \kappa_{2}$ and not $\kappa_{1}$ (see (2.7) and (2.13)), which in turn means that the equations for the second-kind triangles can be directly obtained by replacing $\kappa_{1}$ by $\kappa_{1} \kappa_{2}$ in all relations for first-kind triangles. Consequently, the general appearance of these new equations is very similar to the pure first-kind ones, but now the side lengths are of second-kind. As an example we only write the cosine, dual cosine and sine theorems for a pure second-kind triangle:

$$
\begin{aligned}
& C_{\kappa_{1} \kappa_{2}}\left(x_{i}\right)=C_{\kappa_{1} \kappa_{2}}\left(x_{j}\right) C_{\kappa_{1} \kappa_{2}}\left(x_{k}\right)-\kappa_{1} \kappa_{2} S_{\kappa_{1} \kappa_{2}}\left(x_{j}\right) S_{\kappa_{1} \kappa_{2}}\left(x_{k}\right) C_{\kappa_{2}}\left(X_{I}\right) \\
& C_{\kappa_{2}}\left(X_{I}\right)=C_{\kappa_{2}}\left(X_{J}\right) C_{\kappa_{2}}\left(X_{K}\right)-\kappa_{2} S_{\kappa_{2}}\left(X_{J}\right) S_{\kappa_{2}}\left(X_{K}\right) C_{\kappa_{1} \kappa_{2}}\left(x_{i}\right) \\
& \frac{S_{\kappa_{1} \kappa_{2}}\left(x_{i}\right)}{S_{\kappa_{2}}\left(X_{I}\right)}=\frac{S_{\kappa_{1} \kappa_{2}}\left(x_{j}\right)}{S_{\kappa_{2}}\left(X_{J}\right)}=\frac{S_{\kappa_{1} \kappa_{2}}\left(x_{k}\right)}{S_{\kappa_{2}}\left(X_{K}\right)}
\end{aligned}
$$

to be compared with the first-kind equations 1i, 1I and 2 of (4.36).

For $\kappa_{2}>0$ both translation generators are conjugated, so the trigonometry for pure first-kind and second-kind triangles is essentially identical, differing only by the fact that the sides should be measured according to $P_{2}$. For $\kappa_{2}<0$ the sign in the cosine equations for sides will change, and equations for triangles with second-kind sides will be different from the first-kind ones. In the $(1+1) \mathrm{D}$ case, the difference between anti-de Sitter and de Sitter spacetimes reduces simply to the interchange between time-like and space-like

lines, so the trigonometry of pure space-like triangles in de Sitter spacetime (resp. anti-de Sitter) coincides with the trigonometry of time-like ones in anti-de Sitter spacetime (resp. de Sitter). In the $(1+1) \mathrm{D}$ minkowskian spacetime the interchange of time-like and spacelike lines is a symmetry, and again the only difference is the factor $c=1 / \sqrt{-\kappa_{2}}$ between the length of any segment measured in 'time' or in 'space' units. In our approach, this symmetry is encoded by the interchange $\kappa_{1} \leftrightarrow \kappa_{1} \kappa_{2}\left(P_{1} \leftrightarrow P_{2}\right)$ with $\kappa_{2}\left(J_{12}\right)$ invariant.

\subsection{Orthogonal triangles}

The two remaining types of triangles, (ii) and (iii), are 'mixed' triangles with sides of two different kind and are something different to the pure ones. They are important because 
triangles with two orthogonal sides belong necessarily to these types (in the kinematical cases, a direction orthogonal to a time-like direction must be space-like).

The equations of the trigonometry of such triangles could be obtained by suitably adapting the method introduced in Section 3. But there is also an alternative way, which allows us to formally derive the equations of any type of triangles from the pure first-kind ones; we will sketch this procedure. It is based in the consideration of the angle between two orthogonal directions; this is a real angle when $\kappa_{2}>0$ but is only a formal device (an ideal angle) when $\kappa_{2} \leq 0$. This orthogonal angle or quadrant of angle will be defined by

$$
\left\llcorner_{\kappa_{2}}:=\frac{\pi}{2 \sqrt{\kappa_{2}}}\right.
$$

which is well defined whenever $\kappa_{2} \neq 0$ and has a real value when $\kappa_{2}>0$; if $\kappa_{2}=0$ then $L_{\kappa_{2}}=\infty$. This verifies

$$
C_{\kappa_{2}}\left(\left\llcorner_{\kappa_{2}}\right)=0 \quad S_{\kappa_{2}}\left(\left\llcorner_{\kappa_{2}}\right)=\frac{1}{\sqrt{\kappa_{2}}}\right.\right.
$$

Of course, a quadrant of length could be analogously defined; this will be real when $\kappa_{1}>0$ as in the case of the sphere, but will be only a formal device when $\kappa_{1}<0$, e.g. for hyperbolic geometry.

We restrict here to the equations of trigonometry for orthogonal triangles with two first-kind sides $a$ and $b$ and one second-kind side $h$ orthogonal to the side $a$. This kind of triangle has an inner angle $C$ at the vertex $a \cap b$ and an 'external' angle $A$ (between the side $h$ and the line orthogonal to the side $b$ as shown in figure 5) at vertex $b \cap h$.

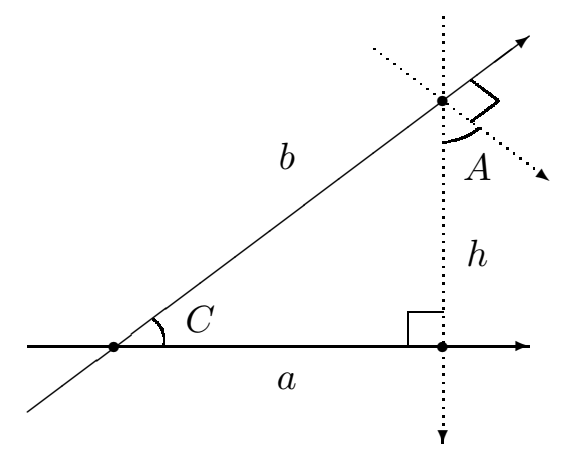

Figure 5: Orthogonal triangle with two first-kind sides $a$ and $b$, one second-kind side $h$, an inner angle $C$ and an external angle $A$.

For the generic situation with $\kappa_{2} \neq 0$, this orthogonal triangle can be obtained as a formal case of a pure first-kind one (compare figure 5 to figure 4) where out of the six quantities $a, b, c, A, B, C$ associated to a first-kind triangle $A, B$ and $c$ should be understood through the replacements $A \rightarrow A+\left\llcorner_{\kappa_{2}}, B \rightarrow L_{\kappa_{2}}\right.$ and $c$ (with label $\kappa_{1}$ ) $\rightarrow h$ (with label $\kappa_{1} \kappa_{2}$ ), while $a, b$ and $C$ are unchanged. By taking into account the properties (6.3), we find that this transformation can be described by the following substitution:

$$
\begin{aligned}
& a \rightarrow a \quad b \rightarrow b \quad C \rightarrow C \\
& C_{\kappa_{1}}(c) \rightarrow C_{\kappa_{1} \kappa_{2}}(h) \quad C_{\kappa_{2}}(A) \rightarrow-\sqrt{\kappa_{2}} S_{\kappa_{2}}(A) \quad C_{\kappa_{2}}(B) \rightarrow 0 \\
& S_{\kappa_{1}}(c) \rightarrow \sqrt{\kappa_{2}} S_{\kappa_{1} \kappa_{2}}(h) \quad S_{\kappa_{2}}(A) \rightarrow \frac{1}{\sqrt{\kappa_{2}}} C_{\kappa_{2}}(A) \quad S_{\kappa_{2}}(B) \rightarrow \frac{1}{\sqrt{\kappa_{2}}} .
\end{aligned}
$$


The trigonometric relations for the orthogonal triangle can be straightforwardly obtained from those corresponding to the first-kind one (given in Section 4) by simply applying the above transformations. Hence the theorems (4.7)-(4.9) give rise to

- Three cosine theorems for sides:

$$
\begin{aligned}
& C_{\kappa_{1}}(a)=C_{\kappa_{1}}(b) C_{\kappa_{1} \kappa_{2}}(h)+\kappa_{1} \kappa_{2} S_{\kappa_{1}}(b) S_{\kappa_{1} \kappa_{2}}(h) S_{\kappa_{2}}(A) \\
& C_{\kappa_{1}}(b)=C_{\kappa_{1}}(a) C_{\kappa_{1} \kappa_{2}}(h) \\
& C_{\kappa_{1} \kappa_{2}}(h)=C_{\kappa_{1}}(a) C_{\kappa_{1}}(b)+\kappa_{1} S_{\kappa_{1}}(a) S_{\kappa_{1}}(b) C_{\kappa_{2}}(C) .
\end{aligned}
$$

- Three cosine theorems for angles:

$$
\begin{aligned}
& S_{\kappa_{2}}(A)=S_{\kappa_{2}}(C) C_{\kappa_{1}}(a) \\
& 0=-S_{\kappa_{2}}(A) C_{\kappa_{2}}(C)+C_{\kappa_{2}}(A) S_{\kappa_{2}}(C) C_{\kappa_{1}}(b) \\
& C_{\kappa_{2}}(C)=C_{\kappa_{2}}(A) C_{\kappa_{1} \kappa_{2}}(h) .
\end{aligned}
$$

- One sine theorem:

$$
\frac{S_{\kappa_{1}}(a)}{C_{\kappa_{2}}(A)}=S_{\kappa_{1}}(b)=\frac{S_{\kappa_{1} \kappa_{2}}(h)}{S_{\kappa_{2}}(C)}
$$

We stress that although the maps (6.4) are well defined only for the generic case with $\kappa_{2} \neq 0$, in the resulting equations (6.5)-(6.7) the contraction $\kappa_{2}=0$ is always well defined. We also remark that the three relations (6.5) and the third one of (6.6) can be expressed in terms of versed sines avoiding the trivial identities $1=1$ when $\kappa_{1}$ or $\kappa_{2}$ is equal to zero. For instance, when $\kappa_{1}=0$ the second relation of (6.5) reduces simply to $b^{2}=a^{2}+\kappa_{2} h^{2}$ (the 'pythagorean' theorem for sides), while the third equation of (6.6) gives $C^{2}=A^{2}+\kappa_{1} h^{2}$ when $\kappa_{2}=0$.

Other interesting relations, which can be obtained from (6.5)-(6.7), read

$$
\begin{gathered}
C_{\kappa_{2}}(C)=\frac{T_{\kappa_{1}}(a)}{T_{\kappa_{1}}(b)} \quad T_{\kappa_{2}}(C)=\frac{T_{\kappa_{1} \kappa_{2}}(h)}{S_{\kappa_{1}}(a)} \\
S_{\kappa_{2}}(A)=\frac{T_{\kappa_{1} \kappa_{2}}(h)}{T_{\kappa_{1}}(b)} \quad T_{\kappa_{2}}(A)=\frac{S_{\kappa_{1} \kappa_{2}}(h)}{T_{\kappa_{1}}(a)} .
\end{gathered}
$$

The dependence between all these relations is described by:

Theorem 4. For an orthogonal triangle in any of the nine 2D CK geometries, there are always three independent equations. Any other trigonometric relation follows from them. When $\kappa_{1} \neq 0$ and $\kappa_{2} \neq 0$, a possible choice for these three equations is:

$$
C_{\kappa_{1}}(b)=C_{\kappa_{1}}(a) C_{\kappa_{1} \kappa_{2}}(h) \quad C_{\kappa_{2}}(C)=C_{\kappa_{2}}(A) C_{\kappa_{1} \kappa_{2}}(h) \quad S_{\kappa_{1} \kappa_{2}}(h)=S_{\kappa_{1}}(b) S_{\kappa_{2}}(C) .
$$

When $\kappa_{1}=0$ (resp. $\kappa_{2}=0$ ) the first (resp. second) equation should be replaced by $b^{2}=a^{2}+\kappa_{2} h^{2}\left(\right.$ resp. $\left.C^{2}=A^{2}+\kappa_{1} h^{2}\right)$.

The proof is a matter of simple algebraic manipulations and is omitted.

The last interesting quantity to introduce is the area; starting again from its definition for a first-kind triangle, $\mathcal{S}=(B+C-A) / \kappa_{1}$, and applying the transformation of the angles we find for the area for an orthogonal triangle:

$$
\mathcal{S}=\frac{C-A}{\kappa_{1}}
$$


Table 3: Equations of trigonometry for orthogonal triangles for the nine CK spaces.

\begin{tabular}{|c|c|c|}
\hline $\begin{array}{l}\text { Elliptic }(+1,+1) \\
\quad S O(3) / S O(2)\end{array}$ & $\begin{array}{l}\text { Euclidean }(0,+1) \\
I S O(2) / S O(2)\end{array}$ & $\begin{array}{c}\text { Hyperbolic } \quad(-1,+1) \\
S O(2,1) / S O(2)\end{array}$ \\
\hline $\begin{aligned} \cos b & =\cos a \cos h \\
\cos C & =\cos A \cos h \\
\sin h & =\sin b \sin C \\
\tan h & =\tan b \sin A \\
\sin a & =\sin b \cos A \\
\tan a & =\tan b \cos C\end{aligned}$ & $\begin{array}{c}b^{2}=a^{2}+h^{2} \\
C=A \\
h=b \sin C\end{array}$ & $\begin{aligned} \cosh b & =\cosh a \cosh h \\
\cos C & =\cos A \cosh h \\
\sinh h & =\sinh b \sin C \\
\tanh h & =\tanh b \sin A \\
\sinh a & =\sinh b \cos A \\
\tanh a & =\tanh b \cos C\end{aligned}$ \\
\hline $\sin \mathcal{S}=\frac{\sin a \sin h}{1+\cos b}$ & $\mathcal{S}=\frac{1}{2} a h$ & $\sin \mathcal{S}=\frac{\sinh a \sinh h}{1+\cosh b}$ \\
\hline $\begin{array}{cc}\text { Co-Euclidean } & (+1,0) \\
\text { Oscillating NH } & I S O(2) / \mathbb{R}\end{array}$ & $\begin{array}{c}\text { Galilean }(0,0) \\
I I S O(1) / \mathbb{R}\end{array}$ & $\begin{array}{l}\text { Co-Minkowskian }(-1,0) \\
\text { Expanding NH } I S O(1,1) / \mathbb{R}\end{array}$ \\
\hline $\begin{array}{c}b=a \\
C^{2}=A^{2}+h^{2} \\
h=C \sin b \\
h=A \tan b\end{array}$ & $\begin{array}{c}b=a \\
C=A \\
h=b C\end{array}$ & $\begin{array}{c}b=a \\
C^{2}=A^{2}-h^{2} \\
h=C \sinh b \\
h=A \tanh b\end{array}$ \\
\hline $\mathcal{S}=\frac{h \sin a}{1+\cos b}$ & $\mathcal{S}=\frac{1}{2} a h$ & $\mathcal{S}=\frac{h \sinh a}{1+\cosh b}$ \\
\hline $\begin{array}{c}\text { Co-Hyperbolic }(+1,-1) \\
\text { Anti-de Sitter } S O(2,1) / S O(1,1)\end{array}$ & $\begin{array}{l}\text { Minkowskian }(0,-1) \\
\quad I S O(1,1) / S O(1,1)\end{array}$ & $\begin{array}{c}\text { Doubly Hyperbolic }(-1,-1) \\
\text { De Sitter } S O(2,1) / S O(1,1)\end{array}$ \\
\hline $\begin{array}{c}\cos b=\cos a \cosh h \\
\cosh C=\cosh A \cosh h \\
\sinh h=\sin b \sinh C \\
\tanh h=\tan b \sinh A \\
\sin a=\sin b \cosh A \\
\tan a=\tan b \cosh C\end{array}$ & $\begin{array}{c}b^{2}=a^{2}-h^{2} \\
C=A \\
h=b \sinh C\end{array}$ & $\begin{array}{c}\cosh b=\cosh a \cos h \\
\cosh C=\cosh A \cos h \\
\sin h=\sinh b \sinh C \\
\tan h=\tanh b \sinh A \\
\sinh a=\sinh b \cosh A \\
\tanh a=\tanh b \cosh C\end{array}$ \\
\hline $\sinh \mathcal{S}=\frac{\sin a \sinh h}{1+\cos b}$ & $\mathcal{S}=\frac{1}{2} a h$ & $\sinh \mathcal{S}=\frac{\sinh a \sin h}{1+\cosh b}$ \\
\hline
\end{tabular}

An expression for the sine of the area can be easily deduced:

$$
\begin{aligned}
& S_{\kappa_{1}^{2} \kappa_{2}}(\mathcal{S})=\frac{1}{\kappa_{1}} S_{\kappa_{2}}(C-A)=\frac{1}{\kappa_{1}}\left(S_{\kappa_{2}}(C) C_{\kappa_{2}}(A)-S_{\kappa_{2}}(A) C_{\kappa_{2}}(C)\right) \\
& =\frac{S_{\kappa_{1}}(a) S_{\kappa_{1} \kappa_{2}}(h)}{\kappa_{1} S_{\kappa_{1}}^{2}(b)}\left(1-\frac{C_{\kappa_{1}}^{2}(b)}{C_{\kappa_{1} \kappa_{2}}(h) C_{\kappa_{1}}(a)}\right)=\frac{S_{\kappa_{1}}(a) S_{\kappa_{1} \kappa_{2}}(h)}{1-C_{\kappa_{1}}^{2}(b)}\left(1-C_{\kappa_{1}}(b)\right)
\end{aligned}
$$

which finally gives

$$
S_{\kappa_{1}^{2} \kappa_{2}}(\mathcal{S})=\frac{S_{\kappa_{1}}(a) S_{\kappa_{1} \kappa_{2}}(h)}{1+C_{\kappa_{1}}(b)}
$$

When $\kappa_{1}=0$, we recover the flat space familiar value $\mathcal{S}=\frac{1}{2} a h$. In the same way we find

$$
C_{\kappa_{1}^{2} \kappa_{2}}(\mathcal{S})=\frac{C_{\kappa_{1}}(a)+C_{\kappa_{1} \kappa_{2}}(h)}{1+C_{\kappa_{1}}(b)}
$$

We show in table 3 the main trigonometric equations and the area relation (6.12) for these orthogonal triangles particularised in each of the nine 2D CK spaces to the standard values of $\kappa_{i}$ equal to $+1,0$ and -1 ; the first three equations are independent. Note that self-duality does not hold for orthogonal triangles; the dual of an orthogonal triangle would be a triangle with a side whose length equals to a quadrant. 
Table 4: Some equations involving the area $\mathcal{S}$ and coarea $s$ of a time-like triangle in the six homogeneous spacetimes with (time) radius $\tau$ (curvature $\kappa_{1}= \pm 1 / \tau^{2}$ ) and relativistic constant $c\left(\kappa_{2}=-1 / c^{2}\right)$. Here the three sides are the (proper) time intervals $\tau_{a}, \tau_{b}, \tau_{c}$ and $\tau_{p}:=\left(\tau_{a}+\tau_{b}+\tau_{c}\right) / 2$. The angles are the relative rapidities $\chi_{A}, \chi_{B}, \chi_{C}$ and $\chi_{P}:=$ $\left(\chi_{A}+\chi_{B}+\chi_{C}\right) / 2$.

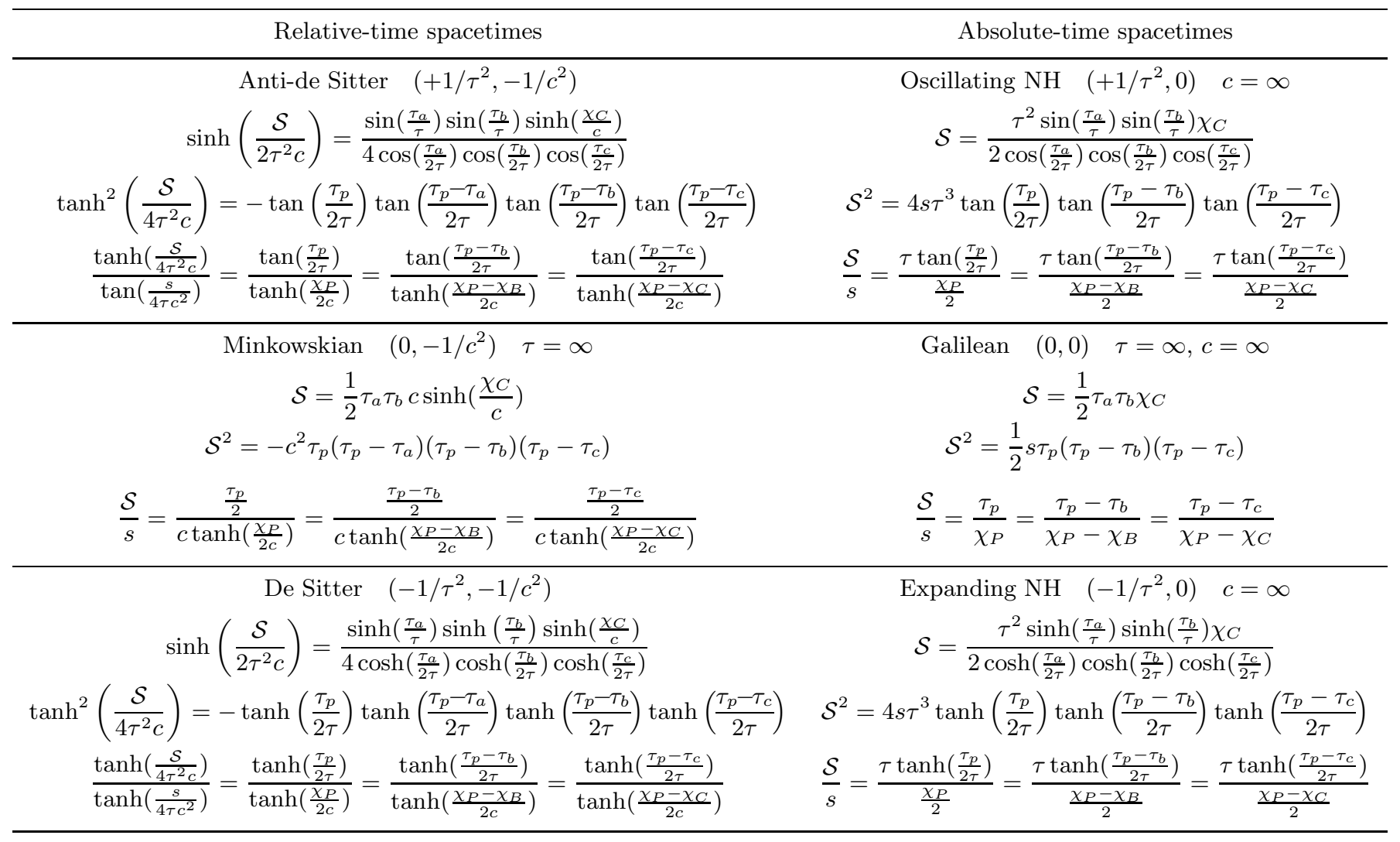

\section{$7 \quad$ On the trigonometry of homogeneous spacetimes}

Once familiarity is gained with the labelled cosine, sine and tangent functions, the general way of writing the equations of trigonometry becomes clearer than the conventional formulation. However in order to facilitate the reading of these general equations, we present in table 4 a sample of equations for the area in the six homogeneous spacetimes: the generalised formula of Cagnoli (first equation in (5.23)) and that of Heron-L'Huillier (5.21) as well as the equation (5.19) that relates area and coarea. We use the conventional units, and keep explicitly the universe (time) radius $\tau$ and the relativistic constant $c$; recall that the CK constants in the kinematical interpretation (2.9) of the CK space of points as $(1+1) \mathrm{D}$ spacetime are $\kappa_{1}= \pm 1 / \tau^{2}$ and $\kappa_{2}=-1 / c^{2}$ (see Section 2.3).

To avoid confusion of the relativistic constant $c$ with a side, and also to stress that these sides are proper times, we will denote in this Section the (time) side lengths by $\tau_{a}, \tau_{b}, \tau_{c}$, while angles in the $(1+1) \mathrm{D}$ spacetime are kinematically rapidities, and will be denoted according to the traditional notation as $\chi_{A}, \chi_{B}, \chi_{C}$. Should these equations be extended to include the $\kappa_{2}>0$ case (say let $\kappa_{2}=1 / c^{2}$ ), the $c$ would play the role of a conversion constant between radians and the chosen angular measure. 
A suitable view of the general trigonometric equations is as a kind of deformation of the purely linear equations (4.22). The deformation is governed by two constants $\kappa_{1}, \kappa_{2}$, whose geometrical role is that of spacetime curvatures and/or signatures of the metric. From this viewpoint, it is clear that the good 'totally flat' reference $2 \mathrm{D}$ geometry is not the euclidean one, but should be instead the galilean geometry, whose trigonometric equations are purely linear; all others being better described by its departures, governed by $\kappa_{1}, \kappa_{2}$, relative to the galilean one. The kinematical interpretation of the three basic trigonometric equations in galilean spacetime, where rapidities, defined as the canonical parameter of subgroups of pure inertial transformations, are equal to ordinary velocities, is clear:

$$
\tau_{a}=\tau_{b}+\tau_{c} \quad \chi_{A}=\chi_{B}+\chi_{C} \quad \frac{\tau_{a}}{\chi_{A}}=\frac{\tau_{b}}{\chi_{B}}=\frac{\tau_{c}}{\chi_{C}} .
$$

The first equation means that the (proper) time interval along any future time-like curve depends only on the endpoints, and corresponds to the absolute time; the same equation holds in both Newton-Hooke cases (see table 2). The second is the additivity, in galilean spacetime, of the relative rapidities of three non-concurrent free motions; this also holds in the minkowskian case, but not in the four curved spacetimes. This relation should not be confused with the additivity of relative rapidities for coplanar and concurrent free motions, which holds in all cases because rapidities are defined as canonical parameters of the one-parameter subgroup generated by $\mathcal{K}$. The third equation states that relative rapidities and time interval lengths in any triangle in galilean spacetime are proportional; this is an absolutely elementary property of classical spacetime and holds only in this case. For the area and coarea of a galilean triangle we have:

$$
\mathcal{S}=\frac{1}{2} \chi_{A} \tau_{b} \tau_{c} \quad s=\frac{1}{2} \tau_{a} \chi_{B} \chi_{C} .
$$

We recall that coarea of a triangular loop in the six kinematical spaces is (proportional) to the difference of actions for a free particle following either of the two worldlines $C B$ and $C A B$ which determine the triangle loop [40,41].

These purely linear equations allow a deformation in two different senses [17], either by endowing spacetime with curvature $\kappa_{1} \neq 0$ (obtaining the two Newton-Hooke spacetimes), or keeping it flat but introducing curvature, necessarily negative if causality must be preserved, in the space of time-like lines, described by the constant $\kappa_{2}<0$ (obtaining the minkowskian spacetime of special relativity). If both processes are simultaneously made, we obtain the two de Sitter spacetimes.

This structural 'unfolding' of the complete CK scheme starting from its most degenerate case runs in a striking parallel with the historical development. Spacetime is nearly flat at the time and length human scales: this fact lies behind classical physics. With the present hindsight, we can say that to assume a flat (i.e., $\kappa_{1}=0$ ) homogeneous model for the $(1+1) \mathrm{D}$ spacetime involved in $1 \mathrm{D}$ kinematics was natural. At the human speed (or even solar system) scale, the curvature in the space of uniform motions is also negligible, so to assume again a flat space of motions (or time-like lines) - embodied in the equality $\kappa_{2}=0$ for the $1 \mathrm{D}$ kinematic group-, was the only practical choice. Both assumptions greatly simplified (or rather, allowed) the linear mathematical description of classical physics. However, even at the homogeneous level of approximation, Nature does not seem to be characterized by these non-generic choices. Relativity can be described as the discovery of a negative curvature in the space of $1 \mathrm{D}$ motions, and then the allimportant relativistic constant $c$ appears simply as related to the value of the curvature 
of this space of motions by $\kappa_{2}=-1 / c^{2}$. Special relativity still keeps a flat spacetime, another approximation which is abandoned, in a way much more general than by assuming it homogeneous, in the context of general relativity; if homogeneity is still kept, the possibilities are $\kappa_{1}= \pm 1 / \tau^{2}$.

Before spacetime geometry was under consideration, a similar situation happened for the physical 3D space geometry, whose characterization among the mathematical possibilities was at the root of Riemann's program. General 3D CK spaces are parametrized by three CK constants, say $\mu_{1}, \mu_{2}, \mu_{3}$ and correspond to a space with constant curvature $\mu_{1}$ and whose metric is reducible at each point to $\operatorname{diag}\left(1, \mu_{2}, \mu_{2} \mu_{3}\right)$; restriction to a locally euclidean space is embodied into the choices $\mu_{2}>0, \mu_{3}>0$. In the $(1+3) \mathrm{D}$ homogeneous spacetime $S O_{\kappa_{1}, \kappa_{2}, \kappa_{3}, \kappa_{4}}(5) / S O_{\kappa_{2}, \kappa_{3}, \kappa_{4}}(4)$ (see Section 2.1), whose labels are $\kappa_{1}= \pm 1 / \tau^{2}$, $\kappa_{2}=-1 / c^{2}, \kappa_{3}=1, \kappa_{4}=1$, the three-space orthogonal to the fiducial time-like line through the origin (and hence all three-spaces orthogonal to any time-like line) can be identified with the homogenous space $S O_{\kappa_{1} \kappa_{2}, \kappa_{3}, \kappa_{4}}(4) / S O_{\kappa_{3}, \kappa_{4}}(3)$. Hence the constants $\mu_{1}, \mu_{2}, \mu_{3}$ are given by $\mu_{1}=\kappa_{1} \kappa_{2}, \mu_{2}=\kappa_{3}, \mu_{3}=\kappa_{4}$ so that the curvature of the threespace orthogonal to a given time-like direction is the product $\kappa_{1} \kappa_{2}=\mp 1 /\left(\tau^{2} c^{2}\right)$. This means that in the non-relativistic spacetimes, even if spacetime is curved, the three-space is flat. In the three relativistic cases, three-space is only flat in the minkowskian case, but is curved in the anti-de Sitter (space curvature $-1 /\left(\tau^{2} c^{2}\right)$ ) and de Sitter spacetimes (space curvature $\left.1 /\left(\tau^{2} c^{2}\right)\right)$. In both cases the universe radius $R$, with dimensions of space-length, is given by $R=c \tau$.

Here we have also a clear example of hidden universal constants, in the sense given to this term by Lévy-Leblond [42]: both $\kappa_{3}$ and $\kappa_{4}$ are not usually considered as universal constants only because the fact that they are non-zero and positive allow to make them apparently dissapear by reducing them to the value 1 (thus making plane angles and dihedral space angles apparently dimensionless). Once performed, this reduction forbids further consideration of these constants and the possibility they could be either zero or negative in other conceivable but still homogeneous spaces is simply out of sight. The character of $\kappa_{1} \kappa_{2}$ as a possible universal constant was understood much earlier: Lobachewski explored the possibility of physical three-space geometry being hyperbolic (i.e. negatively curved), and tried to give experimental bounds to a constant he called $k$ (in modern terms, the curvature would be $-1 / k^{2}$ ) for our physical space under the assumption, later disproven by Einstein's theory, that light travels along geodesics in this physical three-space; the argument is based on the existence of a minimum parallax (for a given baseline) even for infinitely distant stars [7, 43]. The founding fathers of hyperbolic geometry could have hardly imagined that the geometry they were discovering/inventing was indeed realized by Nature and to a good approximation, not as the geometry of space itself, but as the geometry of the space of uniform motions.

The equations we have developed can be used to discuss many questions. For instance, a recent paper by Jing-Ling Chen and Mo-Lin Ge [44] identifies the Wigner angle of the rotation appearing in the product of pure Lorentz transformations to the defect of a triangle in hyperbolic geometry; this and similar results follow also directly from our approach, because in the $(1+3) \mathrm{D}$ spacetime labeled by $\kappa_{1}, \kappa_{2}=-1 / c^{2}, \kappa_{3}=1, \kappa_{4}=1$ the geometry of the 3D set of time-like lines through a fixed spacetime point is characterized by the three last labels $\kappa_{2}, \kappa_{3}, \kappa_{4}$, so it is $\left\{-1 / c^{2}, 1,1\right\}$. This geometry is hyperbolic in the three relativistic cases degenerating to euclidean in the three non-relativistic ones. Similar results involving triangle defects appear also in relation to geometrical phases. Triangles 
in anti-de Sitter and de Sitter spaces also display properties similar to the 'parallelism angle' found in hyperbolic geometry, and are related to the existence of horizons.

\section{Concluding remarks}

As far as we know, the approach we have given to trigonometry of the real CK spaces (the symmetric rank-one homogeneous spaces of real type and their limiting spaces) is new. Furthermore and even in this case of rank-one spaces of real type, we have also obtained some results apparently unknown on the trigonometry of several spacetimes.

In spite of these remarks, the value of any new method for studying such a venerable body of knowledge as trigonometry could be considered with some scepticism, especially if this method were only applicable to the family of spaces we have discussed in this paper. This is definitely not the case and we feel that the main value of this paper is to display in this simplest case the potentialities this group-theoretical approach to trigonometry has for the study of many other interesting spaces whose trigonometry is still unknown.

Along this line, the trigonometry of complex, quaternionic and octonionic type CK spaces will be discussed in a companion forthcoming paper [21]; only the complex case is really relevant, as the others reduce directly to the complex case. The CK family of complex spaces has as their 'elliptic' member $\kappa_{1}>0, \kappa_{2}>0$ the hermitian elliptic space, which coincides with the complex projective space $\mathbb{C} P^{2}$. Therefore the mathematical structure underlying the quantum state space appears as a particular space in the complex CK family. Thus, and although primarily mathematical, the study of trigonometry in hermitian complex spaces has a very direct and deep connection with physics, the link being the geometry of the quantum space of states. Unlike the real spherical or hyperbolic trigonometry, this 'hermitian' trigonometry is not widely known yet, though several sets of basic equations in the elliptic and hyperbolic cases are established [3], [45]-[50]. The method we have developed here affords a new overall view to hermitian trigonometry $[51,52]$ and leads to a description of triangles by sides, angles, lateral phases and angular phases, which exhibits explicitly the duality in the whole family and leads to self-duality in the 'elliptic' case. This self-duality is completely hidden in the equations derived by $[3,48,49,50]$, mainly due to their choice for the basic trigonometric quantities.

These new equations can be taken as a starting point allowing the complete exploration of the still rather unknown whole zoo of hermitian trigonometric equations, at a very modest price. The essential resemblance between the method for the real and complex spaces makes the exploration of the new territory easier by consciously exploiting the analogies, while at the same time pointing out the differences with the familiar real trigonometry.

The next natural objective is the study of trigonometry of higher rank grassmannians, either real, complex or quaternionic. This is still largely unknown in the general case (see however [53]). We hope the method outlined in this paper should be able to produce in a direct form the equations of trigonometry for grassmannians, thus making a further step towards a general approach to trigonometry of any symmetric homogeneous space.

This goal will require first to group all symmetric homogeneous spaces into CK families, and then to study trigonometry for each family. Work on this line is also in progress [20], and opens the possibility of realizing all simple Lie algebras, even the special linear ones, $S O^{*}(2 n), S U^{*}(2 n)$ and the exceptional ones, as 'unitary' algebras, leaving invariant 
an 'hermitian' (relative to some antiinvolution) form over a tensor product of two pseudodivision algebras. This realization should allow a quick checking on whether or not some extension of the ideas outlined here afford the equations of trigonometry for any homogeneous space in an explicit and simple enough way. In this area only very few results are known; for instance, the trigonometry of the rank-two spaces $S U(3)$ and $S L(3, \mathbb{C}) / S U(3)$ is discussed by $[5,6]$, and these rely heavily on the use of the Weyl theorems on invariant theory and characterization of invariants by means of traces of products of matrices. A purely group theoretical derivation would be interesting.

\section{Acknowledgments}

This work was partially supported by DGICYT, Spain (Project PB94/1115) and Junta de Castilla y León, Spain (Project CO2/399).

\section{Appendix: Some relations for the trigonometric functions}

The main identities for the trigonometric functions defined in (2.14)-(2.16) depending on a (curvature) label $\kappa$ and involving one or two arbitrary arguments $x, y$ are given by [10]:

$$
\begin{gathered}
C_{\kappa}^{2}(x)+\kappa S_{\kappa}^{2}(x)=1 \\
C_{\kappa}(x)=1-\kappa V_{\kappa}(x) \\
C_{\kappa}(2 x)=C_{\kappa}^{2}(x)-\kappa S_{\kappa}^{2}(x) \\
S_{\kappa}(2 x)=2 S_{\kappa}(x) C_{\kappa}(x) \\
C_{\kappa}^{2}\left(\frac{x}{2}\right)=\frac{C_{\kappa}(x)+1}{2} \\
S_{\kappa}^{2}\left(\frac{x}{2}\right)=\frac{1-C_{\kappa}(x)}{2 \kappa}=\frac{1}{2} V_{\kappa}(x) \\
T_{\kappa}\left(\frac{x}{2}\right)=\frac{1-C_{\kappa}(x)}{\kappa S_{\kappa}(x)}=\frac{S_{\kappa}(x)}{C_{\kappa}(x)+1} \\
C_{\kappa}(x \pm y)=C_{\kappa}(x) C_{\kappa}(y) \mp \kappa S_{\kappa}(y) S_{\kappa}(x) \\
S_{\kappa}(x \pm y)=S_{\kappa}(x) C_{\kappa}(y) \pm S_{\kappa}(y) C_{\kappa}(x) \\
V_{\kappa}(x \pm y)=V_{\kappa}(x)+V_{\kappa}(y)-\kappa V_{\kappa}(x) V_{\kappa}(y) \pm S_{\kappa}(x) S_{\kappa}(y) \\
T_{\kappa}(x \pm y)=\frac{T_{\kappa}(x) \pm T_{\kappa}(y)}{1 \mp \kappa T_{\kappa}(x) T_{\kappa}(y)} \\
C_{\kappa}(x)+C_{\kappa}(y)=2 C_{\kappa}\left(\frac{x+y}{2}\right) C_{\kappa}\left(\frac{x-y}{2}\right) \\
C_{\kappa}(x)-C_{\kappa}(y)=-2 \kappa S_{\kappa}\left(\frac{x+y}{2}\right) S_{\kappa}\left(\frac{x-y}{2}\right) \\
S_{\kappa}(x) \pm S_{\kappa}(y)=2 S_{\kappa}\left(\frac{x \pm y}{2}\right) C_{\kappa}\left(\frac{x \mp y}{2}\right) \\
V_{\kappa}(y)=2\left\{V_{\kappa}\left(\frac{x+y}{2}\right)+V_{\kappa}\left(\frac{x-y}{2}\right)\right.
\end{gathered}
$$




$$
\begin{array}{r}
\left.-\kappa V_{\kappa}\left(\frac{x+y}{2}\right) V_{\kappa}\left(\frac{x-y}{2}\right)\right\} \\
V_{\kappa}(x)-V_{\kappa}(y)=2 S_{\kappa}\left(\frac{x+y}{2}\right) S_{\kappa}\left(\frac{x-y}{2}\right) .
\end{array}
$$

Let $x, y, z$ three arbitrary real numbers. We consider their half sum $p$ and some related quantities defined by:

$$
p=\frac{x+y+z}{2} \quad p-x=\frac{y+z-x}{2} \quad p-y=\frac{x+z-y}{2} \quad p-z=\frac{x+y-z}{2} .
$$

Then we find the following identities involving three arbitrary arguments:

$$
\begin{aligned}
& C_{\kappa}(x+y)+C_{\kappa}(z)=2 C_{\kappa}(p) C_{\kappa}(p-z) \\
& C_{\kappa}(x-y)+C_{\kappa}(z)=2 C_{\kappa}(p-x) C_{\kappa}(p-y) \\
& C_{\kappa}(x+y)-C_{\kappa}(z)=-2 \kappa S_{\kappa}(p) S_{\kappa}(p-z) \\
& C_{\kappa}(x-y)-C_{\kappa}(z)=2 \kappa S_{\kappa}(p-x) S_{\kappa}(p-y) \\
& S_{\kappa}(x+y)+S_{\kappa}(z)=2 S_{\kappa}(p) C_{\kappa}(p-z) \\
& S_{\kappa}(x-y)+S_{\kappa}(z)=2 S_{\kappa}(p-y) C_{\kappa}(p-x) \\
& S_{\kappa}(x+y)-S_{\kappa}(z)=2 S_{\kappa}(p-z) C_{\kappa}(p) \\
& S_{\kappa}(x-y)-S_{\kappa}(z)=-2 S_{\kappa}(p-x) C_{\kappa}(p-y) \\
& V_{\kappa}(x+y)+V_{\kappa}(z)=2\left\{V_{\kappa}(p)+V_{\kappa}(p-z)-\kappa V_{\kappa}(p) V_{\kappa}(p-z)\right\} \\
& V_{\kappa}(x-y)+V_{\kappa}(z)=2\left\{V_{\kappa}(p-x)+V_{\kappa}(p-y)-\kappa V_{\kappa}(p-x) V_{\kappa}(p-y)\right\} \\
& V_{\kappa}(x+y)-V_{\kappa}(z)=2 S_{\kappa}(p) S_{\kappa}(p-z) \\
& V_{\kappa}(x-y)-V_{\kappa}(z)=-2 S_{\kappa}(p-x) S_{\kappa}(p-y) \\
& C_{\kappa}(x) S_{\kappa}(y)=C_{\kappa}(p) S_{\kappa}(p-z)+S_{\kappa}(p-x) C_{\kappa}(p-y) \\
& C_{\kappa}(x) S_{\kappa}(y)=S_{\kappa}(p) C_{\kappa}(p-z)-C_{\kappa}(p-x) S_{\kappa}(p-y) \\
& S_{\kappa}(x) S_{\kappa}(y)=S_{\kappa}(p) S_{\kappa}(p-z)+S_{\kappa}(p-x) S_{\kappa}(p-y) \\
& \kappa S_{\kappa}(x) S_{\kappa}(y)=-C_{\kappa}(p) C_{\kappa}(p-z)+C_{\kappa}(p-x) C_{\kappa}(p-y) \\
& 4 C_{\kappa}\left(\frac{x}{2}\right) C_{\kappa}\left(\frac{y}{2}\right) C_{\kappa}\left(\frac{z}{2}\right)-\left[1+C_{\kappa}(x)+C_{\kappa}(y)+C_{\kappa}(z)\right] \\
& =8 \kappa^{2} S_{\kappa}\left(\frac{p}{2}\right) S_{\kappa}\left(\frac{p-x}{2}\right) S_{\kappa}\left(\frac{p-y}{2}\right) S_{\kappa}\left(\frac{p-z}{2}\right) \\
& 4 C_{\kappa}\left(\frac{x}{2}\right) C_{\kappa}\left(\frac{y}{2}\right) C_{\kappa}\left(\frac{z}{2}\right)+\left[1+C_{\kappa}(x)+C_{\kappa}(y)+C_{\kappa}(z)\right] \\
& =8 C_{\kappa}\left(\frac{p}{2}\right) C_{\kappa}\left(\frac{p-x}{2}\right) C_{\kappa}\left(\frac{p-y}{2}\right) C_{\kappa}\left(\frac{p-z}{2}\right) \\
& 4 \kappa^{2} S_{\kappa}(p) S_{\kappa}(p-x) S_{\kappa}(p-y) S_{\kappa}(p-z) \\
& =16 C_{\kappa}^{2}\left(\frac{x}{2}\right) C_{\kappa}^{2}\left(\frac{y}{2}\right) C_{\kappa}^{2}\left(\frac{z}{2}\right)-\left[1+C_{\kappa}(x)+C_{\kappa}(y)+C_{\kappa}(z)\right]^{2} \\
& =1-C_{\kappa}^{2}(x)-C_{\kappa}^{2}(y)-C_{\kappa}^{2}(z)+2 C_{\kappa}(x) C_{\kappa}(y) C_{\kappa}(z) \\
& 4 C_{\kappa}(p) C_{\kappa}(p-x) C_{\kappa}(p-y) C_{\kappa}(p-z) \\
& =-1+C_{\kappa}^{2}(x)+C_{\kappa}^{2}(y)+C_{\kappa}^{2}(z)+2 C_{\kappa}(x) C_{\kappa}(y) C_{\kappa}(z) .
\end{aligned}
$$




\section{References}

[1] Birman G S and Nomizu K 1984 Amer. Math. Month. 91 543-549

[2] Yaglom I M 1979 A simple non-euclidean geometry and its physical basis (New York: Springer)

[3] Hsiang W-Y 1989 Ann. Global Anal. Geom. 7 29-45

[4] Leuzinger 1992 Coment. Math. Helv. 67 252-292

[5] Aslaksen H 1990 Trans. of the Amer. Math. Soc. 317 127-142

[6] Aslaksen H and Huynh H-L 1997 Laws of trigonometry in Symmetric Spaces, Geometry from the Pacific Rim, ed A J Berrick et al (Berlin: Walter de Gruyter) 23-26

Aslaksen $\mathrm{H}$ and Huynh H-L Laws of trigonometry in $S U(3)$ and $S L(3, \mathbb{C}) / S U(3)$, Preprint, Nat. Univ. Singapore

[7] Bonola 1955 Non-euclidean geometry (New York: Dover)

[8] Martin G E 1986 The foundations of geometry and the non-euclidean plane (New York: Springer)

[9] Ballesteros A, Herranz F J, del Olmo M A and Santander M 1993 J. Phys. A: Math. Gen. 26 5801-5823

[10] Herranz F J 1995 Grupos de Cayley-Klein clásicos y cuánticos PhD Thesis University of Valladolid

[11] Ballesteros A, Herranz F J, del Olmo M A and Santander M 1995 J. Math. Phys. 36 $631-640$

[12] Santander M and Herranz F J 1997 Int. J. Mod. Phys. A 12 99-107

[13] Santander M and Herranz F J 1997 A Cayley-Klein scheme for all quasisimple real Lie algebras and Freudenthal magic squares Physical Applications and Mathematical Aspects of Geometry, Groups and Algebras vol 1, ed H-D Doebner et al (Singapore: World Scientific) 151-156

[14] Herranz F J and Santander M 1997 J. Phys. A: Math. Gen. 30 5411-5426

[15] de Azcárraga J A, Herranz F J, Pérez Bueno J C and Santander M 1998 J. Phys. A: Math. Gen. 31 1373-1394

[16] Herranz F J and Santander M 1998 Homogeneous phase spaces: the Cayley-Klein framework Geometría y Física vol XXXII, ed J F Cariñena et al (Madrid: Real Academia de Ciencias) 59-84

[17] Herranz F J and Santander M 1999 J. Phys. A: Math. Gen. 32 3743-3754

[18] Rañada M F and Santander M 1999 J. Math. Phys. 40 5026-5057

[19] Santander M 1999 Symmetric homogeneous spaces in Classical and Quantum Mechanics: a viewpoint for classification Symmetries in Quantum Mechanics and Quantum Optics, ed A Ballesteros et al (Burgos: University of Burgos) 335-352 
[20] Santander M Division algebras and the classifications of simple Lie algebras and of symmetric homogeneous spaces. In preparation

[21] Ortega R and Santander M Trigonometry of the Quantum Space of States and Hermitian Trigonometry. In preparation

[22] Wang H C 1952 Ann. Math. 55 172-191

[23] Helgason S 1978 Differential geometry, Lie groups and symmetric spaces (New York: Academic Press)

[24] Arnol'd V I 1995 Uspekhi Mat. Nauk 50:1 3-68; English transl. in Russian Math. Surveys 50:1 1-68

[25] Baxter R J 1983 Comm. Math. Phys. 88185

[26] Zamolodchikov A B 1981 Comm. Math. Phys. 79489

[27] 1989 Yang-Baxter equation in integrable systems ed M Jimbo Advanced series in Math. and Phys. vol 10 (Singapore: World Scientific)

[28] Sommerville D M Y 1910 Proc. Edinburgh Math. Soc. 28 25-41

[29] Yaglom I M, Rozenfel'd B A and Yasinskaya E U 1966 Sov. Math. Surveys 1949

[30] Kobayashi S and Nomizu K 1963 Foundations of differential geometry Vol. 1 (New York: Wiley-Interscience)

[31] Inönü E and Wigner E P 1953 Proc. Natl. Acad. Sci. USA 39510

Inönü E and Wigner E P 1954 Proc. Natl. Acad. Sci. USA 40119

[32] Bacry H and Lévy-Leblond J M 1968 J. Math. Phys. 91605

[33] Lévy-Leblond J M and Provost J P 1979 Am. J. Phys 47 1045-1049

Lévy-Leblond J M 1979 Am. J. Phys 48 345-347

[34] Berger M 1987 Geometry I, II (Berlin: Springer)

[35] Ratcliffe J G 1994 Foundations of hyperbolic manifolds (New York: Springer)

[36] Ramsay A and Richtmyer R D 1995 Introduction to hyperbolic geometry (New York: Springer)

[37] Alekseevskij D V, Vinberg E B and Solodonikov A S 1993 Geometry of spaces of constant curvature, Encyclopaedia of Mathematical Sciences vol 29 (Berlin: Springer)

[38] Rozenfel'd B A 1988 A history of non-euclidean geometry (New York: Springer)

[39] Todhunter I (rev. Leathem J G) 1901 Spherical Trigonometry (London: Macmillan)

[40] del Olmo M A and Santander M 1989 J. Phys. A: Math. Gen. 22 L763

[41] del Olmo M A and Santander M 1990 J. Geom. Phys 7171

[42] Lévy-Leblond J M 1977 Riv. Nouv. Cimento 7 187-214 
[43] Milnor J 1982 Bull Am Math Soc 69

[44] Jing-Ling Chen, Mo-Lin Ge 1998 J. Geom. Phys 25 341-345

[45] Coolidge J L 1921 Ann. of Math. 22 11-28

[46] Blaschke W and Terheggen H 1939 Rend. Sem. Mat. Univ. Roma 4(3) 153-161

[47] Terheggen H 1940 Jareshber Deutsche Math. Verein. 50 24-35

[48] Sirokov P 1957 Mat. Sb. (N. S.) 41(83) 361-372 (In russian) (1959 Math. Rews. 20 $2755 \mathrm{a}, \mathrm{b})$

Rozenfel'd 1957 Mat. Sb. (N. S.) 41(83) 373-380 (In russian) (1959 Math. Rews. 20 $2756 \mathrm{a}, \mathrm{b})$

[49] Brehm U 1990 Geom. Dedicata 33 59-76

[50] Rozenfel'd B A 1997 Geometry of Lie groups (Dordrecht: Kluwer)

[51] Ortega R and Santander M 1998 Geometry and Physics, ed M Asorey and J F Cariñena, Anales de Física Monografías 5, (Madrid: Ciemat and RSEF), p 209

[52] Ortega R and Santander M 1999 Geometrical phases and trigonometry of the quantum space of states Symmetries in Quantum Mechanics and Quantum Optics, ed A Ballesteros et al (Burgos: University of Burgos) 223-233

[53] Hangan T 1992 Rend. Sem. Mat. Univ. Pol. Torino 50 367-380 